\title{
WestVirginiaUniversity
}

THE RESEARCH REPOSITORY @ WVU

Graduate Theses, Dissertations, and Problem Reports

2013

\section{Aerodynamic Parameter Identification of a Venus Lander}

Robert A. Sykes

West Virginia University

Follow this and additional works at: https://researchrepository.wvu.edu/etd

\section{Recommended Citation}

Sykes, Robert A., "Aerodynamic Parameter Identification of a Venus Lander" (2013). Graduate Theses, Dissertations, and Problem Reports. 612.

https://researchrepository.wvu.edu/etd/612

This Thesis is protected by copyright and/or related rights. It has been brought to you by the The Research Repository @ WVU with permission from the rights-holder(s). You are free to use this Thesis in any way that is permitted by the copyright and related rights legislation that applies to your use. For other uses you must obtain permission from the rights-holder(s) directly, unless additional rights are indicated by a Creative Commons license in the record and/ or on the work itself. This Thesis has been accepted for inclusion in WVU Graduate Theses, Dissertations, and Problem Reports collection by an authorized administrator of The Research Repository @ WVU. For more information, please contact researchrepository@mail.wvu.edu. 


\title{
Aerodynamic Parameter Identification of a Venus Lander
}

\author{
Robert A. Sykes \\ Thesis submitted to the \\ Benjamin M. Statler College of Engineering and Mineral Resources \\ at West Virginia University \\ in partial fulfillment of the requirements \\ for the degree of \\ Master of Science \\ in \\ Aerospace Engineering \\ John Loth, Ph.D., Chair \\ Juan R. Cruz, Ph.D. \\ Mark Schoenenberger, M.S. \\ Shrikanth Gururajan, Ph.D. \\ Department of Mechanical and Aerospace Engineering
}

Morgantown, West Virginia

2013

Keywords: Aerodynamic System Identification, Aerodynamic Parameter Identification,

Venus Lander, Aerodynamic Coefficients, Flight Vehicle Stability

Copyright 2013 Robert A. Sykes 


\title{
Abstract \\ Aerodynamic Parameter Identification of a Venus Lander
}

\author{
Robert A. Sykes
}

An analysis was conducted to identify the parameters of an aerodynamic model for a Venus lander based on experimental free-flight data. The experimental free-flight data were collected in the NASA Langley 20-ft Vertical Spin Tunnel with a 25-percent Froude-scaled model. The experimental data were classified based on the wind tunnel run type: runs where the lander model was unperturbed over the course of the run, and runs were the model was perturbed (principally in pitch, yaw, and roll) by the wind tunnel operator. The perturbations allow for data to be obtained at higher wind angles and rotation rates than those available from the unperturbed data. The model properties and equations of motion were used to determine experimental values for the aerodynamic coefficients. An aerodynamic model was selected using a priori knowledge of axisymmetric blunt entry vehicles. The least squares method was used to estimate the aerodynamic parameters. Three sets of results were obtained from the following data sets: perturbed, unperturbed, and the combination of both. The combined data set was selected for the final set of aerodynamic parameters based on the quality of the results. The identified aerodynamic parameters are consistent with that of the static wind tunnel data. Reconstructions, of experimental data not used in the parameter identification analyses, achieved similar residuals as those with data used to identify the parameters. Simulations of the experimental data, using the identified parameters, indicate that the aerodynamic model used is incapable of replicating the limit cycle oscillations with stochastic peak amplitudes observed during the test. 


\section{Acknowledgements}

I am grateful to all of the members of my advising committee. Without their guidance this thesis would not have been possible. I am especially thankful for the tutorship and time of Dr. Juan R. Cruz.

I wish to acknowledge NASA for awarding me a Graduate Student Research Project Fellowship. The financial assistance and the opportunity to work with NASA engineers has been truly a blessing. Thanks to Mark Schoenenberger for arranging the fellowship award at NASA and to Dr. John Loth and Pam Gelet for the funding arrangements at West Virginia University.

The Atmospheric Flight and Entry Systems Branch at NASA Langley has my gratitude for supporting me during my time in residence on center.

For helping me get my foot in the door at NASA, I wish to thank Walt Engelund.

Thank you to my friends and family for their support. I am especially grateful to my fiancé Ashley Levelle for her love and support. 


\section{Table of Contents}

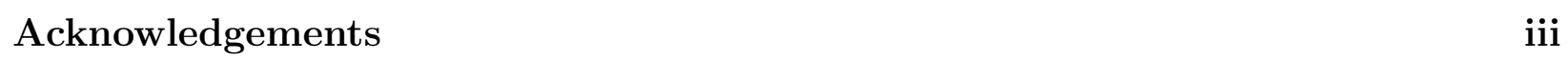

Table of Contents $\quad$ iv

List of Tables $\quad$ vii

List of Figures viii

List of Symbols $\quad$ x

List of Acronyms $\quad$ xiv

\begin{tabular}{lll}
\hline & Introduction & 1
\end{tabular}

1.1 Motivation and Objectives . . . . . . . . . . . . . . . . . . . 1

$1.2 \quad$ Venus Surface and Atmosphere Geochemical Explorer . . . . . . . . . . . . . 2

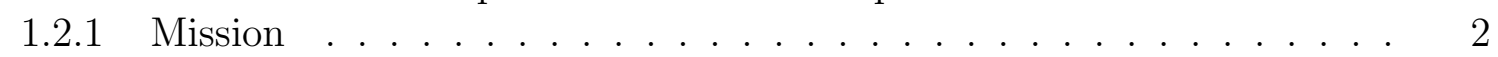

1.2 .2 Entry, Descent and Landing Sequence . . . . . . . . . . . . . . . . . . 2

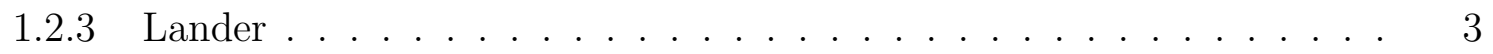

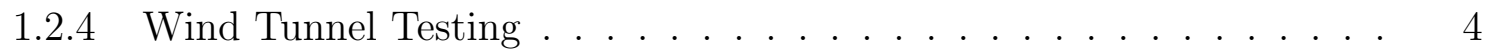

1.3 Thesis Outline . . . . . . . . . . . . . . . . . . . . . . . . . . . . . . . . 4

2 Wind Tunnel Testing $\quad 6$

2.1 Dynamic Wind Tunnel Testing . . . . . . . . . . . . . . . . . 6

2.1 .1 Test Facility . . . . . . . . . . . . . . . . . . . . . . . . . . . . 6

2.1 .2 Lander Scale Model . . . . . . . . . . . . . . . . . . . . . . . . . . 7

2.1 .3 Test Description . . . . . . . . . . . . . . . . . . . . . . . . . . . . . . . . . . . .

2.1 .4 Data Acquired . . . . . . . . . . . . . . . . . . . . . . . . . . . . . . . . . . . . . . . . . . . . .

2.1 .5 Test Results . . . . . . . . . . . . . . . . . . . . . . . . . . . 11

2.2 Static Wind Tunnel Testing . . . . . . . . . . . . . . . . . . . . . . . . . 12

2.2 .1 Test Facility . . . . . . . . . . . . . . . . . . . . . . . . . . . . . . . . 12

2.2 .2 Lander Scale Model . . . . . . . . . . . . . . . . . . . . . . . . . . . . 12

2.2 .3 Test Description $\quad \ldots \ldots \ldots \ldots \ldots$

2.2 .4 Data Acquired . . . . . . . . . . . . . . . . . . . . . . . 12 


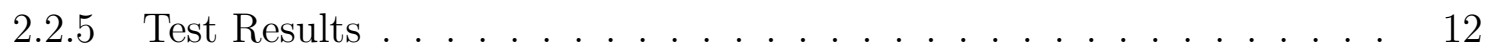

$\begin{array}{llr}3 & \text { Data Preprocessing } & 18\end{array}$

3.1 Global Smoothing Routine . . . . . . . . . . . . . . . . . . . . 18

$3.1 .1 \quad$ Fourier Reconstruction $\ldots \ldots \ldots \ldots$

3.1 .2 Filter Design and Implementation . . . . . . . . . . . . . . . . . 20

3.1 .3 Cutoff Frequencies . . . . . . . . . . . . . . . . . . . . . 21

3.2 Smoothed Derivatives . . . . . . . . . . . . . . . . . . . . . . . . . 21

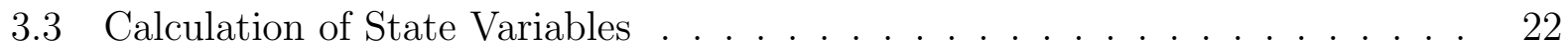

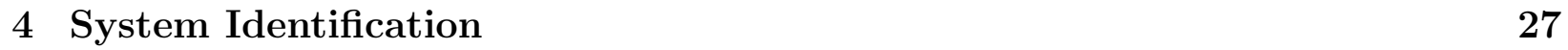

$4.1 \quad$ Aerodynamic Modeling . . . . . . . . . . . . . . . . . . . . . . . . 27

4.1 .1 Model Structure Determination . . . . . . . . . . . . . . . . 27

$4.1 .2 \quad$ Aerodynamic Model $\ldots \ldots \ldots$

4.2 Parameter Identification $\ldots \ldots \ldots a n$

4.2 .1 Ordinary Least Squares Estimator. . . . . . . . . . . . . . . . 31

$4.2 .2 \quad$ Accuracy Estimates $\ldots \ldots \ldots \ldots \ldots . \ldots \ldots$

5 Results 33

5.1 Source Data . . . . . . . . . . . . . . . . . . . . . . . . . . . . 33

$5.2 \quad$ PID Results $\ldots \ldots \ldots \ldots \ldots$

5.2 .1 Unperturbed Results . . . . . . . . . . . . . . . . . . . . 35

5.2 .2 Perturbed Results . . . . . . . . . . . . . . . . . . . . . . . . 36

5.2 .3 Combined Results. . . . . . . . . . . . . . . . . . . . . . . . . . 38

5.2 .4 Selected Parameters $\ldots \ldots \ldots$

5.3 Validation . . . . . . . . . . . . . . . . . . . . . . . . 40

5.3 .1 Static Comparison . . . . . . . . . . . . . . . . . . 40

5.3 .2 Predictive Comparison . . . . . . . . . . . . . . . . . . . . . . 40

5.3 .3 Simulation Comparison $\ldots \ldots \ldots \ldots$. . . . . . . . . . . . . 41

6 Conclusions and Future Work 53

6.1 Conclusions . . . . . . . . . . . . . . . . . . . . . 53

6.2 Recommendations for Future Work $\ldots \ldots \ldots$. . . . . . . . . 54

\begin{tabular}{ll}
\hline References & 56
\end{tabular}

\begin{tabular}{ll}
\hline A Wind Tunnel Test Data & 57
\end{tabular}

A.1 Static Data . . . . . . . . . . . . . . . . . . . . . . . . 57

A.2 Dynamic Data . . . . . . . . . . . . . . . . . . . . . . . . . . . . . . . . . 58

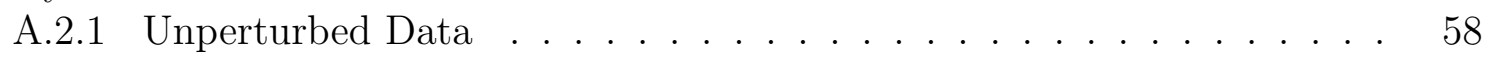

A.2.2 $\quad$ Perturbed Data . . . . . . . . . . . . . . . . . . . . . . . . . . . . . . . 59

A.3 Noise Content of the Dynamic Data . . . . . . . . . . . . . . . . . 62 
\begin{tabular}{|lr}
\hline B Flight Mechanics Equations & $\mathbf{6 6}$
\end{tabular}

B.1 Introduction . . . . . . . . . . . . . . . . . . . . . 66

B.2 Assumptions . . . . . . . . . . . . . . . . . . . . . . . . . 66

B.3 Rigid Body Equations of Motion . . . . . . . . . . . . . . . . . . . 66

B.4 Gravitational Forces . . . . . . . . . . . . . . . . . . . . . . . 67

B.5 Aerodynamic Forces and Moments . . . . . . . . . . . . . . . 68

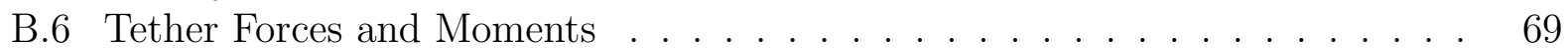

B.6.1 Force Magnitude . . . . . . . . . . . . . . . . . . . . . . . . . . . . 69

B.6.2 Tether Geometry . . . . . . . . . . . . . . . . . . . . . . 70

B.7 Auxiliary Equations . . . . . . . . . . . . . . . . . . . . . . . . . . . 70

B.7.1 Airspeed and Wind Components . . . . . . . . . . . . . 70

B.7.2 Dynamic Pressure and Wind Angles . . . . . . . . . . . . . . 72

B.7.3 Additional Equations $\ldots \ldots \ldots \ldots \ldots$

B.8 Shifting the Static Aerodynamic Moment

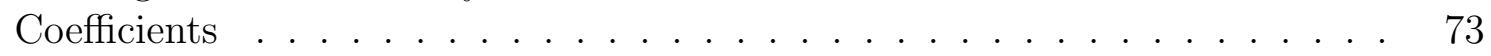

B.9 Transformation of Coordinate Systems and

Euler Angles . . . . . . . . . . . . . . . . . . . . . . . . . 73

\begin{tabular}{lr}
\hline C Code Verification & $\mathbf{7 6}$
\end{tabular}

C.1 Simulated Data . . . . . . . . . . . . . . . . . . . . . . . . . . 76

C.2 Equations of Motion . . . . . . . . . . . . . . . . . . . . 76

C.3 Parameter Identification of Simulated Data . . . . . . . . . . . . . . 76 


\section{List of Tables}

$2.1 \quad$ NASA LaRC VST specifications . . . . . . . . . . . . . . . . . . . . . . . . . 7

2.2 SAGE full-scale and model landers $\ldots \ldots \ldots$. . . . . . . . . 8

2.3 Tether force results $\ldots \ldots \ldots \ldots \ldots$

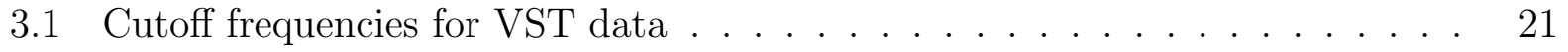

5.1 Wind tunnel run breakdown . . . . . . . . . . . . . . . . . . . . . . 34

5.2 Unperturbed PID results $\ldots \ldots \ldots \ldots \ldots$

5.3 Perturbed PID results $\ldots \ldots \ldots \ldots \ldots$

5.4 Combined PID results $\ldots \ldots \ldots \ldots$

5.5 Selected aerodynamic parameter set $\ldots \ldots \ldots \ldots \ldots$

5.6 Comparison of test data against simulation results . . . . . . . . . . . . 41

B.1 Tether force model parameters . . . . . . . . . . . . . . . . . . . . . . . . 69

C.1 Results of PID analysis on simulated data $\ldots \ldots \ldots \ldots 77$ 


\section{List of Figures}

1.1 SAGE EDL sequence $\ldots \ldots \ldots \ldots \ldots \ldots \ldots$

1.2 SAGE lander with major components labeled $\ldots \ldots \ldots \ldots$

2.1 NASA LaRC 20 -ft VST $\ldots \ldots \ldots \ldots$

2.2 SAGE lander scale model for dynamic wind tunnel test . . . . . . . . . . . . 13

2.3 Tether force testing weights $\ldots \ldots \ldots \ldots \ldots \ldots$

2.4 The geometric model-fixed coordinate system . . . . . . . . . . . . . . 14

2.5 Unperturbed data from Test Block 2 Run $2 \ldots \ldots \ldots$

2.6 Perturbed data from Test Block 3 Run $2 \ldots \ldots \ldots$

2.7 SAGE lander scale model for static wind tunnel test . . . . . . . . . . . . 17

2.8 Static wind tunnel test results $\ldots \ldots \ldots \ldots \ldots$

3.1 Fourier series construction . . . . . . . . . . . . . . . . . . . . . 24

3.2 Frequency spectrum and filter shape $\ldots \ldots \ldots \ldots \ldots \ldots$

3.3 Comparison of raw and smoothed data $\ldots \ldots \ldots \ldots$

5.1 Unperturbed results $\ldots \ldots \ldots \ldots$

5.2 Perturbed results . . . . . . . . . . . . . . . . . . . . . . . . . . . . . 45

5.3 Combined results $\ldots \ldots \ldots \ldots . \ldots \ldots \ldots$

5.4 PID results compared to the static wind tunnel test results . . . . . . . . . 49

5.5 Pitch and yaw damping function $\ldots \ldots \ldots \ldots \ldots \ldots$

5.6 Reconstruction of wind tunnel data not used in PID analysis . . . . . . . . . 50

5.7 Definition of the off-vertical angle, $\Theta \ldots \ldots \ldots \ldots \ldots . \ldots \ldots$

5.8 Comparison of test data against simulation results $\ldots \ldots \ldots \ldots$

A.1 Static wind tunnel test data. . . . . . . . . . . . . . . . . . . . 57

A.2 Raw unperturbed wind tunnel data . . . . . . . . . . . . . . . . . . 58

A.3 Raw wind tunnel data in perturbation 1 . . . . . . . . . . . . . . . . . 59

A.4 Raw wind tunnel data in perturbation $2 \ldots \ldots$. . . . . . . . . 60

A.5 Raw wind tunnel data in perturbation 3 . . . . . . . . . . . . . 61

A.6 Typical noise content of wind velocity data . . . . . . . . . . . . . . 62

A.7 Typical noise content of position data $\ldots \ldots \ldots \ldots$. . . . . . . . 63

A.8 $\quad$ Typical noise content of pitch and yaw data $\ldots \ldots \ldots$. . . . . . . . . 64

A.9 Typical noise content of roll data $\ldots \ldots \ldots \ldots$. . . . . . . . . . 65 
B.1 Tether geometry within VST . . . . . . . . . . . . . . . . . . 75

C.1 Simulated data for validation of the PID code . . . . . . . . . . . . . . . 78

C.2 Comparison of simulated and reconstructed dynamic values . . . . . . . . . . 79

C.3 Comparison of simulated and reconstructed force and moment coefficients. . 80 


\section{List of Symbols}

$b_{k}$

C

$\hat{\mathrm{C}}$

$\mathbf{C}_{\text {exp }}$

$C_{A}$

$C_{A_{T, 0}}$

$\left(C_{D} S\right)_{t r}$

$C_{l}, C_{m}, C_{n}$

$C_{l_{o}}, C_{m_{o}}, C_{n_{o}}$

$C_{l_{o, T}}$

$C_{m_{o, T}}$

$C_{m_{q+\dot{\alpha}}}, C_{n_{r-\dot{\beta}}}$

$C_{m_{\sin \alpha_{T}}}, C_{m_{\sin ^{3} \alpha_{T}}}$

$C_{m_{1}}, C_{m_{2}}$

$C_{N}$

$C_{N_{\sin \alpha_{T}}}, C_{N_{\sin ^{3} \alpha_{T}}}$

$C_{N_{T}}$

$C_{n_{1}}, C_{n_{2}}$

$C_{X}, C_{Y}, C_{Z}$

$d$

$d_{o r}$

E

F

$\mathbf{F}_{A}$

$F_{A, x}, F_{A, y}, F_{A, z}$

$\mathbf{F}_{g}$

$F_{g, x}, F_{g, y}, F_{g, z}$

$\mathbf{F}_{t r}$

$F_{t r}$
Magnitude of $k$ th term in the Fourier series expansion

Modeled aerodynamic coefficient time history vector

Estimate of the modeled aerodynamic coefficient time history vector, $\mathbf{C}$

Experimental aerodynamic coefficient time history vector

Axial aerodynamic force coefficient, $-C_{X}$

Aerodynamic parameter in the $C_{A}$ coefficient model

Tether drag area

Aerodynamic moment coefficients at the center of mass about the $x, y$, and $z$ axes, respectively

Aerodynamic moment coefficients at the center of the pressure vessel about the $x, y$, and $z$ axes, respectively

Static aerodynamic roll moment parameter

Instantaneous total static pitch and yaw moment coefficient

Damping coefficients in the pitch and yaw planes, respectively

Aerodynamic parameters in the $C_{m_{o, T}}$ coefficient model

Aerodynamic parameters in the pitch damping coefficient

Normal aerodynamic force coefficient, $-C_{Z}$

Aerodynamic parameters in the $C_{N_{T}}$ coefficient model

Total normal aerodynamic force coefficient, $\sqrt{C_{Y}^{2}+C_{Z}^{2}}$

Aerodynamic parameters in the yaw damping coefficient

Aerodynamic force coefficients in the $x, y$, and $z$ directions

Aerodynamic reference length (diameter of pressure vessel)

Outrigger diameter

Event in the timeline of the EDL sequence

Inertial force vector

Aerodynamic force vector

Aerodynamic force components

Gravity force vector

Gravity force components

Tether force vector

Tether force magnitude 


\begin{tabular}{|c|c|}
\hline$F_{t r, x}, F_{t r, y}, F_{t r, z}$ & Tether force components \\
\hline$F_{x}, F_{y}, F_{z}$ & Inertial force components \\
\hline$f$ & Frequency \\
\hline$f_{k}$ & Frequency of the $k$ th term in the Fourier series expansion \\
\hline$f_{o}$ & Cutoff frequency \\
\hline$g$ & Acceleration of gravity \\
\hline$h$ & Altitude \\
\hline$I_{x x}, I_{y y}, I_{z z}$ & Mass moments of inertia \\
\hline$I_{x y}, I_{x z}, I_{y z}$ & Mass products of inertia \\
\hline $\mathrm{i}, \mathbf{j}, \mathbf{k}$ & Unit vector components \\
\hline$i, k$ & Index \\
\hline$J$ & Objective function \\
\hline M & Inertial moment vector \\
\hline $\mathbf{M}_{A}$ & Aerodynamic moment vector \\
\hline $\mathbf{M}_{A, o}$ & Aerodynamic moment vector at center of the pressure vessel \\
\hline$M_{A, x}, M_{A, y}, M_{A, z}$ & $\begin{array}{l}\text { Aerodynamic moment components about the } x, y \text {, and } z \text { axes, } \\
\text { respectively }\end{array}$ \\
\hline$M_{A, x_{o}}, M_{A, y_{o}}, M_{A, z_{o}}$ & $\begin{array}{l}\text { Aerodynamic moment components at the center of the pressure } \\
\text { vessel about the } x, y, \text { and } z \text { axes, respectively }\end{array}$ \\
\hline $\mathbf{M}_{t r}$ & Tether moment vector \\
\hline$M_{t r, x}, M_{t r, y}, M_{t r, z}$ & $\begin{array}{l}\text { Tether moment components about the } x, y \text {, and } z \text { axes, } \\
\text { respectively }\end{array}$ \\
\hline$M_{x}, M_{y}, M_{z}$ & $\begin{array}{l}\text { Inertial moment components about the } x, y \text {, and } z \text { axes, } \\
\text { respectively }\end{array}$ \\
\hline$M_{\infty}$ & Mach number of vehicle \\
\hline$m$ & Mass of lander model used in dynamic wind tunnel testing \\
\hline$N$ & Number of data points \\
\hline$n_{p}$ & Number of parameters \\
\hline p & Unknown parameter vector of size $1 \times n_{p}$ \\
\hline$\hat{\mathbf{p}}$ & Estimate of the parameter vector, $\mathbf{p}$ \\
\hline$p, q, r$ & $\begin{array}{l}\text { Rotation rate vector components about the } x, y \text {, and } z \text { axes, } \\
\text { respectively }\end{array}$ \\
\hline$p_{s}$ & Static pressure \\
\hline$q_{W}$ & Dynamic pressure of airflow in the wind tunnel \\
\hline$q_{\infty}$ & Dynamic pressure encountered by the vehicle \\
\hline $\mathbf{R}$ & Regressor matrix of size $n_{p} \times N$ \\
\hline$R^{2}$ & Coefficient of determination \\
\hline$R_{x}, R_{y}, R_{z}$ & Euler angles used for VST data \\
\hline$R e_{\infty}$ & Reynolds number relative to the aerodynamic reference length, $d$ \\
\hline$R S E$ & Relative standard error \\
\hline $\mathbf{r}_{c m}$ & $\begin{array}{l}\text { Position vector from the center of the pressure vessel to the } \\
\text { lander's center of mass location }\end{array}$ \\
\hline
\end{tabular}




\begin{tabular}{|c|c|}
\hline $\mathbf{r}_{t}$ & $\begin{array}{l}\text { Position vector from the lander's center of mass to the tether } \\
\text { attachment point }\end{array}$ \\
\hline $\mathbf{r}_{t r}$ & $\begin{array}{l}\text { Position vector from the lander's tether attachment point to the } \\
\text { tether centering ring }\end{array}$ \\
\hline$S$ & Aerodynamic reference area, $\pi d^{2} / 4$ \\
\hline$s$ & Standard error of an estimated parameter \\
\hline$s_{\nu}^{2}$ & Noise variance \\
\hline$\left[T^{B E F}\right]$ & $\begin{array}{l}\text { Transformation matrix to transform from body to tunnel-fixed } \\
\text { coordinate systems }\end{array}$ \\
\hline$T_{k}$ & Period of $k$ th term in the Fourier series expansion \\
\hline$T_{s}$ & Static temperature \\
\hline$\left[T_{1}\right]$ & $\begin{array}{l}\text { Rotational transformation matrix to transform from tunnel-fixed } \\
\text { to body coordinates }\end{array}$ \\
\hline$t$ & Time \\
\hline $\mathbf{U}$ & Velocity vector of the lander's center of mass \\
\hline $\mathbf{U}_{\mathbf{o}}$ & Velocity vector at the center of the lander's pressure vessel \\
\hline$u, v, w$ & $\begin{array}{l}\text { Velocity vector components of the lander's center of mass along } \\
\text { the } x, y \text {, and } z \text { axes, respectively }\end{array}$ \\
\hline$u_{o}, v_{o}, w_{o}$ & $\begin{array}{l}\text { Velocity vector components at the center of the lander's pressure } \\
\text { vessel along the } x, y \text {, and } z \text { axes, respectively }\end{array}$ \\
\hline$u_{W}, v_{W}, w_{W}$ & $\begin{array}{l}\text { Wind velocity vector components at the lander's center of mass } \\
\text { along the } x, y \text {, and } z \text { axes, respectively }\end{array}$ \\
\hline$u_{\infty}, v_{\infty}, w_{\infty}$ & $\begin{array}{l}\text { Airspeed components at the lander's center of mass along the } x, \\
y, \text { and } z \text { axes, respectively }\end{array}$ \\
\hline$V_{W}$ & Wind velocity magnitude \\
\hline$V_{\infty}$ & Airspeed at the lander's center of mass \\
\hline$W_{t r}$ & Effective tether weight \\
\hline$X, Y, Z$ & Axes of the tunnel fixed coordinate system \\
\hline$X_{r}, Y_{r}, Z_{r}$ & Location of the tether centering ring in tunnel coordinates \\
\hline$X_{\text {sim }}, Y_{\text {sim }}, Z_{\text {sim }}$ & $\begin{array}{l}\text { Standard aerospace coordinate system axes used in simulation, } \\
\text { planet-fixed, North-East-Down orientation }\end{array}$ \\
\hline$x, y, z$ & Axes of the body fixed coordinate systems \\
\hline$x_{c m}, y_{c m}, z_{c m}$ & Components of center of mass location vector $\mathbf{r}_{c m}$ \\
\hline$x_{t}, y_{t}, z_{t}$ & Components of vector $\mathbf{r}_{t}$ \\
\hline$x_{t, o}, y_{t, o}, z_{t, o}$ & Components of tether attachment point vector \\
\hline$x_{t r}, y_{t r}, z_{t r}$ & Components of vector $\mathbf{r}_{t r}$ \\
\hline \multicolumn{2}{|c|}{ Angle of attack } \\
\hline Total & Total angle of attack \\
\hline Angle & Angle of sideslip \\
\hline $\begin{array}{l}\text { Ratio } \\
\text { series }\end{array}$ & $\begin{array}{l}\text { Ratio of cutoff frequency to the frequency of the } k \text { th term in the Fourier } \\
\text { series expansion }\end{array}$ \\
\hline
\end{tabular}




$\begin{array}{ll}\Theta & \text { Off-vertical angle } \\ \nu & \text { Signal noise } \\ \xi & \text { Periodic Fourier function } \\ \sigma & \text { Standard error of the residual of an aerodynamic coefficient reconstruction } \\ \Phi_{k} & \text { Filter value of the } k \text { th term in the Fourier series expansion } \\ \phi, \theta, \psi & \text { Standard aerospace Euler angles } \\ \phi_{\alpha_{T}} & \text { Total angle of attack clock angle } \\ \omega & \text { Rotation rate vector } \\ \omega_{k} & \text { Angular rate of the } k \text { th term in the Fourier series expansion } \\ \omega_{q, r} & \text { Pitch and yaw rotation rate, } \sqrt{q^{2}+r^{2}}\end{array}$




\title{
List of Acronyms
}

\author{
ARC Ames Research Center \\ EDL Entry, Descent, and Landing \\ FML Fluid Mechanics Laboratory \\ LaRC Langley Research Center \\ NASA National Aeronautics and Space Administration \\ PID Parameter Identification \\ SAGE Surface and Atmosphere Geochemical Explorer \\ SIDPAC System IDentification Programs for AirCraft \\ VST Vertical Spin Tunnel
}




\section{Chapter 1: Introduction}

\subsection{Motivation and Objectives}

\section{Motivation}

Atmospheric entry vehicles, such as aeroshells and landers, often enter the subsonic flight regime as a part of the entry, descent and landing (EDL) sequence. Such vehicles are sometimes tested in free flight to observe their subsonic dynamic behavior. The NASA Langley 20-ft Vertical Spin Tunnel (VST) provides a controlled environment for free flight testing of atmospheric entry vehicles scale models in the subsonic flight regime. The VST's airflow opposes gravity allowing the vehicle to enter a terminal descent, free fall orientation. The instrumentation housed in the VST collects data that allows for a complete characterization of the state of the vehicle. See section 2.1.1 for more information on the VST. The scale models are typically Froude-scaled such that the dynamic behavior can be directly related to the full-scale vehicle in their operating environment. See section 2.1.2 for more information on Froude scaling.

The Surface and Atmosphere Geochemical Explorer (SAGE) Venus lander was tested free flight in the VST using a Froude-scaled model. See the next section for a more detailed description the SAGE mission and lander. In the initial analysis of the VST data collected on the SAGE lander only the motion of the lander was analyzed without regard for the underlying aerodynamics. This was done to meet mission proposal needs. NASA had a later interest in analyzing the test data further, to determine the static and dynamic aerodynamic coefficients of the lander. To do this a parameter identification (PID) analysis needed to be conducted. The code was to be assembled using new and existing components to create a complete, beginning-to-end analysis that: smoothed the raw data, calculated the state and additional required variables, and identified the aerodynamic parameters of the lander. The analysis was to be general enough that it could be applied to other vehicles tested in the VST. The motivation of this research was to meet this need and provide NASA this PID capability for spin-tunnel test data. 


\section{Objectives}

The research required for this thesis was driven by the following objectives:

- Integrate, verify, and validate a PID code, assembled using existing and new components, to identify aerodynamic parameters of descent vehicles based on data collected in the VST. The code should be capable of identifying all aerodynamic parameters present in the modeled aerodynamic coefficients.

- Identify the aerodynamic parameters for the SAGE Venus lander as an exercise of the code.

- Evaluate the resulting aerodynamic parameters by comparing the results in three ways. The identified static parameters are compared to static wind tunnel tests conducted on the SAGE lander. Reconstructions of the coefficients, of dynamic data not used in the PID analyses, are compared to the experimental values. Finally, flight simulations, using the identified parameters, are compared to experimental data.

\subsection{Venus Surface and Atmosphere Geochemical Ex- plorer}

\subsubsection{Mission}

SAGE is a proposed mission to Venus which is intended to study the atmosphere, climate and surface of the planet [1]. The mission consists of a carrier, which acts as a communication link, and a lander. The lander will collect atmospheric and meteorological data during the descent stage and upon landing collect scientific information on the geology and chemistry of the planetary surface. The EDL sequence is expected to take approximately one hour and the lander will survive on the planetary surface for around three hours.

\subsubsection{Entry, Descent and Landing Sequence}

The lander is initially packaged in an aeroshell. Figure 1.1 shows an artists concept of the landing sequence. The expected EDL sequence ${ }^{*}$ is as follows:

Hypersonic Entry The EDL sequence begins $E=0$ seconds when the aeroshell enters the atmosphere. At this point the aeroshell is at an altitude of approximately $140 \mathrm{~km}$, and traveling at about $11,500 \mathrm{~m} / \mathrm{s}$.

Parachute Deployments At $E=136$ seconds the first of two parachutes deploys. A pilot parachute is initially deployed; this removes the backshell which triggers the

*Expected EDL sequence of events information obtained from mission proposal documents which are currently unpublished. 
deployment of the main parachute. At this point the vehicle is at an altitude of approximately $65 \mathrm{~km}$ and descending at approximately $165 \mathrm{~m} / \mathrm{s}$.

Heat Shield Separation At $E=151$ seconds the heat shield is separated from the lander exposing it to the atmosphere. At this point the lander is at an altitude of approximately $63 \mathrm{~km}$ and descending at a rate of approximately $46 \mathrm{~m} / \mathrm{s}$.

Outrigger Deployment At $E=181$ seconds the outriggers are deployed from the initial stowed position. At this point the lander is at an altitude of approximately $62 \mathrm{~km}$ and descending at a rate of approximately $35 \mathrm{~m} / \mathrm{s}$.

Main Parachute Release At $E=301$ seconds the lander is released from the main parachute and begins an uncontrolled terminal descent free-fall to the surface of the planet. The release occurs when the lander is at an altitude of approximately $58 \mathrm{~km}$ and descending at approximately $26 \mathrm{~m} / \mathrm{s}$.

Landing The lander is expected to impact the surface of the planet at $E=3414$ seconds. The impact velocity is to be $<10 \mathrm{~m} / \mathrm{s}$.

The terminal descent free-fall phase between the main parachute release and the landing is an important part of the EDL sequence. This phase takes the most time, around 50 minutes, and is where $58 \mathrm{~km}$ of the total $140 \mathrm{~km}$ altitude for the descent is lost. This is partly because the Venusian atmosphere is much denser than earth's at the surface, around 53 times. This allows the lander to lose most of its kinetic energy in the upper atmosphere and then descend at a relatively slow rate through the rest of the atmosphere where it will be collecting scientific data.

During this phase the lander must possess adequate aerodynamic characteristics to achieve mission goals. The lander must possess both static and dynamic aerodynamic stability. There are mission requirements on the angular rotation rate of the lander so that cameras can obtain clear images, free of motion blur. Additionally the angle at which the lander impacts the surface cannot be too high or the energy absorption system will not function as designed. The lander makes use of devices to improve these aerodynamic characteristics and aid in impact worthiness. These devices can be seen in Figure 1.2. A drag plate is used to keep the descent rate under $10 \mathrm{~m} / \mathrm{s}$ near the surface. A crushable energy absorber is used to cushion the impact with the planetary surface. Outriggers are to keep the lander upright upon impact. The lander is uncontrolled so it is up to the lander's passive stability characteristics to ensure the mission goals are accomplished.

\subsubsection{Lander}

Figure 1.2 shows the components that comprise the lander. The main component is a pressure vessel which houses all electronics and acts as a mounting point for all other components. A crushable energy absorber aids in the reduction of the shock of impact with the planetary surface upon landing. Five outriggers, mounted at equidistant angles around the pressure 
vessel, act to stabilize the lander upon impact with the surface and provide additional drag in the terminal descent phase. A drag plate is mounted to the top of the pressure vessel and is used to control the descent rate and increase stability. There is an excavation arm that is mounted on the side of the lander that is intended to analyze soil samples. The lander, as designed, has a mass of around $850 \mathrm{~kg}$ and a maximum diameter of $3.9 \mathrm{~m}$.

\subsubsection{Wind Tunnel Testing}

Wind tunnel tests were conducted in support of the SAGE mission development with a purpose of exploring the mission design space of the drag plate and its relation to the drag and stability of the lander. These tests were conducted by Juan R. Cruz of NASA Langley Research Center (LaRC). Static wind tunnel tests were conducted to explore the drag and static stability of various configurations of the drag plate. The best configurations were selected for dynamic wind tunnel testing in the VST. The dynamic wind tunnel testing was conducted to obtain information on the dynamic motions of the lander. The present research exploits the data collected for one of the drag plate configurations used in the dynamic testing. More detailed information on both the static and dynamic wind tunnel tests is presented in Chapter 2.

\subsection{Thesis Outline}

This thesis is organized as follows:

- In Chapter 2 the wind tunnel tests conducted in support of the SAGE lander are discussed. The static and dynamic tests are described and the results presented. The dynamic test, being the most relevant to the present research, is presented in greater detail.

- In Chapter 3 the preprocessing of the raw data is examined. The smoothing routine is described and the equations required for the calculation of the state variables presented.

- In Chapter 4 the aerodynamic modeling is discussed. The method for determining the most appropriate model is examined and the final chosen model is presented. The parameter identification method is defined and the necessary equations developed. The methods to determine the accuracy of the identified parameters are presented.

- In Chapter 5 the results of the PID analyses on the SAGE wind tunnel data are presented and discussed. The parameters and their values are examined and validated against reconstructions of dynamic test runs not used in the PID analyses, static wind tunnel tests, and flight simulations.

- In Chapter 6, conclusions of the analysis are discussed and recommendations of further work given. 


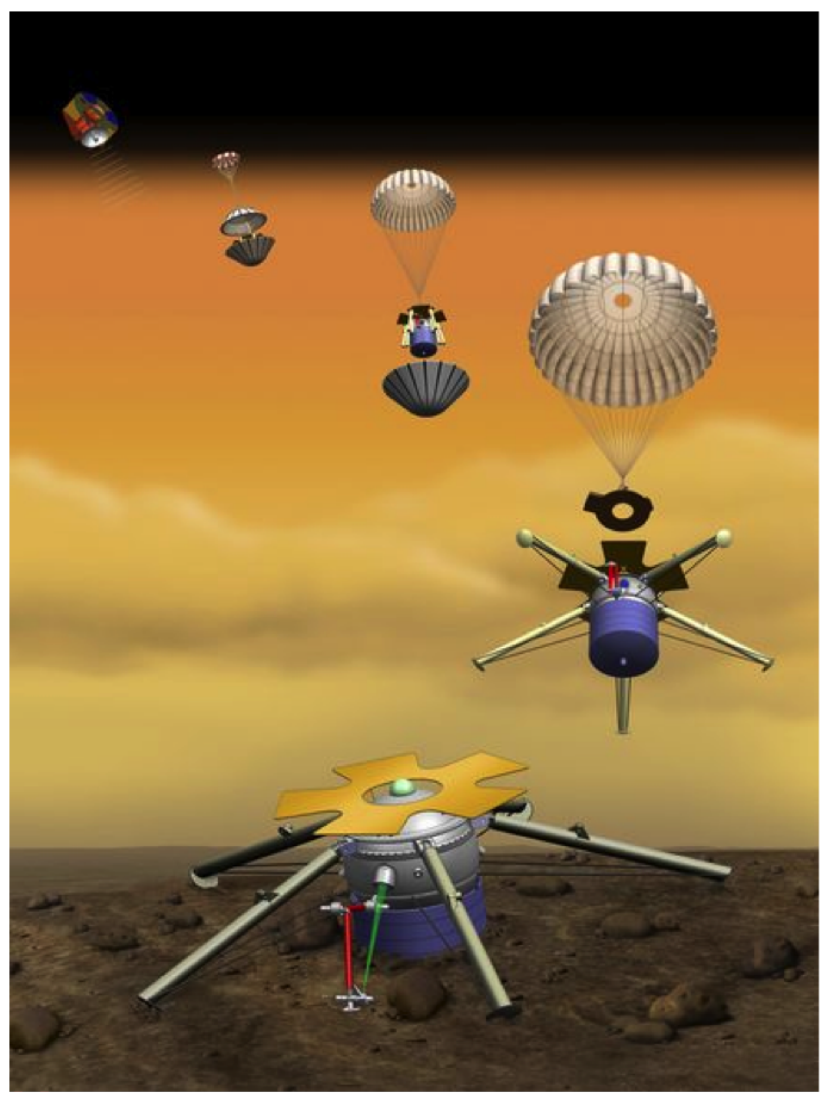

Figure 1.1: Artist's concept of the SAGE EDL sequence. [1]

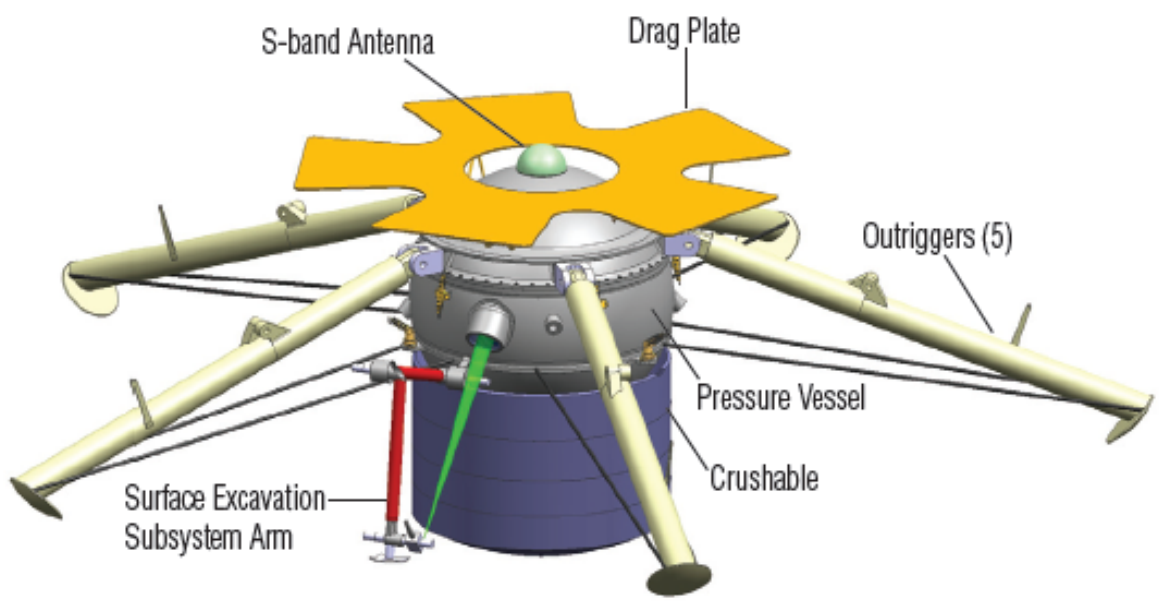

Figure 1.2: SAGE lander with major components labeled. Note that the excavation arm is in the operation position, it is stowed during flight. [1] 


\section{Chapter 2: Wind Tunnel Testing}

To evaluate the aerodynamic and dynamic characteristics of the SAGE lander it was subjected to both static and dynamic wind tunnel testing. . The purpose of these tests was to investigate the aerodynamic behavior of various configurations of the lander. The results were used to make lander configuration design decisions. The size and geometry of the drag plate was varied to determine which would yield the desired descent rate and acceptable dynamic characteristics. The presence and diameter of the outriggers were also varied in the static wind tunnel test. The static wind tunnel test was conducted prior to the dynamic test and served to downselect the configurations for dynamic testing. Of the 39 configurations tested in the static test only 5 were tested in the VST. For the purposes of this thesis, only one of the five will be analyzed; the model configuration with the large drag plate. Although these tests were not conducted by the author as a part of the research for this thesis, this chapter is included for completeness.

\subsection{Dynamic Wind Tunnel Testing}

\subsubsection{Test Facility}

\section{Specifications}

The NASA 20-ft VST is a closed-throat, annular-return vertical wind tunnel that operates with air at ambient conditions (sea level). Figure 2.1 shows a schematic of the wind tunnel. The test section has a 12-sided regular polygon cross section that is $20 \mathrm{ft}$ across and $25 \mathrm{ft}$ high. A three bladed fan at the top of the test section forces air through turning vanes to the annular-return passage. A flow straitening grid mitigates flow angularity, from the merging of the airstreams around the return passage, upon the airstream entering the test section. The airstream velocity in the test section can reach $85 \mathrm{ft} / \mathrm{s}$ and a dynamic pressure of 8.6 psf. For more details on the VST see Table 2.1. The test section is partially lined with observation windows which may be opened to launch models into the airstream. Safety nets on the top and bottom of the test section keep the model contained and the walls are padded to reduce model damage in case of accidental contact with the walls.

${ }^{*}$ Both the static and dynamic wind tunnel tests were conducted by Juan R. Cruz of NASA LaRC. The test plan and report for each test were used as references for this chapter. These documents are internal NASA documents and are unpublished. 
Table 2.1: NASA LaRC VST specifications. [2]

\begin{tabular}{ll}
\hline Test section dimensions & $25 \mathrm{ft}$ high by $20 \mathrm{ft}$ wide \\
Cross section & 12 -sided regular polygon, $300 \mathrm{ft}^{2}$ \\
Contraction ratio & $5: 1$ \\
Max speed & $85 \mathrm{ft} / \mathrm{s}$ \\
Max dynamic pressure & $8.6 \mathrm{psf}$ \\
Max Reynolds number & $0.55 \times 10^{6}$ per ft \\
Drive power & $400 \mathrm{hp}$ continuous and $1300 \mathrm{hp}$ in short bursts \\
\hline
\end{tabular}

\section{Tether}

The dynamically scaled models used in the VST are built to meet mass and geometric requirements, and can be quite delicate. Contact with the test section walls could damage the model causing a delay in testing and requiring model repairs. As a safety precaution a lightweight, small diameter tether is sometimes attached to the models so they may be pulled away from danger. From the model, the tether is threaded through a ring suspended in the center of the test section with guy wires. The tether is connected to an electric winch which is activated if the model approaches the wall. The tether geometry and components are shown in Figure 2.1. The aerodynamic drag on the tether keeps it out of the way of the free-flying model. For SAGE lander testing, this tether system was used. The effect of the tether on the free flight characteristics of the SAGE lander model is discussed in section 2.1.2.

\section{Operation}

During testing the model is flown at the level of the observation windows. The tunnel velocity is controlled by an operator and is varied in an attempt to keep the model vertically centered in the test section. To begin a wind tunnel run, the model is suspended in the center of the test section by the tether and the tunnel is turned on. The drag on the model lifts it in front of the observation windows and data acquisition is initiated. After the run the model is returned to a suspended state and data acquisition ended.

\subsubsection{Lander Scale Model}

\section{Scaling}

A dynamically scaled model of the lander was constructed for testing in the VST. The model was geometrically a quarter scale model of the SAGE lander and its mass properties were Froude scaled to give similitude with the conditions that the SAGE lander would encounter at $20 \mathrm{~km}$ altitude in the Venus atmosphere. Table 2.2 presents the dimensions and mass properties of the full-scale and scale model SAGE landers. 
Table 2.2: SAGE full-scale and model landers.

\begin{tabular}{llll}
\hline & Full-Scale & Model-Scale & Units \\
\hline Scale & 100 & 25 & $\%$ \\
Pressure vessel diameter, $d$ & $0.850(2.7887)$ & $0.2125(0.6972)$ & $\mathrm{m}(\mathrm{ft})$ \\
Pressure vessel frontal area, $S$ & $0.567(6.108)$ & $0.0355(0.3818)$ & $\mathrm{m}^{2}\left(\mathrm{ft}^{2}\right)$ \\
Outrigger diameter, $d_{o r}$ & $101.6(4.000)$ & $25.4(1.00)$ & $\mathrm{mm}(\mathrm{in})$ \\
Lander mass, $m$ & $840.4(57.59)$ & $0.7873(0.0539)$ & $\mathrm{kg}(\mathrm{slug})$ \\
Roll mass moment of inertia, $I_{x x}$ & $3.609 \times 10^{8}$ & $2.113 \times 10^{4}$ & $\mathrm{~kg} \cdot \mathrm{mm}^{2}$ \\
& $2.662 \times 10^{2}$ & $1.558 \times 10^{-2}$ & $\mathrm{slug} \cdot \mathrm{ft}^{2}$ \\
Pitch mass moment of inertia, $I_{y y}$ & $2.357 \times 10^{8}$ & $1.380 \times 10^{4}$ & $\mathrm{~kg} \cdot \mathrm{mm}^{2}$ \\
& $1.738 \times 10^{2}$ & $1.018 \times 10^{-2}$ & $\mathrm{slug} \cdot \mathrm{ft}^{2}$ \\
Yaw mass moment of inertia, $I_{z z}$ & $2.356 \times 10^{8}$ & $1.379 \times 10^{4}$ & $\mathrm{~kg} \cdot \mathrm{mm}^{2}$ \\
& $1.738 \times 10^{2}$ & $1.017 \times 10^{-2}$ & $\mathrm{slug} \cdot \mathrm{ft}^{2}$ \\
\hline
\end{tabular}

\section{Construction}

The model was constructed from 3-D printed plastic and Mylar. The pressure vessel, excavator arm, crushable energy absorber and outrigger end pads were fabricated as individual components from 3-D printed plastic. The cylindrical outriggers were fabricated from rolled Mylar sheet; its ends were braced by thin nylon line. The drag plates are fabricated from 3-D printed frames covered with Mylar. The drag plates can be replaced, allowing for testing with different drag plates using the same model core (i.e., pressure vessel, energy absorber, and outriggers). The model mass properties were adjusted by a system including an internal movable mass, and small masses attached to the outrigger end pads.

\subsubsection{Test Description}

Five SAGE configurations were tested during the course of the dynamic wind tunnel testing. For this thesis the only configuration analyzed is for a large drag plate with outriggers. Figure 2.2 shows the SAGE lander model configuration selected for use in the PID analysis.

\section{Unperturbed and Perturbed Test Runs}

For each configuration tested there were runs where the model was perturbed, by an external force, and left unperturbed, allowing the model to determine its own motion. The SAGE lander displays significant damping, as a result on runs where the lander is unperturbed the total angle of attack seldom rises above six degrees. In an effort to obtain aerodynamic data at higher angles of attack the model was perturbed. To perturb the model, a wind tunnel technician contacts the side of the model with a pole to increase the angle of attack. This event causes the model to oscillate and often requires the operator to throttle the tunnel up or down to keep the model vertically centered in the test section. Several perturbations 
could be performed during a given run. Sometimes, after a perturbation, the model needed to be rescued by activating the tether to keep the model from striking the wall. In such a case the run was terminated.

\section{Tether Force Determination}

The tether imposes forces and moments on the model that are absent in true free flight. In past programs the effect of the tether has been neglected in post-test analyses. This assumption is only valid if the magnitude of the tether forces and moments imposed on the model are sufficiently small as compared to the aerodynamic and inertial forces and moments encountered by the model. In determining the contributions of small dynamic moments to the stability of the vehicle the tether may have a comparatively large effect. The tether acts on the model as if it were a drogue parachute, affecting the stability of the model. To differentiate the tether force from the model aerodynamic forces and moments more information needs to be known about the magnitude of the tether force.

To determine the tether force a streamlined lead mass was attached to the tether in place of the model. The wind tunnel was powered up until the mass was lifted. The velocity was adjusted to hold the mass in equilibrium at a desired vertical position. At this point the velocity was recorded. This process was repeated with five different masses (see Figure 2.3). The length of the tether was the same from run to run. The aerodynamic drag on the mass was assumed to be negligible. The equilibrium conditions, at which the velocity is recorded, means that the tether force was assumed to be equal to the weight of the streamlined mass. The results of this test, given in Table 2.3 in Section 2.1.5, were used to generate a tether force model which is detailed in Appendix B.2.

\subsubsection{Data Acquired}

\section{Coordinate Systems}

The tunnel fixed coordinate system is oriented such that the positive $X$ axis is directed vertically downwards. The $Y$ and $Z$ axes form the horizontal plane. Figure 2.1 shows the tunnel fixed coordinate system in a cross section of the tunnel. Note that the coordinate system is adapted from a standard coordinate system which has the $Z$ axis pointing downwards. The data collected using the standard coordinate system suffered from singularity issues with the Euler angles necessitating the change to the tunnel fixed coordinate system.

There are two body fixed coordinate systems used in the PID analysis: a geometric model fixed coordinate system and a center of mass fixed coordinate system. The geometric model

fixed coordinate system origin is defined as the geometric center of the pressure vessel. The $x$ axis is out the nose of the vehicle, the $y$ axis is along the outrigger opposite the excavation arm. The geometric model fixed coordinate system is used for data collection by the motion capture system. The center of mass fixed coordinate system origin is at the center of mass and its axes are parallel to those of the geometric model fixed coordinate system. The center of mass is defined with respect to the geometric model fixed coordinate system. The two 
systems were necessitated by the fact that model was constructed with the ability to change the center of mass along the axis of symmetry. The position data, collected at the geometric origin, is converted to the center of mass in preprocessing (see Chapter 3) for analysis. The Euler angles and rates are the same regardless of where the coordinate system is centered. Figure 2.4 shows the geometric model fixed coordinate system.

\section{Motion Capture System}

The position and attitude of the vehicle was recorded via a motion capture system at a rate of $150 \mathrm{~Hz}$. The model was fitted with reflective dots which were observed by a number of cameras in the tunnel. The tunnel's computer calculates the position of each reflective dot by triangulating the view of the cameras. The coordinates of the geometric model fixed coordinate system origin relative to the tunnel fixed coordinate system is then calculated based on the known locations of the reflective dots on the model. In a similar manner the Euler angles are determined. The data collected by the motion capture system are:

- The position of the origin of the geometric model fixed coordinate system relative to the tunnel fixed coordinate system, $(X, Y$ and $Z)$.

- The Euler angles of the vehicle in an $x-y-z$ rotation sequence, $\left(R_{x}, R_{y}\right.$ and $\left.R_{z}\right)$.

\section{Other Recorded Variables}

In addition to the position and attitude data other variables important to the analysis are recorded at the same $150 \mathrm{~Hz}$ data rate. These variables are:

- The wind dynamic pressure in the tunnel, $q_{W}$.

- The free stream static pressure in the tunnel, $p_{s}$.

- The magnitude of the upward wind velocity, $V_{W}$.

- The static temperature in the tunnel, $T_{s}$.

\section{Documentation Camera}

The runs were documented with a high definition video camera. In circumstances where the motion of the vehicle cannot be fully understood from the motion data the video data can be observed to see the actual motion of the model. The video data can also be synchronized with the motion data to better determine the time window of the data to be used in the PID analysis. 


\subsubsection{Test Results}

\section{Lander}

Several minutes of data were collected using the large drag plate with outriggers configuration. The data were organized into two blocks: unperturbed and perturbed. Figures 2.5a$2.5 \mathrm{~d}$ show the typical results for an unperturbed run of 60 seconds. Note that the pitch and yaw $\left(R_{y}\right.$ and $R_{z}$ ) Euler angles are very small and rarely exceed seven degrees (or an angle of attack of approximately six degrees upon further analysis). Data at higher Euler angles were collected during the perturbed runs. Figures 2.6a-2.6d show a typical perturbation. The data used for the PID analysis is collected immediately after the contact with the lander model has ended. At this point the lander model is at its maximum state of perturbation. The dynamic wind tunnel data used in the PID analysis is displayed in Appendix A.

The recovery period immediately following the perturbation gives insight to the lander's dynamic damping capabilities. Figure $2.6 \mathrm{~b}$ shows that the oscillations due to the perturbation, in which the lander model reached $\sim 30$ degrees, are damped out after $\sim 2$ oscillations. The lander model then enters a period of very low angle oscillations, returning to the behavior observed in an unperturbed state.

\section{Tether Force}

The weight of each streamlined mass was recorded as well as the velocity of the wind tunnel. The dynamic pressure was calculated using an atmospheric density of $0.00229 \mathrm{slug} / \mathrm{ft}^{3}$. The weight of the masses was measured on a digital scale with an uncertainty of $\pm 0.0001 \mathrm{lb}$. The uncertainty in the velocity measurements was estimated to be $\pm 1 \mathrm{ft} / \mathrm{s}$. The magnitude of the tether force, $F_{t r}$, is assumed to equal to the weight of the streamlined mass. Table 2.3 shows the results of this test. These results were used to generate a tether force model which is detailed in Appendix B.2.

Table 2.3: Tether force wind tunnel experiment results. The weight numbers correspond to the weights shown in Figure 2.3 .

\begin{tabular}{lrrr}
\hline Weight \# & $F_{\text {tr }}(\mathrm{lb})$ & $V_{\infty}(\mathrm{ft} / \mathrm{s})$ & $q_{\infty}(\mathrm{psf})$ \\
\hline 1 & 0.0040 & 22 & 0.6 \\
2 & 0.0068 & 26 & 0.8 \\
3 & 0.0115 & 31 & 1.1 \\
4 & 0.0148 & 33 & 1.2 \\
5 & 0.0229 & 41 & 1.9 \\
\hline
\end{tabular}




\subsection{Static Wind Tunnel Testing}

\subsubsection{Test Facility}

The static wind tunnel test was conducted in the NASA Ames Research Center (ARC) Fluid Mechanics Laboratory's (FML) subsonic wind tunnel. The facility is an open-circuit indraft wind tunnel with a test section that measures 48 in tall by 32 in wide with a contraction ratio of 9:1. The tunnel is capable of an airstream velocity of $165 \mathrm{ft} / \mathrm{s}$ (Mach 0.15) with a Reynolds number of $1.1 \times 10^{6}$ per ft.

\subsubsection{Lander Scale Model}

An $11.58 \%$ scale model of the lander was used in the testing. Various sizes and configurations of the drag plate and outriggers were tested. Only the configuration that has similitude with the lander model configuration used in the PID analysis is reported here.

\subsubsection{Test Description}

The model lander was mounted to a L-sting fixed to a floor-mounted turntable. This table was rotated to change the azimuth of the model with respect to the incoming freestream (see Figure 2.7). The roll angle of the model lander was adjusted to obtain data at various total angles of attack, $\alpha_{T}$, to show variation of the aerodynamic forces and moments on the model with respect to the total angle of attack clock angle, $\phi_{\alpha_{T}}$.

\subsubsection{Data Acquired}

All three force and moment components were obtained from the internal wind tunnel balance for multiple values of $\alpha_{T}$ and $\phi_{\alpha_{T}}$. The force and moment components reduce to the aerodynamic coefficients: $C_{X}, C_{Y}, C_{Z}, C_{l_{o}}, C_{m_{o}}, C_{n_{o}}$. By imposing the assumption of axisymmetry, an axisymmetric model was devised in which the value varies with $\alpha_{T}$ but remained constant with $\phi_{\alpha_{T}}$.

\subsubsection{Test Results}

The axisymmetric aerodynamic model data, relevant to the model under consideration in this thesis, are shown in Figure 2.8. These data are used to partially validate results of the parameter identification analyses. 


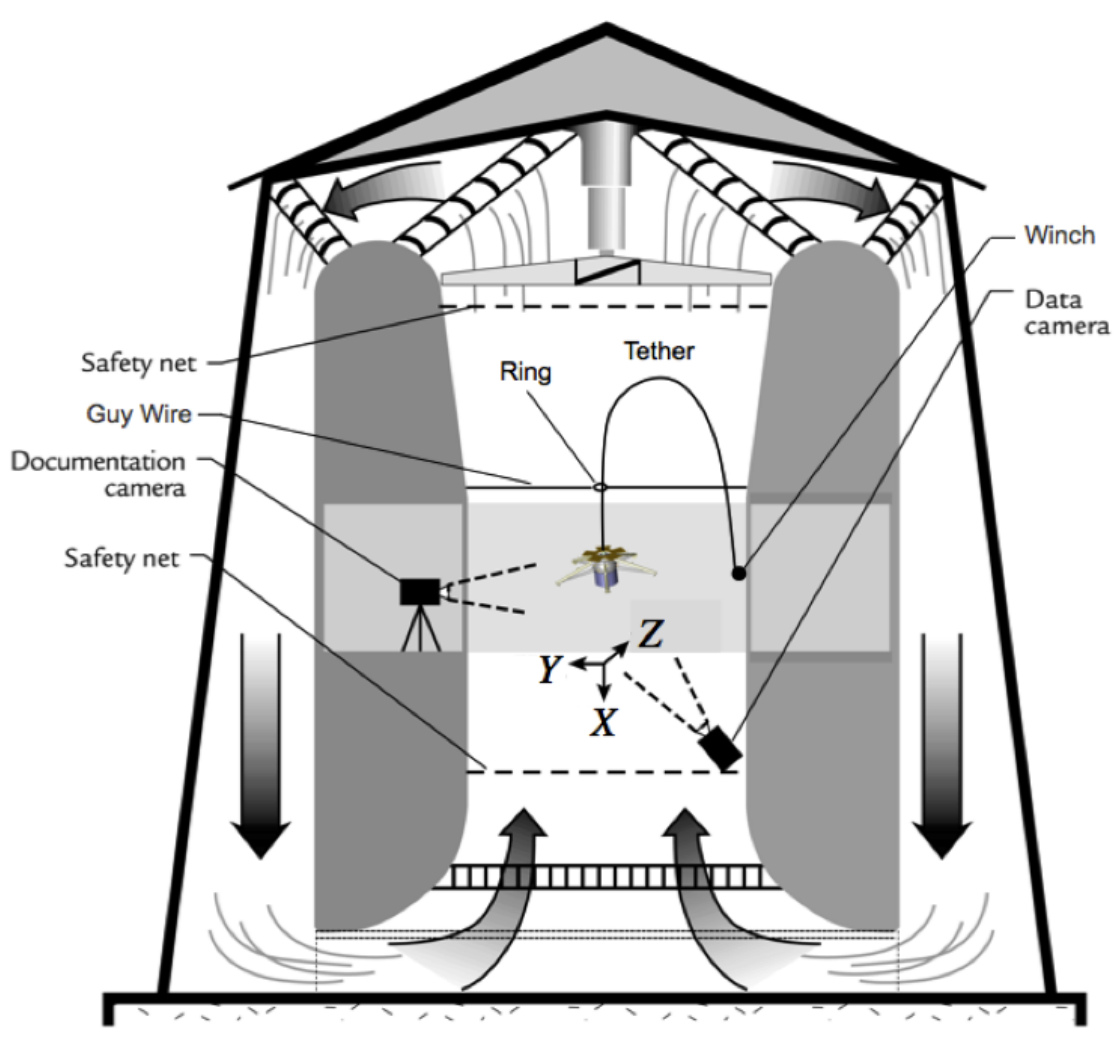

Figure 2.1: NASA LaRC 20-ft VST. Adapted from image provided by NASA LaRC.

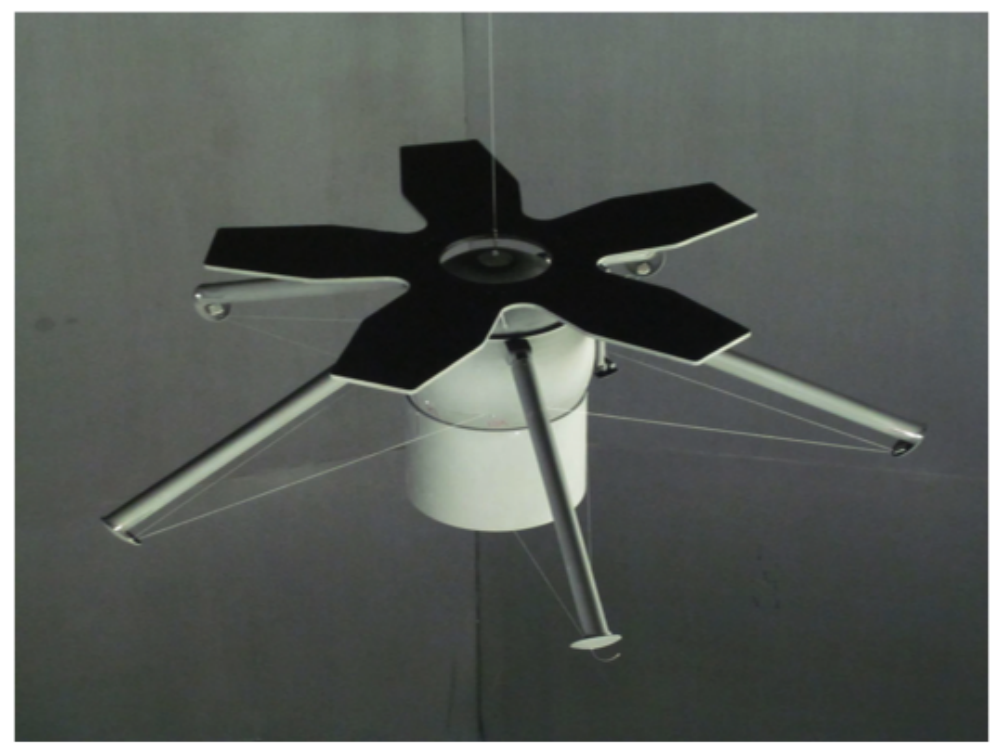

Figure 2.2: SAGE lander dynamic wind tunnel Froude scaled model. Image provided by NASA LaRC. 

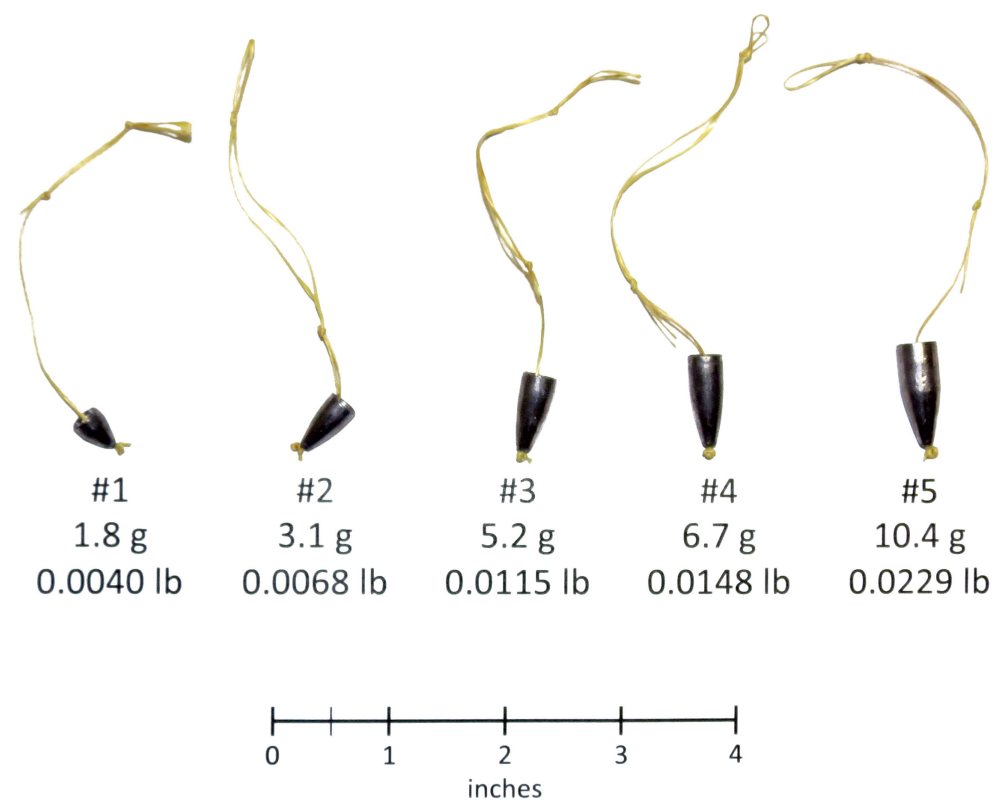

Figure 2.3: Tether force testing weights. Image provided by NASA LaRC.
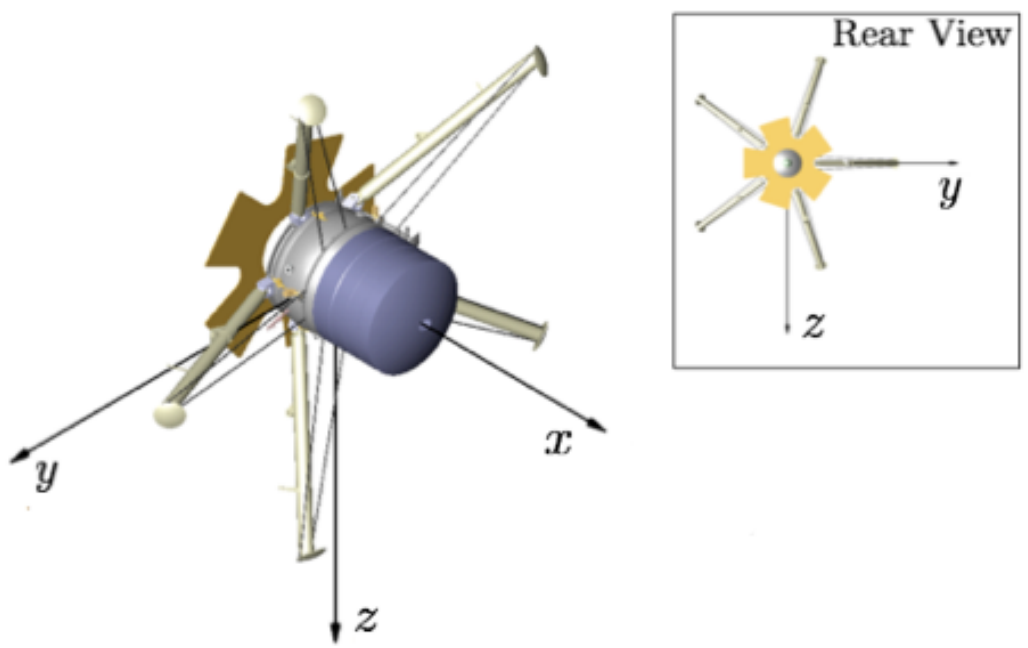

Figure 2.4: The geometric model-fixed coordinate system with origin at the geometric center of the pressure vessel. Adapted from image provided by NASA LaRC. 


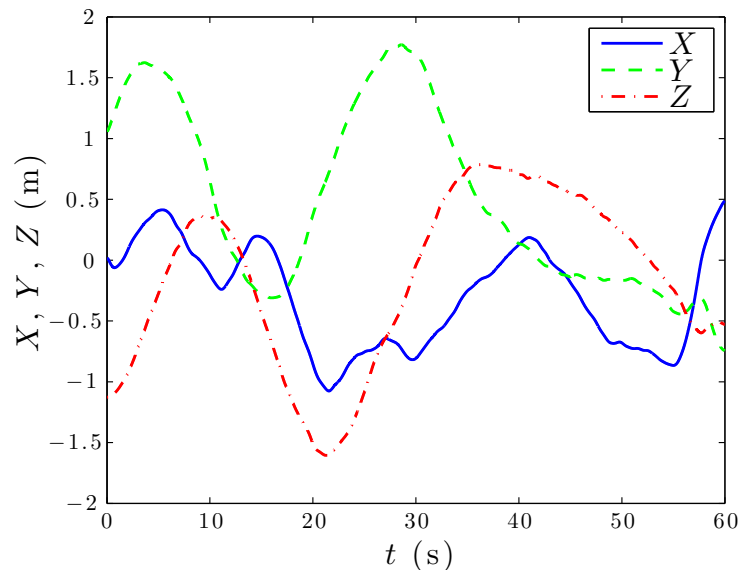

(a) $X, Y$ and $Z$ positions.

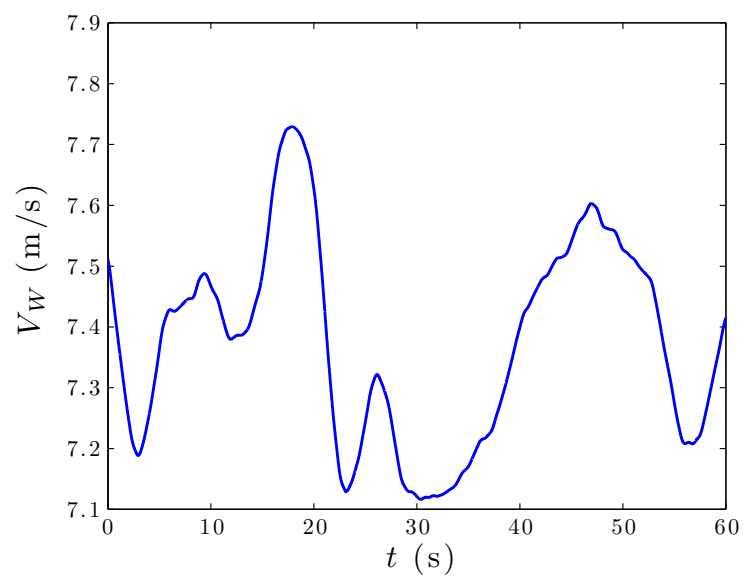

(c) Wind velocity magnitude.

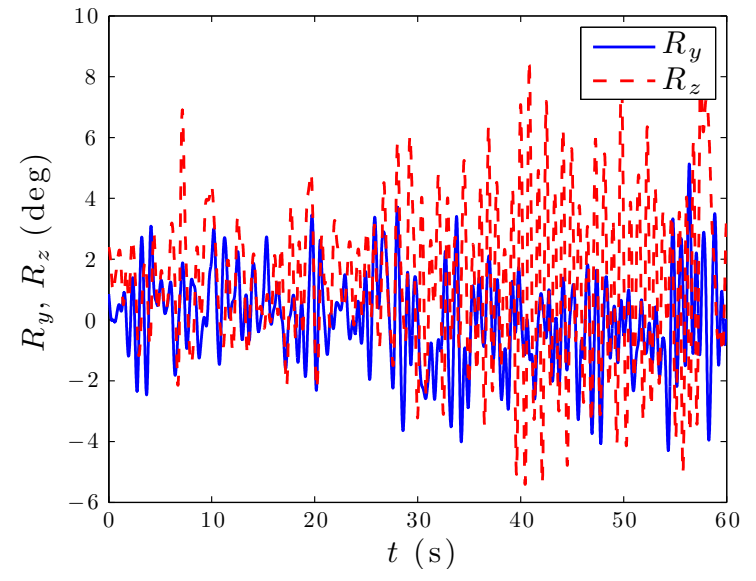

(b) $R_{y}$ and $R_{z}$ Euler angles.

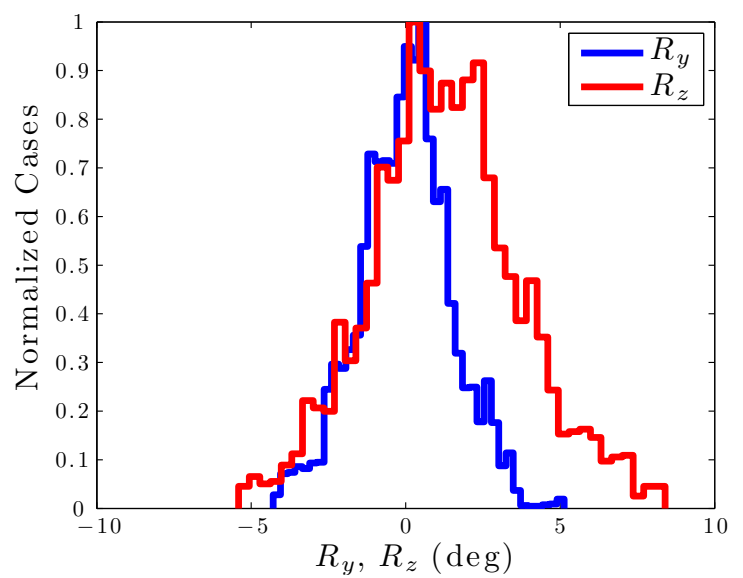

(d) Histogram of the $R_{y}$ and $R_{z}$ Euler angles.

Figure 2.5: Typical set of data collected during an unperturbed run. These data are from Test Block 2 Run 2. 


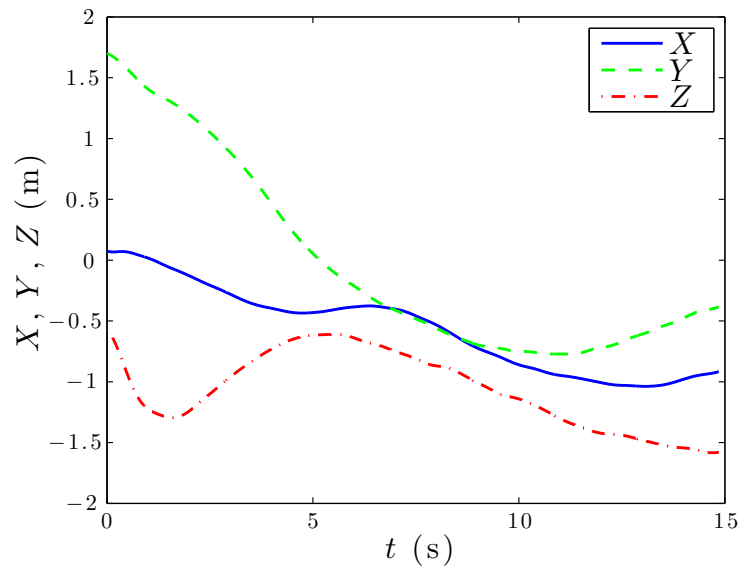

(a) $X, Y$ and $Z$ positions.

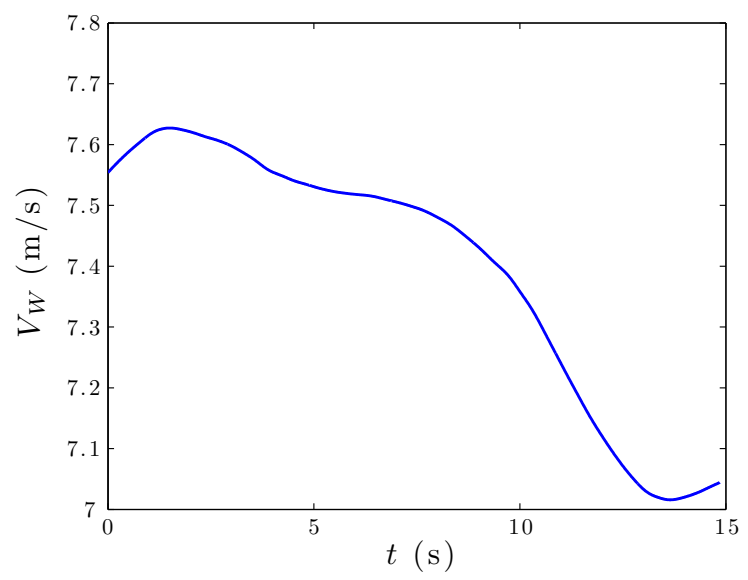

(c) Wind velocity magnitude.

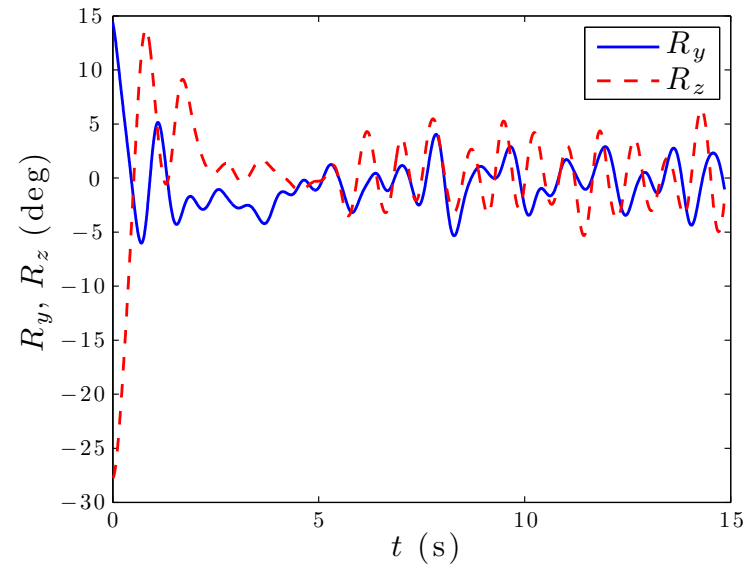

(b) $R_{y}$ and $R_{z}$ Euler angles.

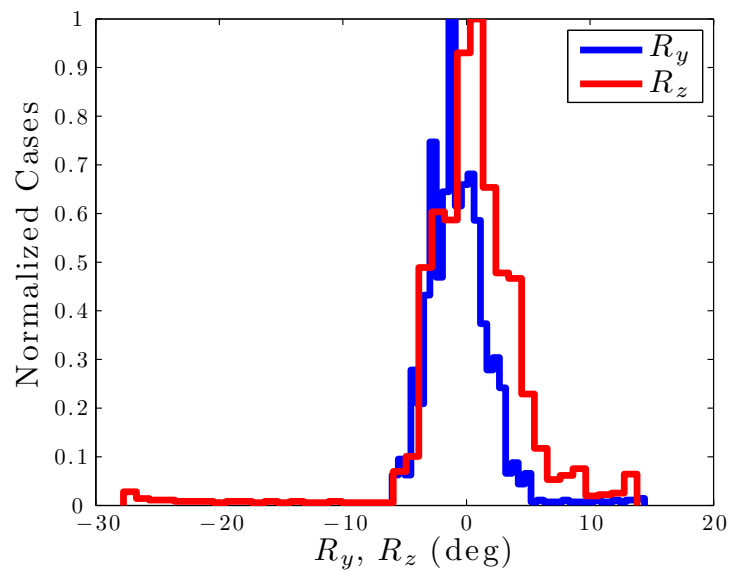

(d) Histogram of the $R_{y}$ and $R_{z}$ Euler angles.

Figure 2.6: Typical set of data collected during a perturbed run. These data are from Test Block 3 Run 2. 


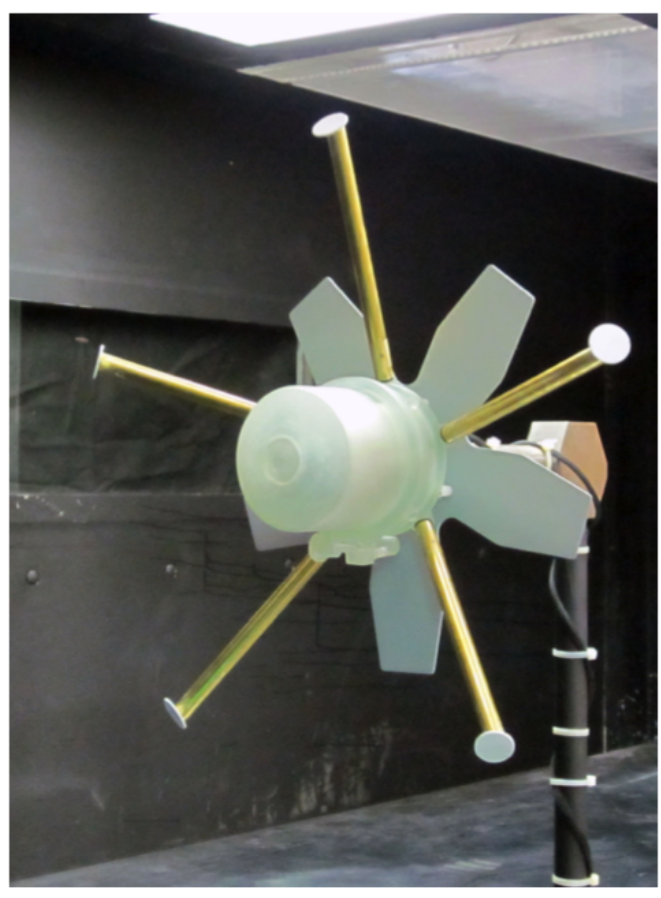

Figure 2.7: SAGE lander static wind tunnel scale model.

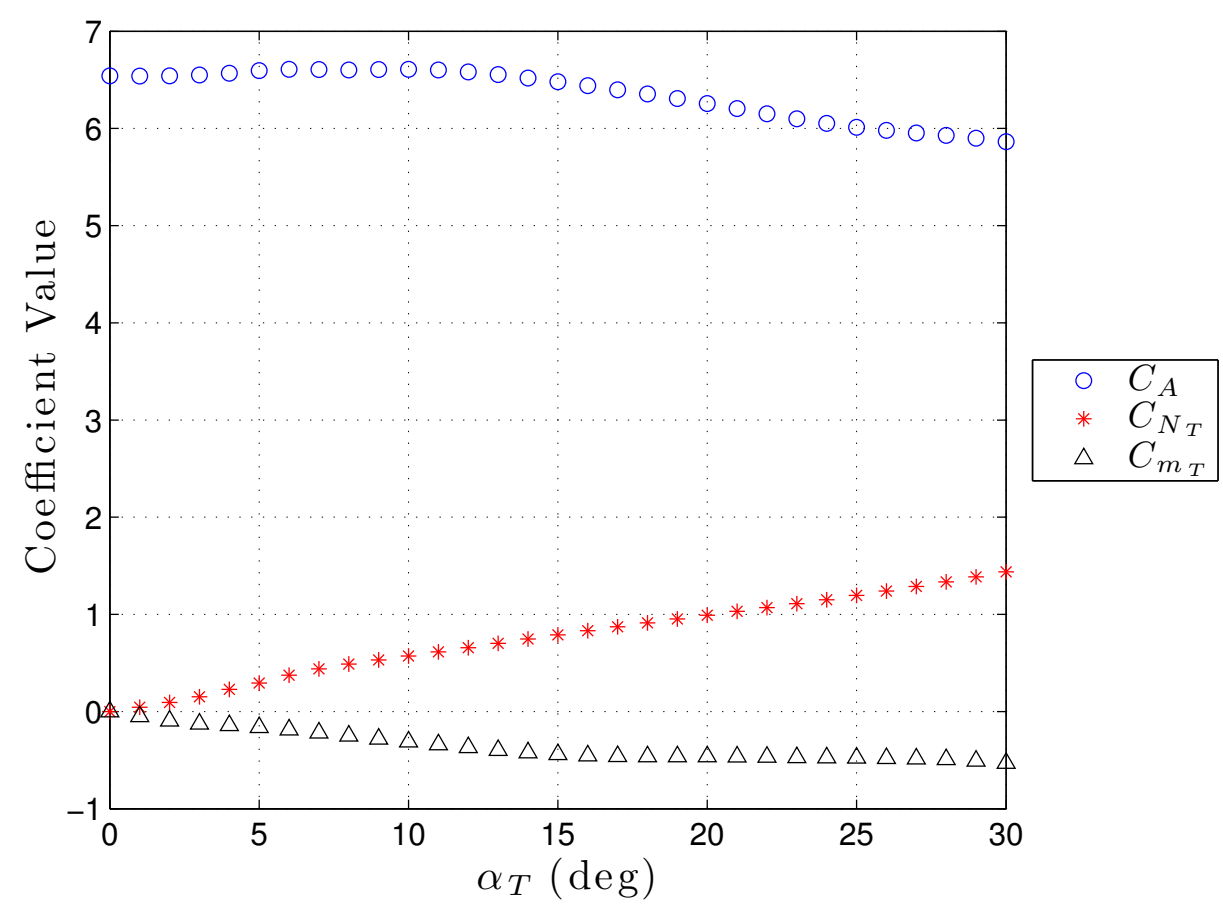

Figure 2.8: Axisymmetric static aerodynamic model derived from static wind tunnel data for the lander with the large drag plate. 


\section{Chapter 3: Data Preprocessing}

In order to estimate the aerodynamic coefficients required for the parameter identification analysis the state variables must be calculated from the data collected in the VST. To do this the velocities, accelerations, etc. of the lander must be determined in the inertial coordinate system and then converted to the body coordinate system. This means that the first and second time derivatives are required from the raw data signals. The raw wind tunnel data contained noise that yields unreliable derivatives when finite differencing is used. Therefore, the raw data signals must be smoothed to remove noise prior to the calculation of the time derivatives. This chapter will discuss the smoothing routine, calculation of the derivatives, and determination of the state variables.

\subsection{Global Smoothing Routine}

There are multiple methods available for separating noise from a signal. Global Fourier smoothing was chosen for this application for two reasons. First, the global view of the data is available to give the best estimate of the frequencies contained therein. Some filtering methods only use the past history to smooth the signal and there is an associated phase shift based on the cutoff frequency selected. Second, the smoothed signal is defined by an analytical equation for which closed form derivatives can be obtained thus giving accurate estimates of the time derivatives.

To show the process, consider the signal for the $X$ component of the position of the lander model for Test Block 2 Run 2 shown in Figure $3.1 \mathrm{a}$. The raw signal, $\check{X}_{i}$, contains $N$ data points at constant time interval, $\Delta t$, and is composed of the true signal, $X_{i}$, and noise, $\nu_{i}$.

$$
\check{X}_{i}=X_{i}+\nu_{i} \quad i=0,1,2, \ldots N-1
$$

The goal of the smoothing routine is to estimate $X_{i}$ from $\check{X}_{i}$. To do this the raw signal is rebuilt as a Fourier series. By examining the frequency spectrum of the Fourier series an estimate can be made of which frequencies are noise and which represent the true signal. A filter is designed to remove unwanted frequencies and a smooth signal is constructed. This smoothing routine is covered in detail in reference [3]. The SIDPAC software, included with reference [4], was implemented for the smoothing. This decision was made because the SIDPAC software had been verified and validated. 


\subsubsection{Fourier Reconstruction}

The raw signal, including noise, is reconstructed with a Fourier series. This series is a sum of sine and cosine terms at discrete frequencies and magnitudes that can replicate periodic functions. To obtain a periodic function from the raw signal the following transformation, shown in Eqs. (3.2) and (3.3), is applied.

$$
\begin{gathered}
\check{\xi}_{i}=\check{X}_{i}-\check{X}_{0}-i\left(\frac{\check{X}_{N-1}-\check{X}_{0}}{N-1}\right) \quad i=0,1,2, \ldots N-1 \\
\check{\xi}_{-i}=-\check{\xi}_{i} \quad i=0,1,2, \ldots N-1
\end{gathered}
$$

The transformation in Eq. (3.2) removes any linear trend present in the signal by setting the endpoints equal to zero. Eq. (3.3) reflects the signal about both its vertical and horizontal axes. These transformations are shown in Figures $3.1 \mathrm{~b}$ and $3.1 \mathrm{c}$. The result is a repeating signal the has the property that:

$$
\check{\xi}_{-(N-1)}=\check{\xi}_{0}=\check{\xi}_{N-1}=0
$$

Note that $\check{\xi}_{i}$ is an odd periodic function, thus it can be represented as a Fourier series consisting of only sine terms. This sine series has the form given in Eq. (3.5).

$$
\tilde{\xi}_{i}=\sum_{k=1}^{N-1} b_{k} \sin \left[\omega_{k} i \Delta t\right] \quad i=0,1,2, \ldots N-1
$$

where the amplitudes of the terms, $b_{k}$, are,

$$
b_{k}=\frac{2}{N-1} \sum_{i=1}^{N-2} \check{\xi}_{i} \sin \left[k \pi\left(\frac{i}{N-1}\right)\right] \quad k=0,1,2, \ldots N-1
$$

and the angular frequencies, $\omega_{k}$, are,

$$
\omega_{k}=\frac{k \pi}{(N-1) \Delta t} \quad k=0,1,2, \ldots N-1
$$

The discrete frequencies, $f_{k}$, and periods, $T_{k}$, can be calculated using Eqs. (3.8) and (3.9) respectively. The maximum frequency as calculated in Eq. (3.8) is the Nyquist frequency.

$$
\begin{array}{rlrl}
f_{k} & =\frac{\omega_{k}}{2 \pi}=\frac{k}{2(N-1) \Delta t} & k=0,1,2, \ldots N-1 \\
T_{k}=\frac{1}{f_{k}}=\frac{2(N-1) \Delta t}{k} & k=0,1,2, \ldots N-1
\end{array}
$$

The raw signal can be reconstructed via Eq. (3.10) by adding the linear trend back to the periodic function.

$$
\tilde{X}_{i}=\tilde{\xi}_{i}+\check{X}_{0}+i\left(\frac{\check{X}_{N-1}-\check{X}_{0}}{N-1}\right) \quad i=0,1,2, \ldots N-1
$$


The reconstructed signal, $\tilde{X}_{i}$, is shown in Figure $3.1 \mathrm{~d}$. The signal still contains the noise that was present in the raw data. To remove the noise a filter must be designed and implemented in the Fourier series. This process is described in the next section.

\subsubsection{Filter Design and Implementation}

A filter is used to remove the noise from the signal and obtain an estimate of the true signal, $X_{i}$. A Wiener filter is used in the current analysis because it allows for some cushion in the cutoff frequency selection. The series can be truncated at the cutoff frequency but often the line separating the true signal from the noise is not clearly identifiable. The Wiener filter tapers the signal over this region and takes some of both sides of the cutoff frequency into consideration.

The first step in the filter design is selecting a cutoff frequency, $f_{o}$. By examining a plot of $\left|b_{k}\right|$ versus $f_{k}$ a cutoff frequency can be determined. Figure 3.2a shows the frequency spectrum for $\check{X}_{i}$. Figure $3.2 \mathrm{~b}$ is a zoomed in view of Figure $3.2 \mathrm{a}$, the cutoff frequency of 1.5 $\mathrm{Hz}$ was selected for this signal. The filter can then be designed specifically for this signal. The Wiener filter has the mathematical form given in Eq. (3.11). Figure 3.2c shows the shape of the filter as a function of the frequency. Note that at the cutoff frequency the filter's value is 0.5 .

$$
\Phi_{k}=\frac{\eta_{k}^{2}}{\eta_{k}^{2}+1} \quad k=0,1,2, \ldots N-1
$$

where,

$$
\eta_{k}=\left\{\begin{array}{ll}
\left(f_{o} / f_{k}\right)^{3} & \text { if }\left(f_{o} / f_{k}\right)^{3}>0.01 \\
0 & \text { if }\left(f_{o} / f_{k}\right)^{3} \leq 0.01
\end{array} \quad k=0,1,2, \ldots N-1\right.
$$

Note that the exponent in Eq. (3.12) determines the sharpness of the transition is across the cutoff frequency. The exponent can be increased for a shaper transition. The filter is truncated after 0.01 or $1 \%$ of the signal amplitude. The smoothed signal, $X_{i}$, is constructed using Eq. (3.13) by inserting the filter term into the Fourier series expansion.

$$
X_{i}=\xi_{i}+\check{X}_{0}+i\left(\frac{\check{X}_{N-1}-\check{X}_{0}}{N-1}\right) \quad i=0,1,2, \ldots N-1
$$

where the smoothed periodic function is,

$$
\xi_{i}=\sum_{k=1}^{N-1} \Phi_{k} b_{k} \sin \left[\omega_{k} i \Delta t\right] \quad i=0,1,2, \ldots N-1
$$

The signal noise, $\nu_{i}$, is determined from Eq. (3.15) and gives insight to the noise level of the smoothed signal. Figure 3.3 compares the raw and smoothed signals and shows the noise. Note that the Figures $3.3 \mathrm{a}$ and $3.3 \mathrm{~b}$ look very similar but the smoothed signal yields cleaner and more consistent derivatives. See the Section 3.2 for the calculation of the smoothed derivatives. It is assumed that the noise has a Gaussian distribution with an expected value 
and variance as in Eqs. (3.16) and (3.17). The expected value of the noise should be very close to zero. For the current analysis it was assumed that an expected value of the noise on the order of $10^{-4}$ or smaller was adequate. If this condition is not met the smoothing routine is repeated with a higher cutoff frequency.

$$
\begin{gathered}
\nu_{i}=\check{X}_{i}-X_{i} \quad i=0,1,2, \ldots N-1 \\
\bar{\nu}=\frac{1}{N} \sum_{i=0}^{N-1} \nu_{i} \\
s_{\nu}^{2}=\frac{1}{N-1} \sum_{i=0}^{N-1}\left(\nu_{i}-\bar{\nu}\right)
\end{gathered}
$$

The process described by Eqs. (3.2)-(3.17) should be repeated for all variables collected in the VST as described in section 2.1.5.

\subsubsection{Cutoff Frequencies}

The cutoff frequencies for the motion data is given in Table 3.1. These values were determined to meet the requirements of expected value of the noise. The quality of the derivatives were taken into account in the selection of the cutoff frequency as well. The signals for static pressure and temperature are typically given a constant value as variability in the signal is very small over the course of the wind tunnel run.

Table 3.1: Cutoff frequencies for the motion data collected in the VST.

\begin{tabular}{cc}
\hline Variable & $f_{o}(\mathrm{~Hz})$ \\
\hline$X$ & 1.5 \\
$Y$ & 1.5 \\
$Z$ & 1.5 \\
$R_{x}$ & 1.5 \\
$R_{y}$ & 2.5 \\
$R_{z}$ & 2.5 \\
$V_{W}$ & 1.5 \\
\hline
\end{tabular}

\subsection{Smoothed Derivatives}

The first and second time derivatives of the position and attitude variables as well as the first derivative of the wind velocity are required for the equations of motion. Because the smoothed signal is a Fourier series the closed form solution of the time derivative can be 
determined by taking the derivative of Eq. (3.13). These derivatives are shown in Eqs. (3.18) and (3.19).

$$
\begin{gathered}
\dot{X}_{i}=\sum_{k=0}^{N-1} \omega_{k} \Phi_{k} b_{k} \cos \left(\omega_{k} i \Delta t\right)+\left(\frac{\check{X}_{N-1}-\check{X}_{0}}{N-1}\right) \quad i=0,1,2, \ldots N-1 \\
\ddot{X}_{i}=-\sum_{k=0}^{N-1} \omega_{k}^{2} \Phi_{k} b_{k} \sin \left(\omega_{k} i \Delta t\right) \quad i=0,1,2, \ldots N-1
\end{gathered}
$$

\subsection{Calculation of State Variables}

The following equations are used to calculate the state variables for the model lander from the smoothed wind tunnel data and its first and second derivatives. The angular velocity $(p, q, r)$ and the angular acceleration $(\dot{p}, \dot{q}, \dot{r})$ in the body fixed coordinate system are determined from the recorded Euler angles' first $\left(\dot{R}_{x}, \dot{R}_{y}, \dot{R}_{z}\right)$ and second $\left(\ddot{R}_{x}, \ddot{R}_{y}, \ddot{R}_{z}\right)$ time derivatives using Eqs. (3.20)-3.23). The rotational translation matrix $\left[T_{1}\right]$ is used for the transformation. Note that Eq. $(3.22)$ is the derivative of Eq. (3.20).

$$
\begin{aligned}
& \left\{\begin{array}{c}
p \\
q \\
r
\end{array}\right\}=\left[T_{1}\right]\left\{\begin{array}{c}
\dot{R}_{x} \\
\dot{R}_{y} \\
\dot{R}_{z}
\end{array}\right\} \\
& {\left[T_{1}\right]=\left[\begin{array}{ccc}
\cos R_{y} \cos R_{z} & \sin R_{z} & 0 \\
-\cos R_{y} \sin R_{z} & \cos R_{z} & 0 \\
\sin R_{y} & 0 & 1
\end{array}\right]} \\
& \left\{\begin{array}{c}
\dot{p} \\
\dot{q} \\
\dot{r}
\end{array}\right\}=\left[\dot{T}_{1}\right]\left\{\begin{array}{c}
\dot{R}_{x} \\
\dot{R}_{y} \\
\dot{R}_{z}
\end{array}\right\}+\left[T_{1}\right]\left\{\begin{array}{c}
\ddot{R}_{x} \\
\ddot{R}_{y} \\
\ddot{R}_{z}
\end{array}\right\} \\
& {\left[\dot{T}_{1}\right]=\left[\begin{array}{ccc}
-\dot{R}_{y} \sin R_{y} \cos R_{z}-\dot{R}_{z} \cos R_{y} \sin R_{z} & \dot{R}_{z} \cos R_{z} & 0 \\
\dot{R}_{y} \sin R_{y} \sin R_{z}-\dot{R}_{z} \cos R_{y} \cos R_{z} & -\dot{R}_{z} \sin R_{z} & 0 \\
\dot{R}_{y} \cos R_{y} & 0 & 0
\end{array}\right]}
\end{aligned}
$$

The velocity at the center of mass is defined in vector notation by Eq. (3.24). The location of the center of mass is specified by the vector $\mathbf{r}_{c m}$ from the center of the pressure vessel (i.e., the origin of the geometric body fixed coordinate system) to the center of mass. The components of $\mathbf{r}_{c m}$ are $x_{c m}, y_{c m}$ and $z_{c m}$.

$$
\mathbf{U}=\mathbf{U}_{o}+\omega \times \mathbf{r}_{c m}
$$

The center of mass velocity components $(u, v, w)$ are calculated from Eq. (3.25) based on the velocity of the origin of the geometric model fixed coordinate system $\left(u_{o}, v_{o}, w_{o}\right)$, defined by Eq. 3.26. 


$$
\left\{\begin{array}{c}
u \\
v \\
w
\end{array}\right\}=\left\{\begin{array}{l}
u_{o} \\
v_{o} \\
w_{o}
\end{array}\right\}+\left[\begin{array}{ccc}
0 & -r & q \\
r & 0 & -p \\
-q & p & 0
\end{array}\right]\left\{\begin{array}{l}
x_{c m} \\
y_{c m} \\
z_{c m}
\end{array}\right\}
$$

where,

$$
\left\{\begin{array}{c}
u_{o} \\
v_{o} \\
w_{o}
\end{array}\right\}=\left[T^{B E F}\right]^{T}\left\{\begin{array}{c}
\dot{X} \\
\dot{Y} \\
\dot{Z}
\end{array}\right\}
$$

The acceleration $(\dot{u}, \dot{v}, \dot{w})$ at the center of mass is determined by Eq. (3.27). The transformation matrix, $\left[T^{B E F}\right]$, and its derivative, $\left[\dot{T}^{B E F}\right]$, are defined in Appendix B in Eqs. (B.13) and (B.42).

$$
\left\{\begin{array}{c}
\dot{u} \\
\dot{v} \\
\dot{w}
\end{array}\right\}=\left\{\begin{array}{c}
\dot{u}_{o} \\
\dot{v}_{o} \\
\dot{w}_{o}
\end{array}\right\}+\left[\begin{array}{ccc}
0 & -\dot{r} & \dot{q} \\
\dot{r} & 0 & -\dot{p} \\
-\dot{q} & \dot{p} & 0
\end{array}\right]\left\{\begin{array}{l}
x_{c m} \\
y_{c m} \\
z_{c m}
\end{array}\right\}
$$

where,

$$
\left\{\begin{array}{c}
\dot{u}_{o} \\
\dot{v}_{o} \\
\dot{w}_{o}
\end{array}\right\}=\left[\dot{T}^{B E F}\right]^{T}\left\{\begin{array}{c}
\dot{X} \\
\dot{Y} \\
\dot{Z}
\end{array}\right\}+\left[T^{B E F}\right]^{T}\left\{\begin{array}{c}
\ddot{X} \\
\ddot{Y} \\
\ddot{Z}
\end{array}\right\}
$$




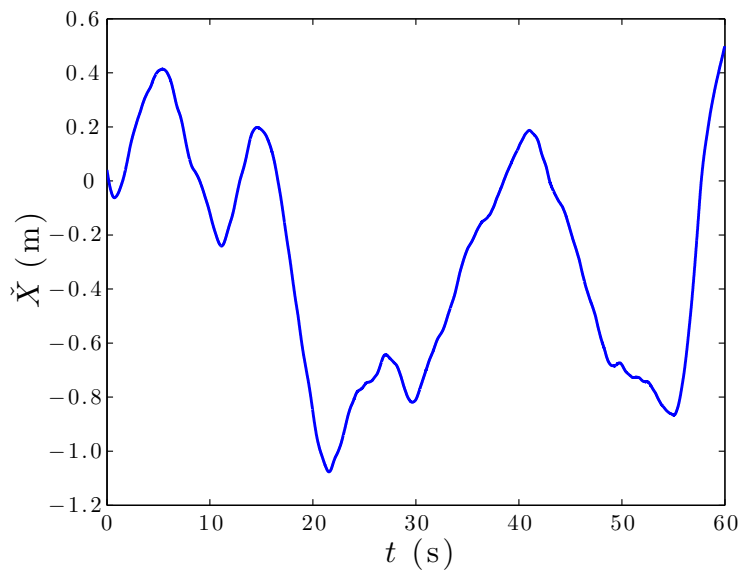

(a) Raw signal, $\check{X}$.

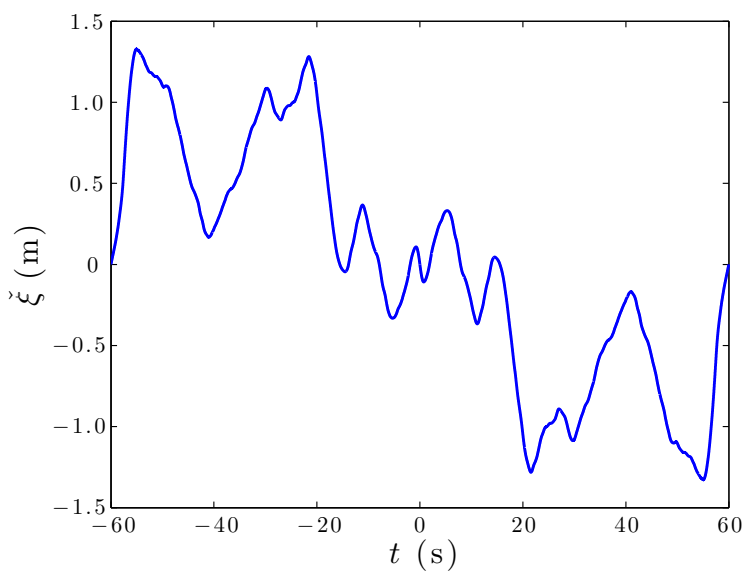

(c) Signal after Eq 3.3 .

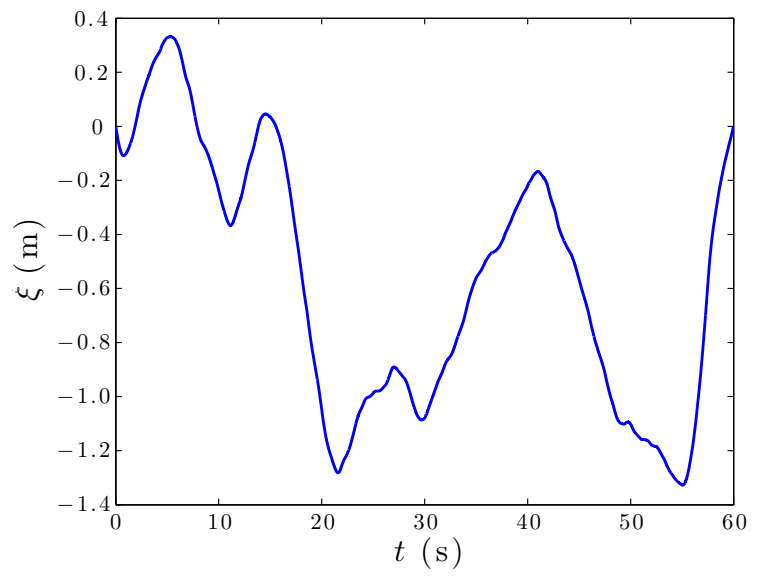

(b) Signal after Eq. (3.2).

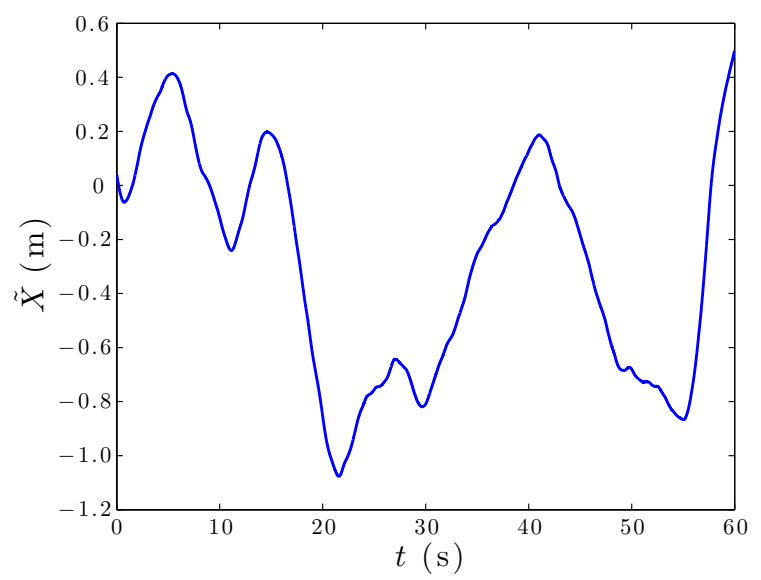

(d) Reconstructed signal, $\tilde{X}$.

Figure 3.1: Reconstruction of a signal via Fourier series. 


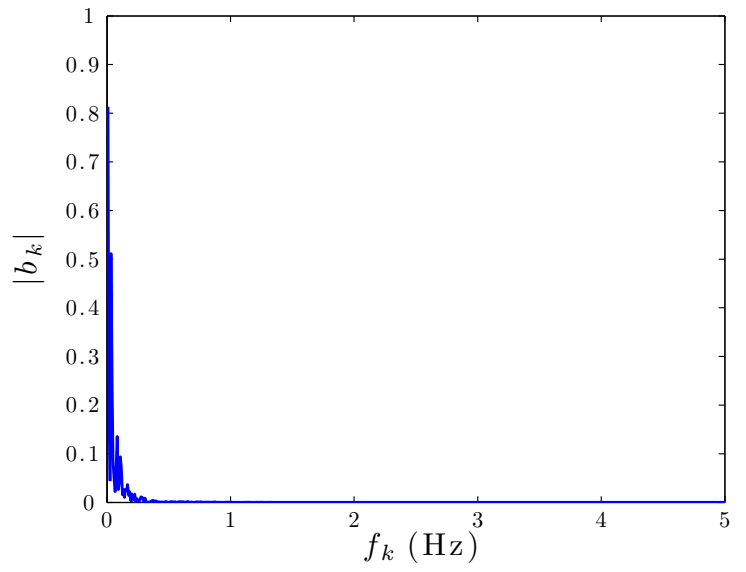

(a) Frequency spectrum for $\tilde{X}$ position data.

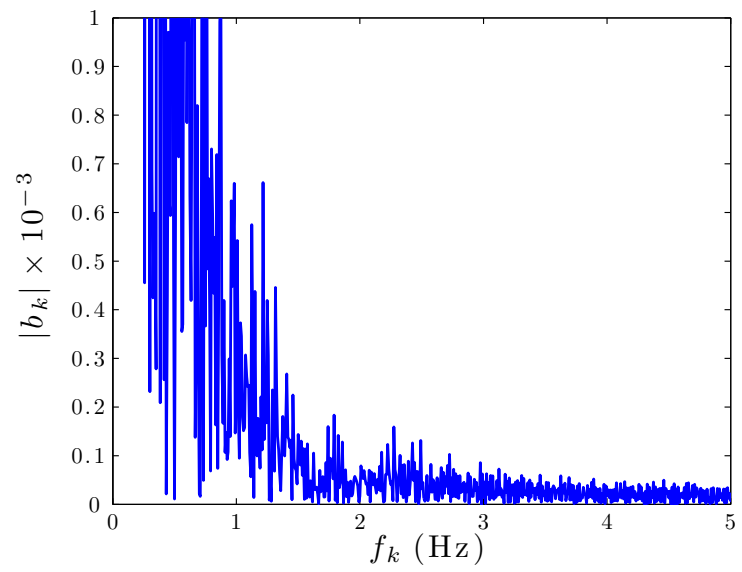

(b) Zoomed in frequency spectrum.

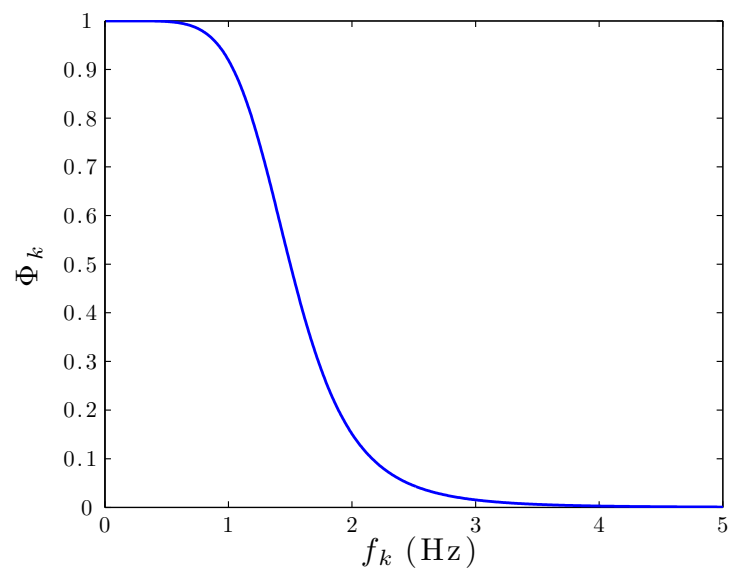

(c) Wiener filter shape.

Figure 3.2: Frequency spectrum and filter shape. The selected cutoff frequency for this signal is $1.5 \mathrm{~Hz}$. 


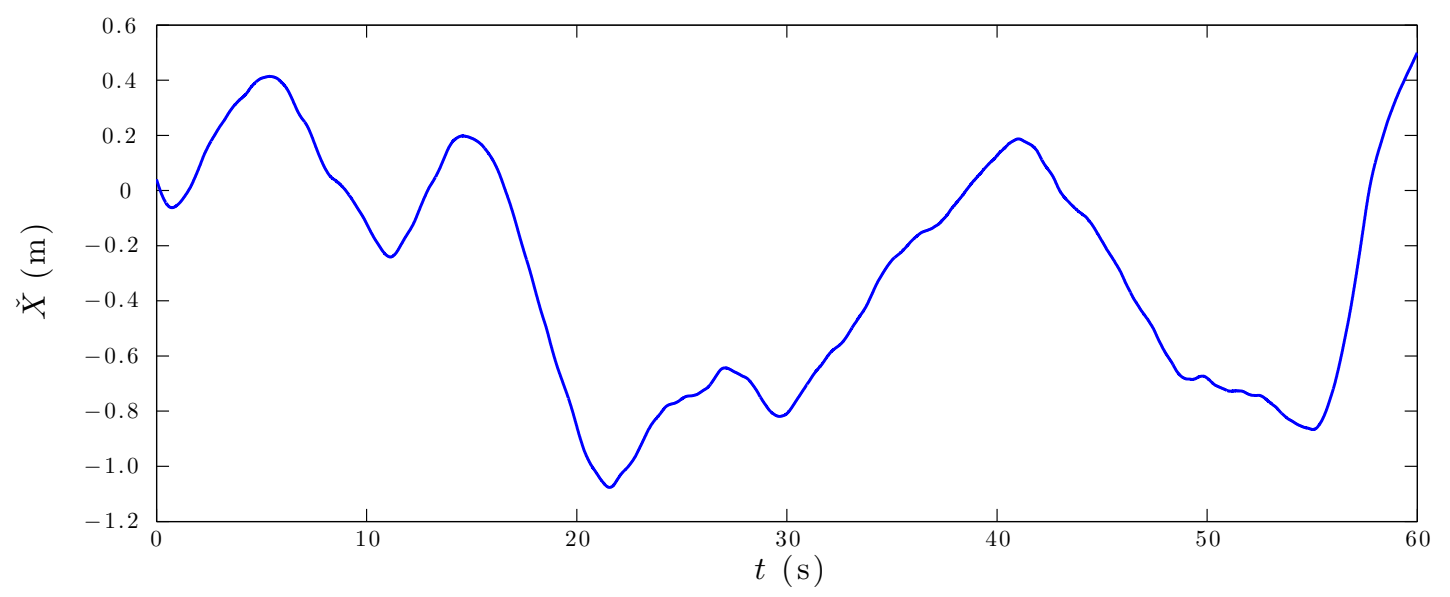

(a) Raw wind tunnel data, $\check{X}$.

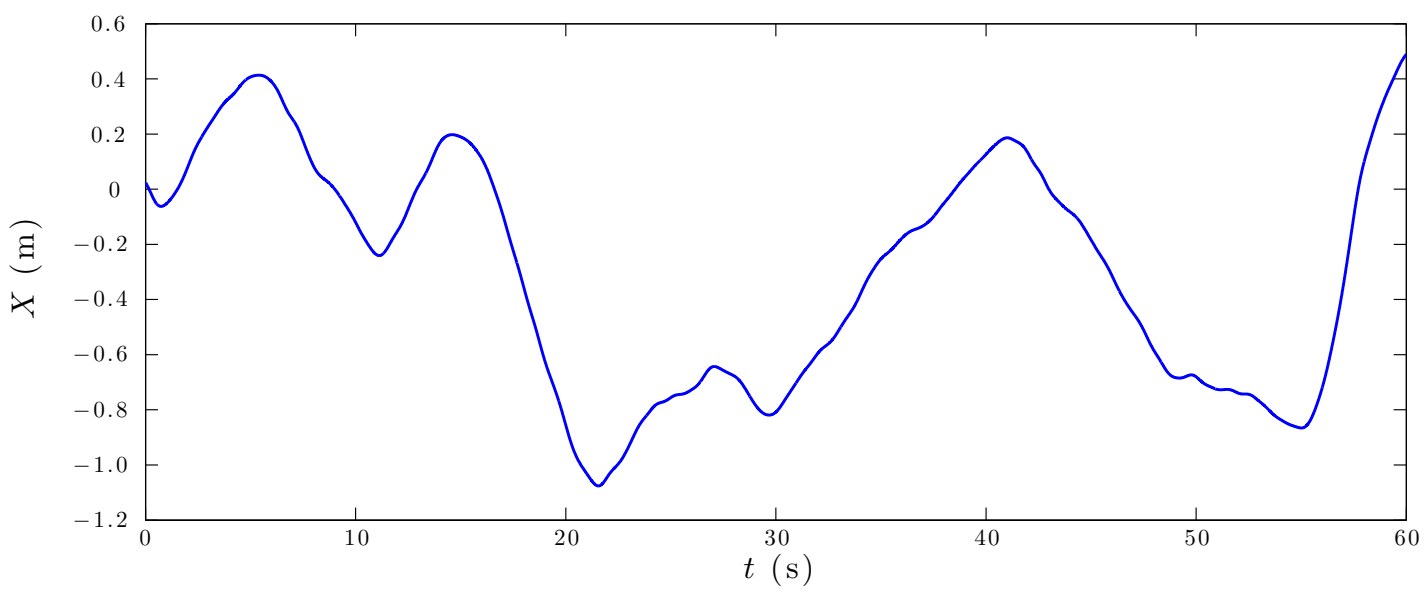

(b) Smoothed wind tunnel data, $X$.

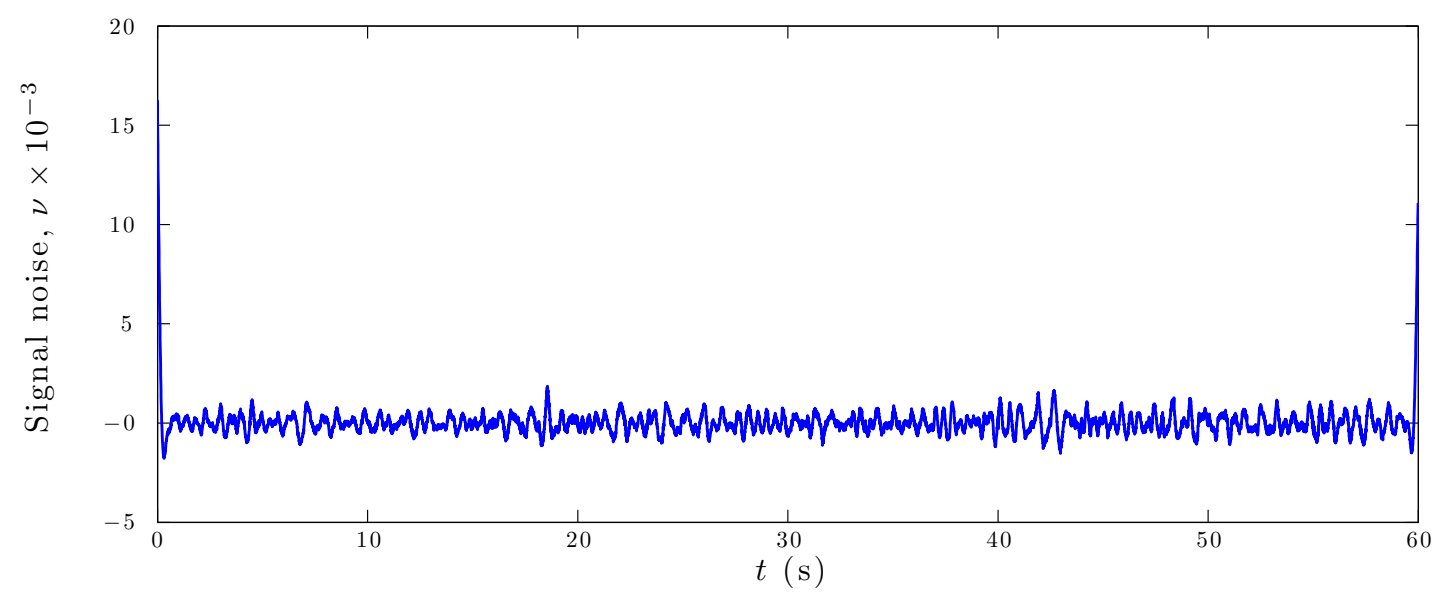

(c) Noise filtered out of $\check{X}$ signal.

Figure 3.3: Comparison of raw and smoothed data. 


\title{
Chapter 4: System Identification
}

A system identification analysis involves both aerodynamic model structure determination and estimation of the parameters therein. For the SAGE lander, the mathematical form of the aerodynamic model was chosen based on a priori knowledge but model optimization was performed which yielded insight to the aerodynamic behavior. The PID analysis was conducted to estimate the values of constant parameters contained within the aerodynamic model for the lander. This is done by optimizing the parameter values such that the difference between the experimental and modeled aerodynamic coefficients is minimized. In this chapter the aerodynamic model and the parameter estimation method used is discussed.

\subsection{Aerodynamic Modeling}

\subsubsection{Model Structure Determination}

Much work has been devoted to understanding the aerodynamic behavior of blunt entry vehicles in the subsonic flight regime. Most of the research is centered around the dynamic stability of the vehicle in the pitch and yaw planes. These planes are important because they are the most likely to induce tumbling of the vehicle and thus, are of primary interest in the PID analysis. Mitcheltree, et al. in Reference [5] point out the following about the pitch and yaw aerodynamics:

\begin{abstract}
"The oscillatory motion of a blunt entry vehicle in subsonic terminal descent is analogous to that of a nonlinear mass-spring-damper system. In this analogy, static stability corresponds to the spring stiffness and dynamic stability corresponds to the damper's characteristics. The dynamic damping of an aeroshell is highly nonlinear and can be destabilizing at small angle-of-attack and stable for larger angles which (if statically stable) leads to limit cycle behavior."
\end{abstract}

The SAGE lander displays this type of limit cycle behavior. The lander is known to be statically stable throughout the total angle of attack range up to $30^{\circ}$ from the static wind tunnel testing. Therefore, any instabilities come from the dynamic stability of the lander. The lander possesses high levels of static and dynamic stability at higher angles of attack achieved when perturbed but is noticeably less stable at lower angles of attack. The static stability of the lander as observed from the static wind tunnel test data is nearly linear. 
It is assumed that the static stability is a odd function of the angle of attack or sideslip and is therefore represented as polynomial expansion of odd terms. Likewise, the dynamic stability is assumed to be an even function of angle of attack or sideslip and is represented as a polynomial expansion of even terms. Due to the near axisymmetry of the lander, the values of the aerodynamic parameters obtained in the pitch and yaw expansions should have similar values. The two are solved separately and checked against each other for consistency.

Since the results of the static wind tunnel test were known beforehand, the form of the static force coefficients were tailored to the available data. The axial force coefficient varies with the total angle of attack. The total normal force similarly varies with the total angle of attack and can be split into the $y$ and $z$ components using the total angle of attack clock angle. Due to the lander's near axisymmetry, the values of aerodynamic parameters for the normal and side forces should be similar. The roll moment of the vehicle is assumed to have a constant static component and a damping component.

A model optimization analysis was conducted in an attempt to mathematically determine an optimal formulation of the aerodynamic model based on the VST data. A multivariate orthogonal function generation code, contained in the SIDPAC software included with reference [4], was used to determine which regressor variables had the highest correlation with the aerodynamic coefficient. The user could then include or exclude any regressor in the current model and the coefficient would be reconstructed. Fit error of the model would decrease with the addition of each regressor to a point. Theoretically, there exists an optimal number of parameters in a model that minimizes the mean squared error of the model without obtaining an over-parameterized model. A problem that was encountered was that there was no consistency between runs for the regressors with the highest correlation to the coefficient. A regressor that seemed very important for one run seemed to have little to no impact on the next run. Many of the models generated with this code would make no physical sense and when used in a simulation - the lander would immediately tumble. This analysis was therefore abandoned. Conclusions were drawn from this analysis and are discussed in Chapter 6.

\subsubsection{Aerodynamic Model}

The aerodynamic model used for the SAGE lander model for the PID analysis is representative of the research discussed above. All aerodynamics are modeled at the origin of the geometric model fixed coordinate system and its corresponding axes. The static aerodynamic models use the total angle of attack, $\alpha_{T}$, and total angle of attack clock angle, $\phi_{\alpha_{T}}$, to define wind angle as an alternate to angle of attack, $\alpha$, and angle of sideslip, $\beta$. These variables are defined in Appendix B. The model in each of the six axes of motion are assumed to be as follows:

\section{Axial Force}

$C_{A}$ is strongly correlated with the total angle of attack and is assumed not to possess a dynamic component. It is assumed to be modeled as seen in Eq. (4.1). The only aerodynamic 
parameter to be identified is $C_{A_{T, 0}}$ and represents the axial force at $\alpha_{T}=0$. This simple model agrees well with the static wind tunnel data.

$$
C_{A}=C_{A_{T, 0}} \cos \alpha_{T}
$$

\section{Normal and Side Forces}

The $C_{Y}$ and $C_{N}$ coefficients are related due to the nearly axisymmetric nature of the SAGE lander. Like the axial force, the normal and side forces are assumed to have no dynamic component. The total normal force coefficient is calculated using Eq. (4.2) and is the opposite sign of the total side force due to the sign conventions used for entry vehicles. The $y$ and $z$ components are determined by using the total angle of attack clock angle as seen in Eq. (4.3) and (4.4). The aerodynamic parameters $C_{N_{\sin \alpha_{T}}}$ and $C_{N_{\sin ^{3} \alpha_{T}}}$ are determined by the PID code.

$$
\begin{gathered}
C_{N_{T}}=C_{N_{\sin \alpha_{T}}} \sin \alpha_{T}+C_{N_{\sin ^{3} \alpha_{T}}} \sin ^{3} \alpha_{T} \\
C_{Y}=-C_{N_{T}} \sin \phi_{\alpha_{T}} \\
C_{N}=C_{N_{T}} \cos \phi_{\alpha_{T}}
\end{gathered}
$$

Note that this model has the property that,

$$
C_{N_{T}}=\sqrt{C_{N}^{2}+C_{Y}^{2}}
$$

An experimental value for $C_{N_{T}}$ can be obtained by the property shown in Eq. (4.5). The PID analysis is then performed on Eq. (4.2) and one set of parameters is obtained for both $C_{N}$ and $C_{Y}$. For low angle of attack cases, such as unperturbed cases, the cubic term may be dropped from the model because nonlinearities at low angles are sufficiently small.

\section{Roll Moment}

The aerodynamic roll moment coefficient is assumed to have a both static and dynamic components. The static component, $C_{l_{o, T}}$, is constant and the dynamic component, $C_{l_{p}}$, is a function of the non-dimensional roll rate. This model will allow the model to spin in one direction until the dynamic component counteracts it, reaching a steady state roll rate. This behavior is seen in the wind tunnel data. The aerodynamic parameters $C_{l_{o, T}}$ and $C_{l_{p}}$ are determined by the PID code.

$$
C_{l_{o}}=C_{l_{o, T}}+C_{l_{p}}\left[p\left(\frac{d}{2 V_{\infty}}\right)\right]
$$




\section{Pitch and Yaw Moments}

Like the normal and side forces the pitch and yaw moments are related by calculating a total static moment, $C_{m_{o, T}}$, and then splitting it into components with the total angle of attack clock angle. The static moment coefficient curve is an odd function that is represented as a cubic in Eq. 4.7). Due to sign conventions the total static moments about the $y$ and $z$ axes have opposite signs. The damping terms, $C_{m_{q+\dot{\alpha}}}$ and $C_{n_{r-\dot{\beta}}}$, are assumed to be functions of the angles of attack and sideslip respectively, see Eqs. (4.8) and (4.9). The $C_{m_{o}}$ and $C_{n_{o}}$ moment coefficients are the sum of the static and dynamic components given in Eqs. (4.10) and (4.11). The aerodynamic parameters $C_{m_{\sin \alpha_{T}}}, C_{m_{\sin ^{3} \alpha_{T}}}, C_{m_{1}}, C_{m_{2}}, C_{n_{1}}$, and $C_{n_{2}}$ are determined by the PID code.

$$
\begin{gathered}
C_{m_{o, T}}=C_{m_{\sin \alpha_{T}}} \sin \alpha_{T}+C_{m_{\sin ^{3} \alpha_{T}}} \sin ^{3} \alpha_{T} \\
C_{m_{q+\dot{\alpha}}}=C_{m_{1}}+C_{m_{2}} \alpha^{2} \\
C_{n_{r-\dot{\beta}}}=C_{n_{1}}+C_{n_{2}} \beta^{2} \\
C_{m_{o}}=C_{m_{o, T}} \cos \phi_{\alpha_{T}}+C_{m_{q+\dot{\alpha}}}\left[\left(\frac{q+\dot{\alpha}}{2}\right)\left(\frac{d}{2 V_{\infty}}\right)\right] \\
C_{n_{o}}=-C_{m_{o, T}} \sin \phi_{\alpha_{T}}+C_{n_{r-\dot{\beta}}}\left[\left(\frac{r-\dot{\beta}}{2}\right)\left(\frac{d}{2 V_{\infty}}\right)\right]
\end{gathered}
$$

Unlike the normal and side forces, the pitch and yaw moment coefficients, 4.10 and (4.11) respectively, must be solved individually. Due to the axisymmetric assumptions already stated, $C_{m_{1}} \simeq C_{n_{1}}$ and $C_{m_{1}} \simeq C_{n_{1}}$ is assumed to be true. The two sets of parameters identified for $C_{m}$ and $C_{n}$ respectively. The set deemed superior is selected as the value of the parameters for both. The cubed term may be disregarded for wind tunnel runs with very low angles of attack where the coefficient curve is nearly linear.

\subsection{Parameter Identification}

There are many parameter estimation methods available of varying complexity. The best method will depend on the mathematical form of the aerodynamic model and the available statistical information about the aerodynamic parameters. If the model is nonlinear with respect to the parameters then the best method would be a multidimensional solver such as the simplex method. If the uncertainty of the aerodynamic coefficients or the parameters is known then a maximum likelihood method would be best. For the current case an ordinary least squares estimator is used because the aerodynamic model is linear with respect to the aerodynamic parameters and the uncertainty of the coefficients and the parameters is unknown.

Regardless of the parameter identification method used, an objective function is to be minimized. This objective function, $J$, is the error between the experimentally determined 
aerodynamic coefficient, $\mathbf{C}_{\text {exp }}$, and the modeled aerodynamic coefficient, $\mathbf{C}$, which is a function of the aerodynamic parameters, p. This is seen in Eq. 4.12).

$$
J(\mathbf{p})=\frac{1}{2}\left(\mathbf{C}_{e x p}-\mathbf{C}(\mathbf{p})\right)^{T}\left(\mathbf{C}_{\exp }-\mathbf{C}(\mathbf{p})\right)
$$

The time history of $\mathbf{C}_{\exp }$ is determined from the VST data using the flight mechanics equations in Appendix B. The parameter vector, $\mathbf{p}$, is the optimization variable. This vector contains $n_{p}$ parameters to be identified. The parameter identification method presented in this chapter are covered in further detail in Chapter 5 of Reference [4] and in Reference 6]. The SIDPAC software, included with reference [4, was implemented for the parameter identification. This decision, like the smoothing, was made because the SIDPAC software had been verified and validated.

\subsubsection{Ordinary Least Squares Estimator}

The aerodynamic model used for the lander is linear with respect to the parameters therefore the model can be represented and solved via matrix algebra. The modeled aerodynamic coefficients can then be defined as follows:

$$
\mathbf{C}(\mathbf{p})=\mathbf{R} \mathbf{p}
$$

Where, $\mathbf{R}$ is the regressor matrix, of size $n_{p} \times N$, made up of experimentally determined variables, and $\mathbf{p}$ is the parameter vector, of size $1 \times n_{p}$, containing the aerodynamic parameters that are to be estimated. By inserting Eq. 4.13 into the objective function shown in Eq. (4.12) the new ordinary least squares objective function is obtained.

$$
J(\mathbf{p})=\frac{1}{2}\left(\mathbf{C}_{e x p}-\mathbf{R} \mathbf{p}\right)^{T}\left(\mathbf{C}_{\exp }-\mathbf{R} \mathbf{p}\right)
$$

The minimum of the cost function is where $\mathrm{d} J(\mathbf{p}) / \mathrm{d} \mathbf{p}=0$. Solving then for $\mathbf{p}$ gives its estimated value, $\hat{\mathbf{p}}$, at the functional minimum as seen in Eq. (4.15).

$$
\hat{\mathbf{p}}=\left(\mathbf{R}^{T} \mathbf{R}\right)^{-1} \mathbf{R}^{T} \mathbf{C}_{\text {exp }}
$$

Thus the reconstruction of the modeled aerodynamic coefficients using the estimated parameter vector is:

$$
\hat{\mathbf{C}}=\mathbf{R} \hat{\mathbf{p}}
$$

By using Eq. 4.15 the aerodynamic parameters for each coefficient can be obtained independently of one another.

\subsubsection{Accuracy Estimates}

The uncertainty of the estimate can be determined from the results assuming a normal distribution. The reconstruction of the aerodynamic coefficient has the following statistics 
relative to the experimental values. The variance of the reconstruction is obtained from Eq. (4.17) and the coefficient of determination is determined from Eq. (4.18). These metrics give a measure of the overall fit of the modeled aerodynamics.

$$
\begin{gathered}
s^{2}=\frac{\left(\mathbf{C}_{e x p}-\hat{\mathbf{C}}\right)^{T}\left(\mathbf{C}_{e x p}-\hat{\mathbf{C}}\right)}{N-n_{p}} \\
R^{2}=\frac{\hat{\mathbf{p}}^{T} \mathbf{R}^{T} \mathbf{C}_{e x p}-N \overline{\mathbf{C}}_{e x p}^{2}}{\mathbf{C}_{e x p}^{T} \mathbf{C}_{e x p}-N \overline{\mathbf{C}}_{e x p}^{2}}
\end{gathered}
$$

The uncertainty estimate of the parameters themselves are obtained from the following equations. The covariance matrix can be determined using Eq. (4.19). The standard error for each aerodynamic parameter is in the square root of the diagonal of the covariance matrix. The relative standard error, Eq. (4.21), is used to determine the significance of the parameter relative to its estimated value. This value is the same as the coefficient of variation but given as a precent.

$$
\begin{gathered}
\operatorname{Cov}(\hat{\mathbf{p}})=s^{2}\left(\mathbf{R}^{T} \mathbf{R}\right)^{-1} \\
s(\hat{\mathbf{p}})=\sqrt{\operatorname{Diag}(\operatorname{Cov}(\hat{\mathbf{p}}))} \\
R S E\left(\hat{\mathbf{p}}_{i}\right)=\frac{s\left(\hat{\mathbf{p}}_{i}\right)}{\hat{\mathbf{p}}_{i}} \times 100 \quad i=1,2, \ldots n_{p}
\end{gathered}
$$




\section{Chapter 5: Results}

The PID results of three SAGE VST data sets are presented in this chapter. The data sets consist of an unperturbed run, two perturbed runs, and both data types combined into one data set. Results were generated using the aerodynamic model and PID methods described in Chapter 4. The state variables among other variables important to the PID analysis are determined using the equations presented in the flight mechanics equations in Appendix B. An overall final set of aerodynamic parameters was chosen from the results based on the accuracy estimates and the dynamics of the SAGE model observed in the wind tunnel. This set of aerodynamic parameters was validated by comparing them to the combination of: the static wind tunnel test results, the prediction of the aerodynamic coefficients for wind tunnel runs not included in the analysis, and a simulation of steady state conditions.

\subsection{Source Data}

The wind tunnel runs used in the PID analysis were selected based on the run type (i.e. perturbed or unperturbed) because of the range of the total angle of attack and rotation rates encountered for each run type. One data set of each type was used in the analysis. The data sets consisted of wind tunnel data cropped to a certain time range to remove unwanted sections of data. For the unperturbed data this is usually a few seconds at the beginning and end of the run when the model is in a transitional phase from the tunnel speeding up at the beginning, or being rescued by pulling on the tether to avoid impacting the wall. For the perturbed data individual perturbations are cropped and combined into a single data set. The initial time is selected by observing the wind tunnel data and the video data of the run and choosing the point in which the pole used to perturb the model was removed from contact with the model. This is usually the point of highest perturbation and the model will oscillate until a steady state is reached again. The end time of the perturbation is somewhat arbitrary. It is selected once steady state is reached but may be extended until the next perturbation is initiated or the run is terminated. The lander model was usually perturbed multiple times during a single run. The perturbed and unperturbed data sets were combined into a single mixed data set in an attempt to obtain a balanced result that took both data types into account.

Smoothed data from individual wind tunnel runs were combined into a single data set by stacking the runs. This means that a data set is created by placing the data from one wind 
tunnel run immediately after the selected end of another wind tunnel run such that there is one time domain with multiple wind tunnel runs contained therein. The stacked data sets contain discontinuities but the equations of motion and the PID analysis both solve point by point with no regard for the discontinuities. In fact, the data points could be randomly scrambled and the PID analysis would yield the same solution. The main advantage of using stacked data sets is that one value can be generated for multiple wind tunnel runs. This method is used for the perturbed data set by stacking individual perturbations as well as the mixed data set.

The perturbed and unperturbed runs have different ranges of the regressors that the aerodynamic coefficients are a function of. The range of the data affects the range for which the solution is valid, beyond which is an extrapolation of the aerodynamic model. The perturbed data contains higher angles of attack and higher rotation rates, when compared to the unperturbed data, which lends itself to better estimates of the coefficients. The problem is that the time window is shorter and the lander model is in an excited state for most of the available time leading to estimates that are tailored to the specific perturbations analyzed. The unperturbed runs do not have as wide a range of wind angles and rotation rates, but they yield more accurate parameter results at the lower values of these variables. In an attempt to get the best of both data types the perturbed and unperturbed data were combined and analyzed as a mixed data set. A problem with this is that the unperturbed data may overpower the high angle estimates with the extended low angle time. The general information of the data sets used to generate the results is presented in Table 5.1 .

The actual wind tunnel runs included in each data set are as follows. The unperturbed data set consists of the data collected during Test Block 2 Run 2. This is a 60 second run that is cropped at the end to remove the rescue of the lander model due to wall encroachment. This creates a 55 second run where the lander stays at a relatively low total angle of attack where nothing of note occurs. The Perturbed data set consists of data collected during perturbations in Test Block 3 Runs 2 and 3. The two perturbations were selected as the highest quality perturbations because they have the highest initial total angle of attack, and pitch and yaw rotation rates. The first perturbation lasts approximately 15 seconds and the second only lasts about 3 seconds. The mixed data set features the runs described above stacked into a single data set. The total run time is approximately 73 seconds and the maximum total angle of attack and rotation rates are the same as the perturbed data.

Table 5.1: Wind tunnel run breakdown.

\begin{tabular}{lcccc}
\hline Run Type & Time $(\mathrm{s})$ & $\operatorname{Max} \alpha_{T}(\mathrm{deg})$ & $\operatorname{Max}|q|(\mathrm{deg} / \mathrm{s})$ & $\operatorname{Max}|r|(\mathrm{deg} / \mathrm{s})$ \\
\hline Unperturbed & 55.0 & 8.6 & 26.8 & 54.4 \\
Perturbed & 18.3 & 38.8 & 46.9 & 98.3 \\
Combined & 73.3 & 38.8 & 46.9 & 98.3 \\
\hline
\end{tabular}




\subsection{PID Results}

The following are the results for the analyzed data sets. The results are presented for the three data sets described in the previous section and a final set of parameters are selected using the best of the results from each data set.

\subsubsection{Unperturbed Results}

The results of the unperturbed data set are presented in Table 5.2. Figure 5.1 presents the regressors, reconstructions, and residuals of the axial and total normal force coefficients, and pitch and yaw moment coefficients. The total angle of attack (Figure 5.1a) stays relatively low, with only peaks above $\alpha_{T}=6^{\circ}$, which is also observable in the angles of attack and sideslip plots (Figure 5.1g). The pitch and yaw rotation rates (Figure 5.1h) also remain relatively low, in the range of $30 \mathrm{deg} / \mathrm{s}$. There are two visible oscillation modes observable in the run, a low angle oscillation followed by a larger angle oscillation which begins at about the 30 second mark. This can be seen in the total angle of attack and clock angle plots (Figures $5.1 \mathrm{a}$ and $5.1 \mathrm{~b}$ respectively) but when decomposed into angles of attack and sideslip it can be seen that the larger oscillation is primarily about the yaw axis (Figure 5.1j). The pitch axis (Figure 5.1i) does not have the same mode and stays constant through out the run. This implies that better results can be determined from the higher, more varied oscillations in the yaw plane than that of the pitch plane.

As seen in Table 5.2, the linear static aerodynamic parameters are identified with very high accuracy, usually at or below $\sim 1 \% R S E$. Due to the low maximum total angle of attack the the estimates of the $\sin ^{3} \alpha_{T}$ and damping terms $\left(C_{m_{1}}, C_{m_{2}}, C_{n_{1}}\right.$, and $\left.C_{n_{2}}\right)$ have large standard errors. The terms $C_{N_{\sin ^{3} \alpha_{T}}}$ and $C_{m_{\sin ^{3} \alpha_{T}}}$ are over estimated, upon comparison to the static wind tunnel data, in an attempt to account for nonlinearities over the small angle window. The $C_{n_{1}}$ damping term is very close to zero with a relative standard error of 93\% and therefore could be assumed zero. The roll moment coefficient has poor accuracy, $70.2 \% R S E$, and the static and damping parameters appear to have the opposite sign than would be expected. The positive damping means that the lander would possibly spin up in a simulation, overcoming the constant negative static term. With the exception of the linear static parameters the results have poor parameter estimates for the selected aerodynamic model with the unperturbed data. This data set could possibly yield better results with a linear aerodynamic model, by disregarding all squared and cubed terms, because of the lower oscillations and associated rates.

The reconstructions of the axial force coefficient (Figure 5.1c) yields a near-constant value, as expected from Eq. 4.1) and the limited total angle of attack range (Figure 5.1a). The test data shows significantly greater variation due to either noise or an un-modeled regressor. The error could be due to inadequacies in the tether model or to unsteady airflow around the irregularly shaped lander model. The reconstruction of the total normal force (Figure 5.1d) is accurate, only missing the peaks of the of the oscillations and the residual (Figure 5.1f) is evenly dispersed with a slight bias in the last 20 seconds. The reconstruction 
Table 5.2: Unperturbed PID results.

\begin{tabular}{llrlc}
\hline Coefficient & Parameter & \multicolumn{1}{c}{$\hat{\mathbf{p}}$} & $s(\hat{\mathbf{p}})$ & $R S E(\%)$ \\
\hline$C_{A}$ & $C_{A_{T}}$ & 6.6640 & $2.4 \times 10^{-3}$ & 0.04 \\
\hline \multirow{2}{*}{$C_{N_{T}}$} & $C_{N_{\sin \alpha_{T}}}$ & 3.5625 & $2.1 \times 10^{-2}$ & 0.59 \\
& $C_{N_{\sin ^{3} \alpha_{T}}}$ & 31.6751 & $2.4 \times 10^{0}$ & 7.63 \\
\hline \multirow{2}{*}{$C_{l_{o}}$} & $C_{l_{o, T}}$ & -0.0003 & $2.0 \times 10^{-4}$ & 70.20 \\
& $C_{l_{p}}$ & 0.5865 & $8.4 \times 10^{-2}$ & 14.36 \\
\hline \multirow{3}{*}{$C_{m_{o}}$} & $C_{m_{\sin \alpha_{T}}}$ & -2.0898 & $3.2 \times 10^{-2}$ & 1.52 \\
& $C_{m_{\sin 3 \alpha_{T}}}$ & 60.3896 & $4.7 \times 10^{0}$ & 7.74 \\
& $C_{m_{1}}$ & -2.7136 & $3.0 \times 10^{-1}$ & 11.12 \\
& $C_{m_{2}}$ & 308.2425 & $1.6 \times 10^{2}$ & 50.47 \\
$C_{n_{o}}$ & $C_{m_{\sin \alpha_{T}}}$ & -2.3525 & $2.5 \times 10^{-2}$ & 1.07 \\
& $C_{m_{\sin 3} \alpha_{T}}$ & 29.8734 & $2.7 \times 10^{0}$ & 9.10 \\
& $C_{n_{1}}$ & 0.2603 & $2.4 \times 10^{-1}$ & 92.95 \\
& $C_{n_{2}}$ & -648.4724 & $6.3 \times 10^{1}$ & 9.67 \\
\hline
\end{tabular}

of the pitching moment coefficient (Figure 5.1i) is generally underestimated. Low angle values are seem accurate but the higher angle values have errors. The yawing moment (Figure 5.1j) fares slightly better, the first thirty seconds of the data set contains only low angles of sideslip and it is similar to the pitching moment reconstruction. During the final 25 seconds, once the second oscillation mode is achieved, the reconstruction improves. The peaks of the reconstruction are closer but there is a slight bias in the residual (Figure 5.11).

\subsubsection{Perturbed Results}

The results of the perturbed data set are presented in Table 5.3 . Figure 5.2 presents the regressors, reconstructions, and residuals of the axial and total normal force coefficients, and pitch and yaw moment coefficients. The perturbations can be seen as the spikes in the total angle of attack plot (at zero seconds and approximately 15 seconds), a transitional phase is included after the perturbation before steady state is reached again. The total angle of attack (Figure 5.2a) oscillations are seen to damp out over the course of the first perturbation. The second perturbation is damped out quickly reaching steady state soon after the selected end. The perturbations peak at $\alpha_{T} \simeq 40^{\circ}$ and there is more data above the $8^{\circ}$ maximum of the unperturbed data. There is an observable dead area immediately following the first perturbation between 2.5 and 5 seconds. This likely marks the transition from perturbed to steady state. The damping of the large scale oscillations is still in control and the small angle instabilities have not yet taken effect. As with the unperturbed data, the yawing plane seems to have a majority of the oscillations and will therefor yield better 
results than those of the pitching moment.

The linear static aerodynamics are estimated with less accuracy, by observing the $R S E$ values in Table 5.3, than that of the unperturbed data set. This can be attributed to the length of the run, as there are fewer data points than in the unperturbed data set. The nonlinear terms are estimated with higher accuracy, a lower $R S E$, than in the unperturbed data. The issues with over estimating the $C_{N_{\sin ^{3} \alpha_{T}}}$ and $C_{m_{\sin ^{3} \alpha_{T}}}$ terms are resolved due to the larger number of data points at higher angles of attack. The $C_{N_{\sin ^{3} \alpha_{T}}}$ term has a higher relative standard error of $14.6 \%$, compared to $7.6 \%$ for unperturbed, but the estimated value of the parameter is much more believable despite having a larger relative standard error. The roll moment coefficient is estimated with better accuracy, by observing the $R S E$ values in Table 5.3, than with the unperturbed data and with the expected sign. The parameters for the pitching moment coefficient have lower relative standard errors than those for the yawing moment coefficient. Overall the results are improved from those of the unperturbed data.

The axial force coefficient reconstruction (Figure 5.2c) matches well at the peaks of the perturbations but the experimental value varies at lower total angles of attack. This same behavior was observed in the unperturbed results. There appears to be two modes present in the experimental axial force coefficient: a modeled mode that covers higher total angle of attack perturbations, and an un-modeled mode that is present at low total angle of attack during steady state. The normal force coefficient reconstruction (Figure 5.2d) matches well during the perturbations with smaller differences in the sections approaching steady state. The reconstruction of the pitching moment coefficient (Figure 5.2i) has a poor fit to the

Table 5.3: Perturbed PID results.

\begin{tabular}{llrcc}
\hline Coefficient & Parameter & \multicolumn{1}{c}{$\hat{\mathbf{p}}$} & $s(\hat{\mathbf{p}})$ & $R S E(\%)$ \\
\hline$C_{A}$ & $C_{A_{T}}$ & 6.6557 & $6.8 \times 10^{-3}$ & 0.10 \\
\hline \multirow{2}{*}{$C_{N_{T}}$} & $C_{N_{\sin \alpha_{T}}}$ & 3.0643 & $1.5 \times 10^{-2}$ & 0.48 \\
& $C_{N_{\sin ^{3} \alpha_{T}}}$ & -0.4802 & $7.0 \times 10^{-2}$ & 14.61 \\
\hline \multirow{2}{*}{$C_{l_{o}}$} & $C_{l_{o, T}}$ & 0.0125 & $9.0 \times 10^{-4}$ & 7.44 \\
& $C_{l_{p}}$ & -5.6816 & $2.8 \times 10^{-1}$ & 4.91 \\
\hline \multirow{3}{*}{$C_{m_{o}}$} & $C_{m_{\sin \alpha_{T}}}$ & -1.0213 & $2.2 \times 10^{-2}$ & 2.16 \\
& $C_{m_{\sin 3} \alpha_{T}}$ & 0.7685 & $2.8 \times 10^{-1}$ & 36.94 \\
& $C_{m_{1}}$ & -5.1048 & $4.1 \times 10^{-1}$ & 7.93 \\
& $C_{m_{2}}$ & -89.2280 & $3.2 \times 10^{1}$ & 35.34 \\
\hline$C_{n_{o}}$ & $C_{m_{\sin \alpha_{T}}}$ & -1.9508 & $3.0 \times 10^{-2}$ & 1.56 \\
& $C_{m_{\sin 3} \alpha_{T}}$ & 2.4853 & $1.5 \times 10^{-1}$ & 5.84 \\
& $C_{n_{1}}$ & -7.4801 & $3.7 \times 10^{-1}$ & 4.98 \\
& $C_{n_{2}}$ & -162.7648 & $7.1 \times 10^{0}$ & 4.39 \\
\hline
\end{tabular}


experimental values but most of the oscillations are in the yaw plane. The reconstruction of the yawing moment coefficient (Figure 5.2j), like the normal force coefficient, fits best in the perturbed section of the data with larger errors in the sections approaching steady state. Similar to the unperturbed data there is a slight bias in the steady state section of the reconstruction as seen in the residuals (Figure 5.21).

\subsubsection{Combined Results}

The results of the combined data set are presented in Table 5.4. Figure 5.3 presents the regressors, reconstructions, and residuals of the axial and total normal force coefficients, and pitch and yaw moment coefficients. This data set is a concatenation of the perturbed and unperturbed data sets. The regressors are thus the same as described in the previous sections. This is to give results that lie between the two extremes. Note that the amount of time for the data type plays into the values obtained in the PID analysis. If less time of the unperturbed data set is used then the results will be closer to the perturbed results, the opposite is also true. For this analysis the full time of both data sets is used. resulting in 72 seconds of data with two perturbations.

The overall effect of analyzing both runs is that the aerodynamic parameters have the accuracy of the unperturbed data set but the more appropriate values at high angles of attack seen in the perturbed data set, see the results in Table 5.4. This is true for the static forces; all parameters having the same or better relative standard error than with

Table 5.4: Perturbed and unperturbed PID results.

\begin{tabular}{llrcc}
\hline Coefficient & Parameter & \multicolumn{1}{c}{$\hat{\mathbf{p}}$} & $s(\hat{\mathbf{p}})$ & $R S E(\%)$ \\
\hline$C_{A}$ & $C_{A_{T}}$ & 6.6620 & $2.5 \times 10^{-3}$ & 0.04 \\
\hline \multirow{2}{*}{$C_{N_{T}}$} & $C_{N_{\sin \alpha_{T}}}$ & 3.5129 & $9.8 \times 10^{-3}$ & 0.28 \\
& $C_{N_{\sin \alpha_{T}}}$ & -2.0035 & $6.1 \times 10^{-2}$ & 3.03 \\
\hline \multirow{2}{*}{$C_{l_{o}}$} & $C_{l_{o, T}}$ & 0.0023 & $3.0 \times 10^{-4}$ & 11.98 \\
& $C_{l_{p}}$ & -1.1080 & $9.4 \times 10^{-2}$ & 8.49 \\
\hline \multirow{4}{*}{$C_{m_{o}}$} & $C_{m_{\sin \alpha_{T}}}$ & -1.3572 & $1.3 \times 10^{-2}$ & 0.96 \\
& $C_{m_{\sin \alpha_{T}}}$ & 2.1824 & $2.2 \times 10^{-1}$ & 10.03 \\
& $C_{m_{1}}$ & -3.6522 & $2.2 \times 10^{-1}$ & 6.07 \\
& $C_{m_{2}}$ & -72.4599 & $2.4 \times 10^{1}$ & 33.77 \\
\hline \multirow{4}{*}{$C_{n_{o}}$} & $C_{m_{\sin \alpha_{T}}}$ & -2.0655 & $1.3 \times 10^{-2}$ & 0.64 \\
& $C_{m_{\sin \alpha_{T}}}$ & 2.5278 & $1.0 \times 10^{-1}$ & 4.12 \\
& $C_{n_{1}}$ & -3.3025 & $1.8 \times 10^{-1}$ & 5.40 \\
& $C_{n_{2}}$ & -205.4289 & $5.2 \times 10^{0}$ & 2.55 \\
\hline
\end{tabular}


the data sets analyzed individually. The nonlinear $C_{N_{\sin ^{3} \alpha_{T}}}$ achieves lower relative standard error than in either of the source data sets. The roll moment coefficient parameters are less accurate because the unperturbed data yielded incorrect values for the parameters meaning that the perturbed data set gives results more inline with what would be expected. The yawing moment coefficient parameters are estimated with high accuracy, higher than either of the source data sets. The pitching moment is not as accurate due to the majority of the oscillations being in the yaw plane.

The quality of the reconstructions of the coefficients doesn't change much as compared to the unperturbed and perturbed data sets. The axial force coefficient reconstruction (Figure 5.3c fits in a similar manner but has a slightly higher standard error. The normal force coefficient (Figure 5.3d) has a similar reconstruction as the other data sets but retains approximately the same standard error. The bias of the residual (Figure 5.3f) present in the latter half of the unperturbed data set and in the steady state sections of the perturbed data set are still there but are not as noticeable. The pitching moment coefficient reconstruction (Figure 5.3i) is quite poor but consistent with the results from the other data sets. The yawing moment coefficient reconstruction (Figure 5.3j) is fair with a smaller standard error than the perturbed data, closer to the unperturbed. The overall results from the combined data set are superior to the results of either data set individually.

\subsubsection{Selected Parameters}

Taking the three sets of aerodynamic parameters into account a final set of parameters was chosen that will best represent the aerodynamics of the SAGE lander. This final set of aerodynamic parameters is presented in Table 5.5. The axial force coefficient parameter was chosen from the combined data set because of the high accuracy and the quality of the reconstructed data at large angles of attack. The normal force parameters are also selected

Table 5.5: Selected aerodynamic parameter set.

\begin{tabular}{lllrc}
\hline Coefficient & Data Set & Parameter & $\hat{\mathbf{p}}$ & $s(\hat{\mathbf{p}})$ \\
\hline$C_{A}$ & Combined & $C_{A_{T}}$ & 6.6620 & $2.5 \times 10^{-3}$ \\
\hline \multirow{2}{*}{$C_{N_{T}}$} & \multirow{2}{*}{ Combined } & $C_{N_{\sin \alpha_{T}}}$ & 3.5129 & $9.8 \times 10^{-3}$ \\
& & $C_{N_{\sin ^{3} \alpha_{T}}}$ & -2.0035 & $6.1 \times 10^{-2}$ \\
\hline \multirow{2}{*}{$C_{l_{o}}$} & \multirow{2}{*}{ Perturbed } & $C_{l_{o, T}}$ & 0.0125 & $9.0 \times 10^{-4}$ \\
& & $C_{l_{p}}$ & -5.6816 & $2.8 \times 10^{-1}$ \\
\hline \multirow{3}{*}{$C_{m_{o}}$ and $C_{n_{o}}$} & \multirow{2}{*}{ Combined } & $C_{m_{\sin \alpha_{T}}}$ & -2.0655 & $1.3 \times 10^{-2}$ \\
& & $C_{m_{\sin ^{3} \alpha_{T}}}$ & 2.5278 & $1.1 \times 10^{-1}$ \\
& & $C_{m_{1}}=C_{n_{1}}$ & -3.3025 & $1.8 \times 10^{-1}$ \\
& & $C_{m_{2}}=C_{n_{2}}$ & -205.4289 & $5.2 \times 10^{0}$ \\
\hline
\end{tabular}


from the combined data set because they are the best results of all three data sets. The roll moment coefficient parameters were chosen from the perturbed data set because it offers the highest roll rates and gives results that are more inline with a priori knowledge (i.e., having negative damping). The pitch and yaw moment coefficient parameters are selected from the combined data set because it gives the best accuracy and the best estimates for the nonlinear cubic terms at larger angles of attack. The pitch and yaw parameters were selected to be equal due to the near axisymmetric shape of the lander.

\subsection{Validation}

The following methods were used to validate the results. The static wind tunnel data is compared to the results for consistency. A comparison of the reconstructions of the coefficients using data that was not used in the PID analysis is conducted. Steady state values achieved in flight simulations using the parameters are compared with those observed in dynamic wind tunnel test data.

\subsubsection{Static Comparison}

By comparing the estimated static parameters to the results of the static wind tunnel testing it is possible to validate the static portion of the aerodynamic coefficients. Figure 5.4 shows the results of the static wind tunnel test and the functions estimated from the PID analysis. The experimental data is a model that assumes axisymmetry and combines the values for all roll angles. This is the same way that the aerodynamic model for the PID analysis works, so it is an apples to apples comparison. The axial and normal force coefficients, and the static portion of the pitch and yaw coefficient are plotted against the total angle of attack. The axial force coefficient curve has a slight difference at total angles of attack below $\sim 5^{\circ}$ but the fit over the rest of the angle range is good. The normal force coefficient is slightly over estimated at high total angles of attack but fits well below $\sim 10^{\circ}$. The same can be said about the pitching and yawing moment coefficient which fits well in the initial linear section to $\sim 15^{\circ}$ at which point there is a change in slope and a second linear section. In the second linear section the modeled coefficient is under estimated although the function is curving upwards to become consistent the static data. Overall, the estimated static aerodynamic models match very well to the static wind tunnel data thus validating the static aerodynamic parameters.

\subsubsection{Predictive Comparison}

The selected set of aerodynamic parameters was used to reconstruct the aerodynamic coefficients of a run not used in the PID analyses. The purpose of doing this was to evaluate the generality and applicability of the aerodynamic parameters identified. Figure 5.6 shows the reconstruction of one of the perturbations in Test Block 3 Run 3. The perturbation has a moderate maximum total angle of attack of approximately $18^{\circ}$ which quickly dies out, 
consistent with the other perturbed data sets. Overall, the reconstruction quality is comparable with that of the reconstruction of the source data set in Figure 5.3. Areas where the reconstruction falls short include: the $C_{A}$ reconstruction, and the bias in the $C_{n_{o}}$ reconstruction; seen in Figures $5.6 \mathrm{c}$ and 5.6j] respectively. The standard error on the $C_{A}$ coefficient is almost twice as large as that seen in the source data set and there is an associated bias in the residual. The yaw moment coefficient has a larger standard error and a significant bias. The bias is consistent with those seen in the perturbations in the source data set. The total normal force and pitching moment have similar characteristics as the fit to the source data set with comparable standard errors and little bias to the residuals.

\subsubsection{Simulation Comparison}

The model behavior during Test Block 2 Run 2 (unperturbed) was simulated using the SAGE aerodynamic model, as described in section 4.1.2, which incorporated the selected aerodynamic parameters in Table 5.5. The unperturbed run was selected because it represents the lander's flight dynamics in quasi-steady state flight. The results of this simulation were compared against the wind tunnel test data to evaluate the validity of the aerodynamic models, parameter identification, and the simulation. The initial conditions and tunnel wind velocity time history for the simulated run was used so as to yield data that would allow for a valid comparison. The simulation used for this analysis has been independently verified and validated for correctness and was also used to verify the correctness of the PID code (see Appendix C). The off-vertical angle, $\Theta$, (see Figure 5.7) and the pitch and yaw rotation rate, $\omega_{q, r}$, were used as the primary comparison between the data sets. The two values are determined by Eqs. (5.1) and (5.2). These values give insight to both the static and dynamic portions of the oscillations of the model lander. The metrics were also used by the SAGE proposal team to evaluate possible landing impact angles and oscillation rates for imaging.

$$
\begin{gathered}
\Theta=\arccos \left(\cos \left(R_{y}\right) \cos \left(R_{z}\right)\right) \\
\omega_{q, r}=\sqrt{q^{2}+r^{2}}
\end{gathered}
$$

Table 5.6: Comparison of test data against simulation results.

\begin{tabular}{llrl}
\hline & & Test & Simulation \\
\hline \multirow{3}{*}{$(\mathrm{deg})$} & Mean & 2.5653 & 1.3257 \\
& Median & 2.3575 & 1.2761 \\
& Std Err & 1.4957 & 0.2028 \\
\hline \multirow{3}{*}{$\omega_{q, r}(\mathrm{deg} / \mathrm{s})$} & Mean & 14.8574 & 1.3210 \\
& Median & 12.3386 & 0.2489 \\
& Std Err & 9.8990 & 2.3376 \\
\hline
\end{tabular}


Figure 5.8 shows the results of this analysis. By examining the time histories of $\Theta$ and $\omega_{q, r}$, it can be determined that the simulation does not yield a good representation of the experimental data. There is significant damping in the aerodynamic model that removes the oscillation caused by the initial conditions. The SAGE lander model has low total angle of attack instabilities that are not captured by the current model. Histograms yield a better comparison to the steady state behavior than a time history comparison (Figures $5.8 \mathrm{c}$ and $5.8 \mathrm{~d}$ ). The mean, median, and standard error were determined for $\Theta$ and $\omega_{q, r}$ in both the experimental and simulated data. These values are presented in Table 5.6. The $\Theta$ angle settles on a mean steady state value of approximately $2.5^{\circ}$ for the test data and approximately $1.3^{\circ}$ for the simulation. The value for the data is almost twice as large for the simulation. The $\omega_{q, r}$ rotation rate has a mean value of approximately $14.9^{\circ} / \mathrm{s}$ where as in the simulation all rotation is damped out so there is no, or very little, pitch or yaw rotations. These results imply that there is a low angle instability to the lander which is not captured by the simulation. 


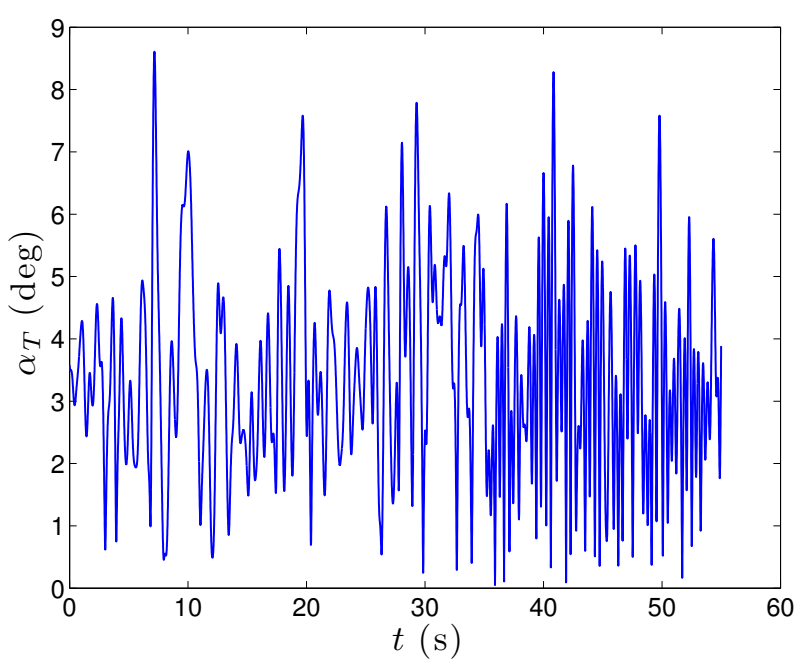

(a) Total angle of attack.

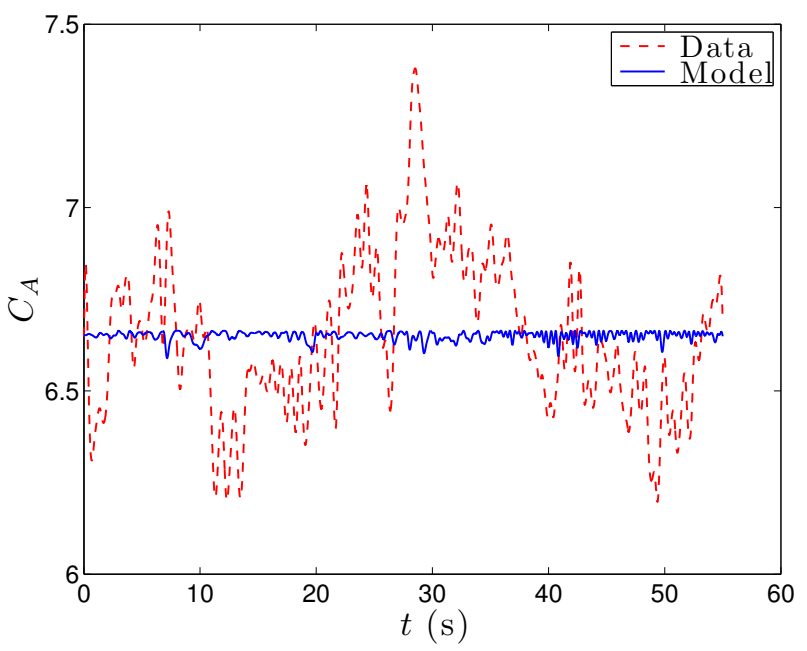

(c) Axial force coefficient.

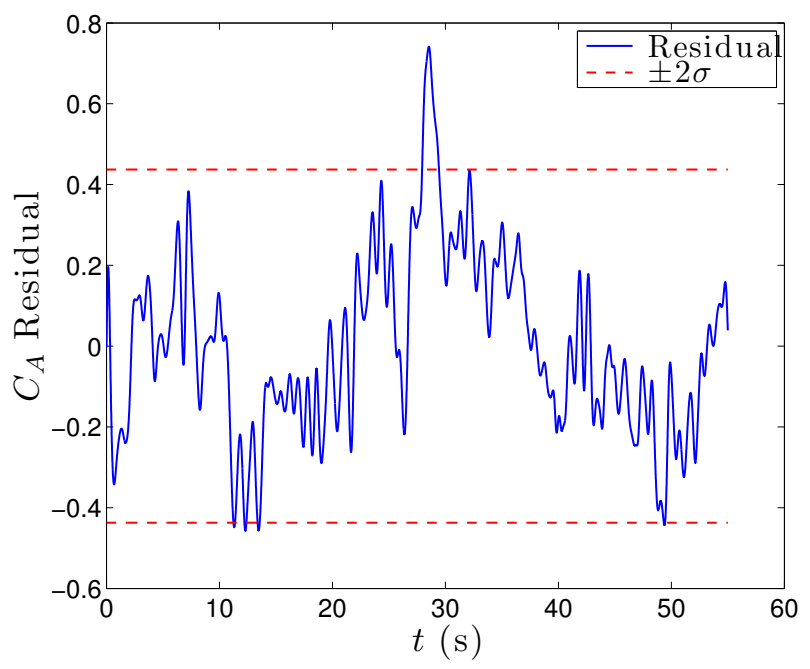

(e) Axial force coefficient residual.

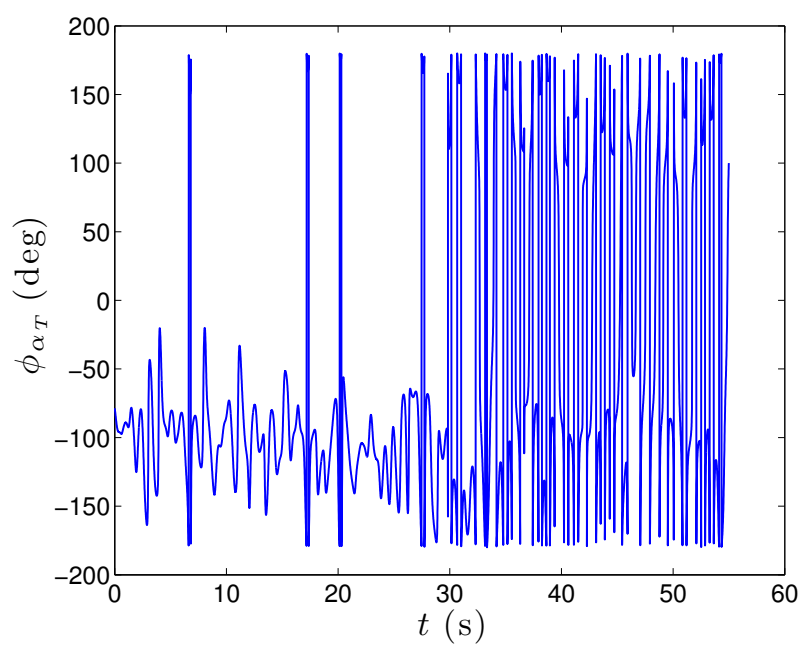

(b) Total angle of attack clock angle.

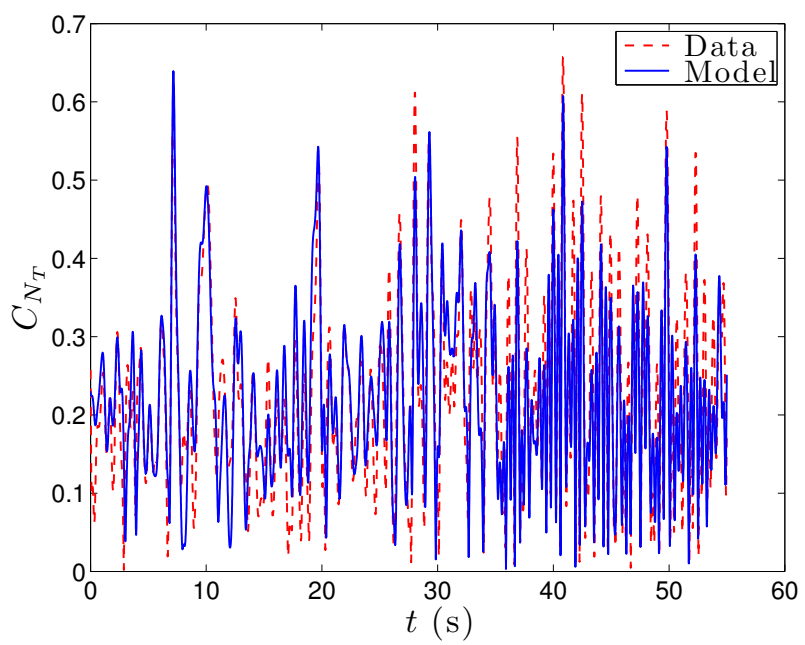

(d) Normal force coefficient.

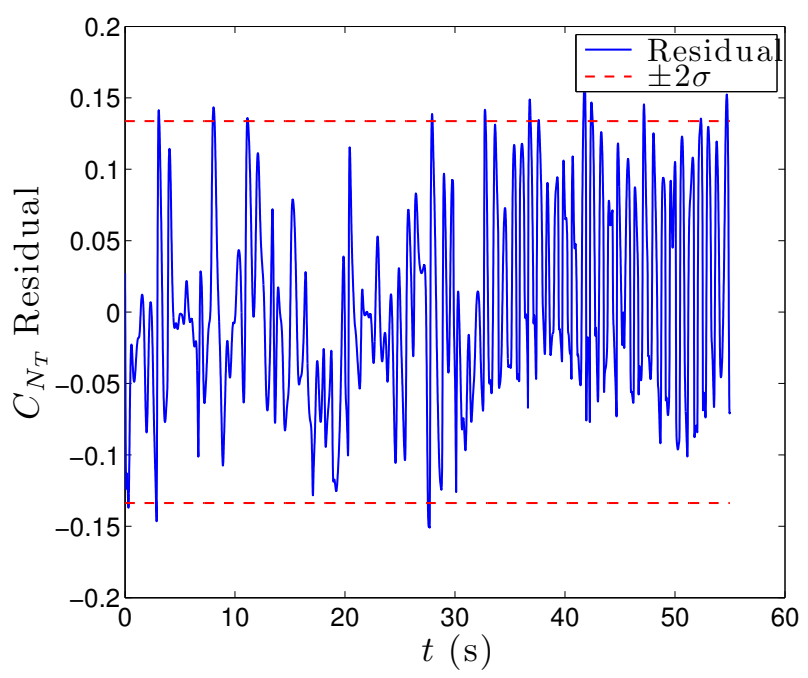

(f) Normal force coefficient residual.

Figure 5.1: Unperturbed results. 


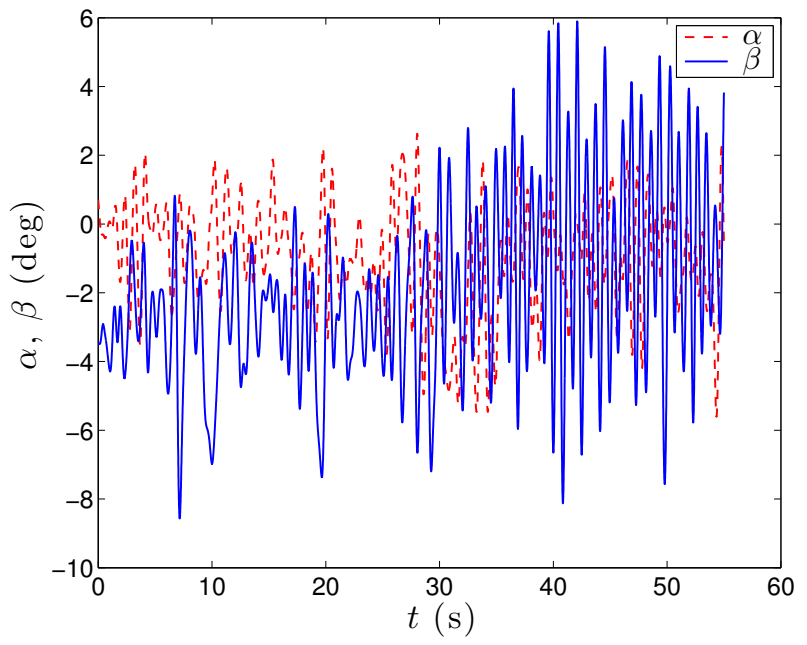

(g) Angles of attack and side slip.

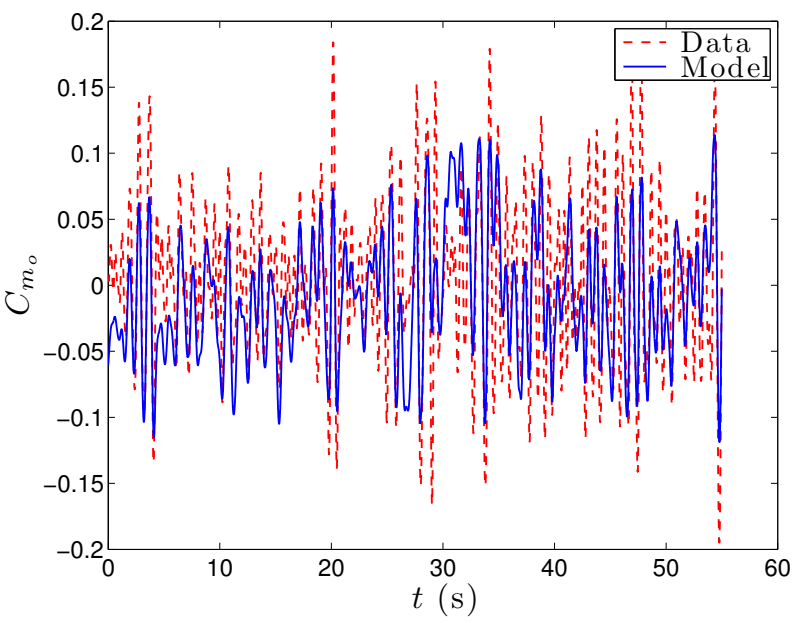

(i) Pitch moment coefficient.

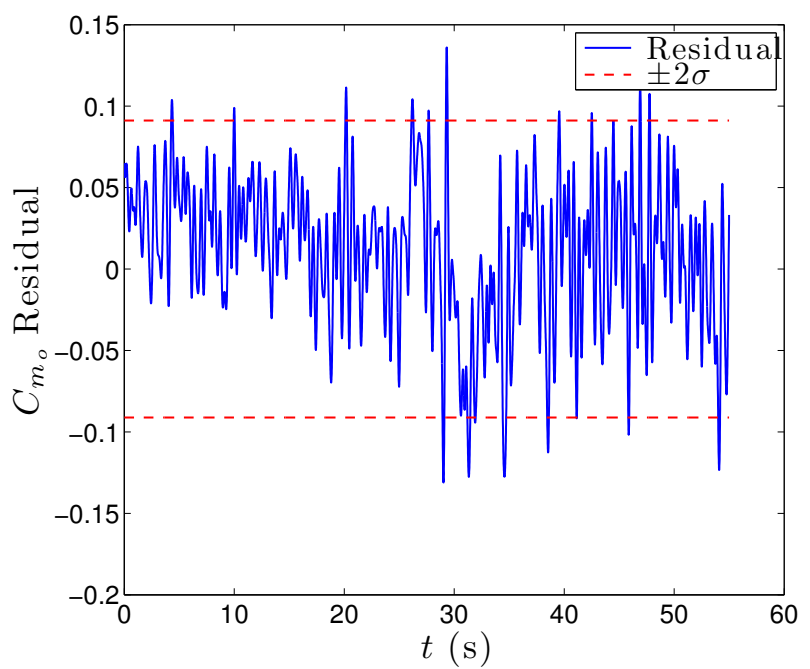

(k) Pitch moment coefficient residual.

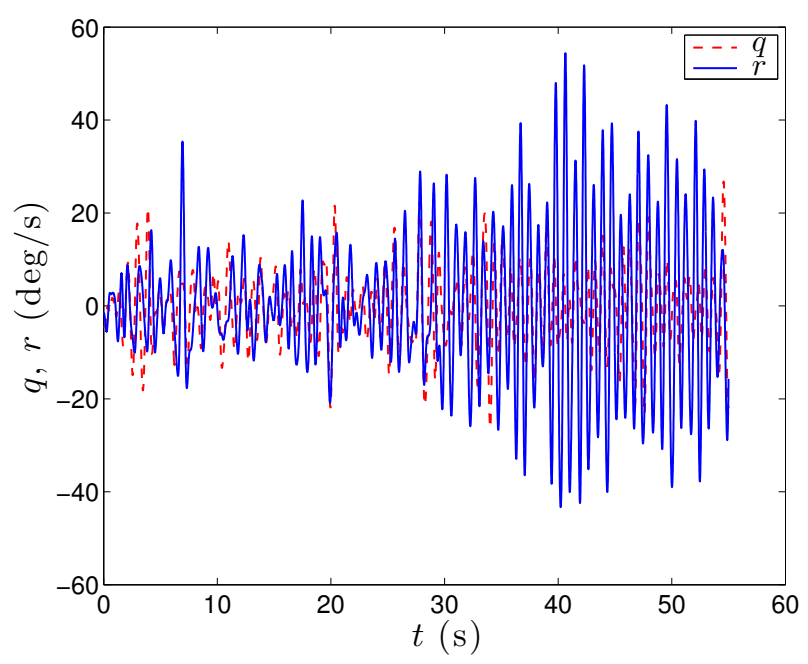

(h) Pitch and yaw rates.

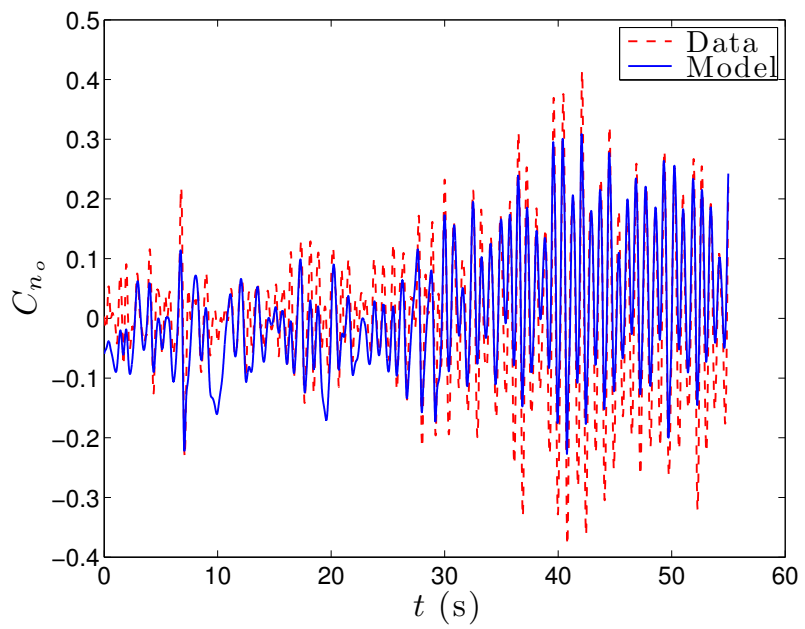

(j) Yaw moment coefficient.

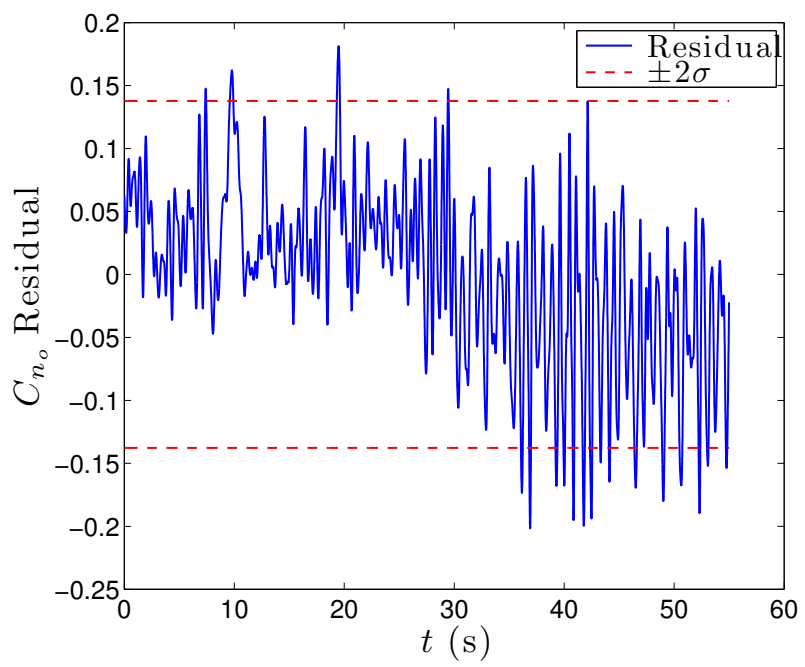

(l) Yaw moment coefficient residual.

Figure 5.1: Unperturbed results. 


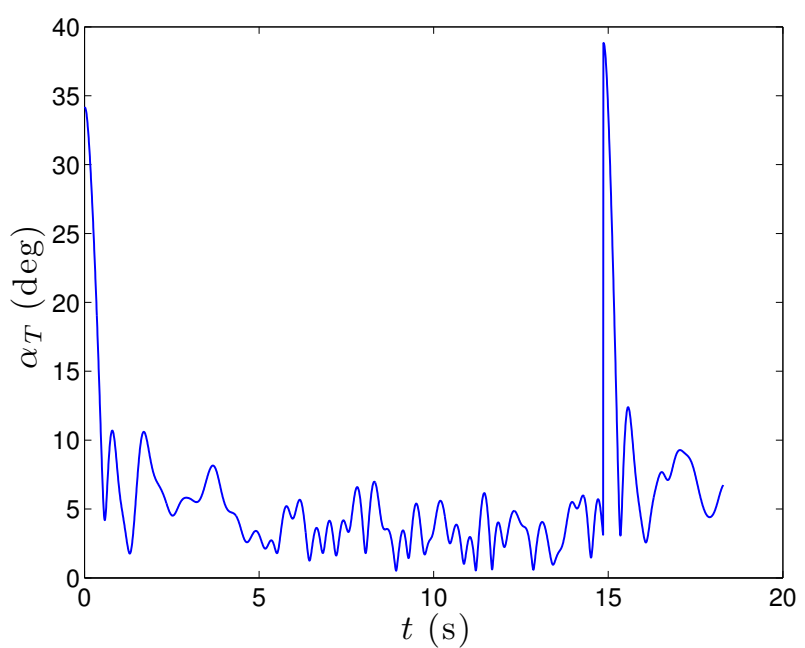

(a) Total angle of attack.

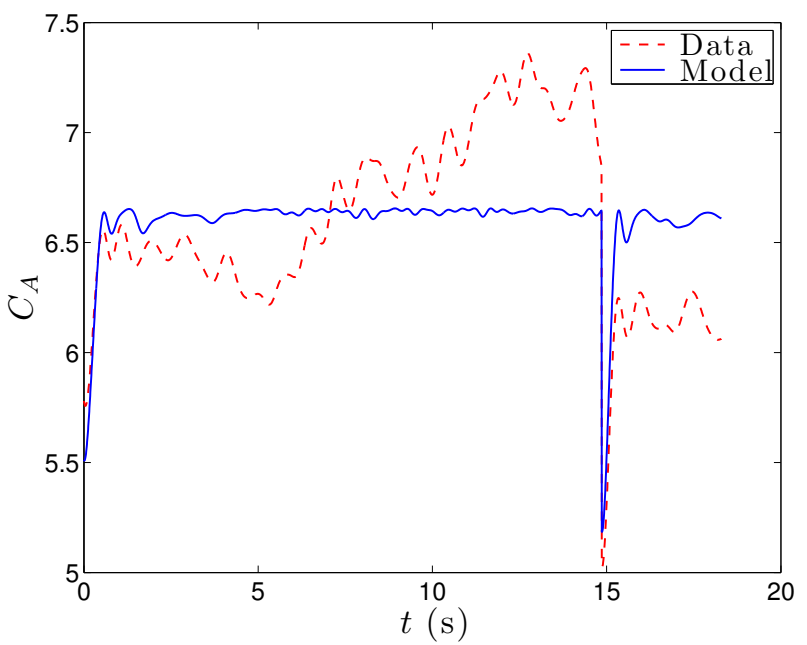

(c) Axial force coefficient.

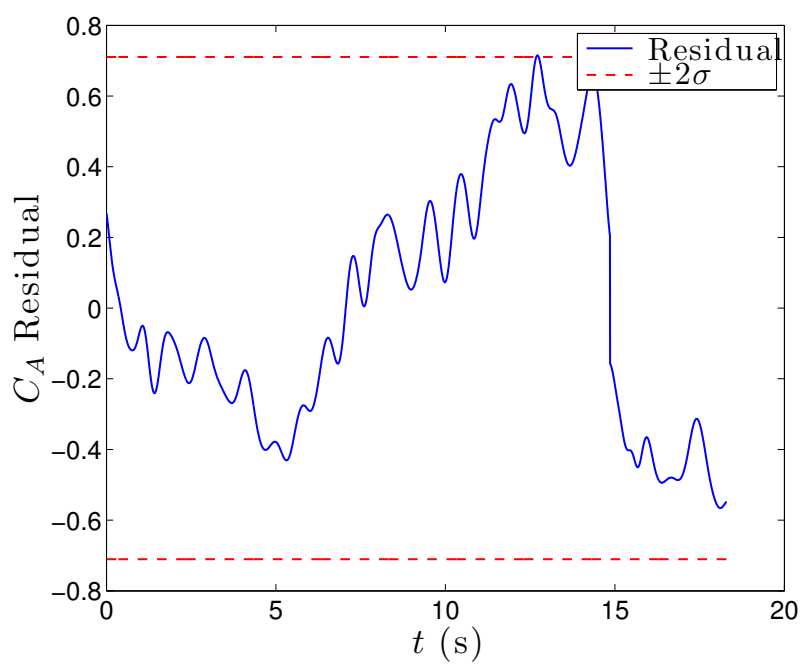

(e) Axial force coefficient residual.

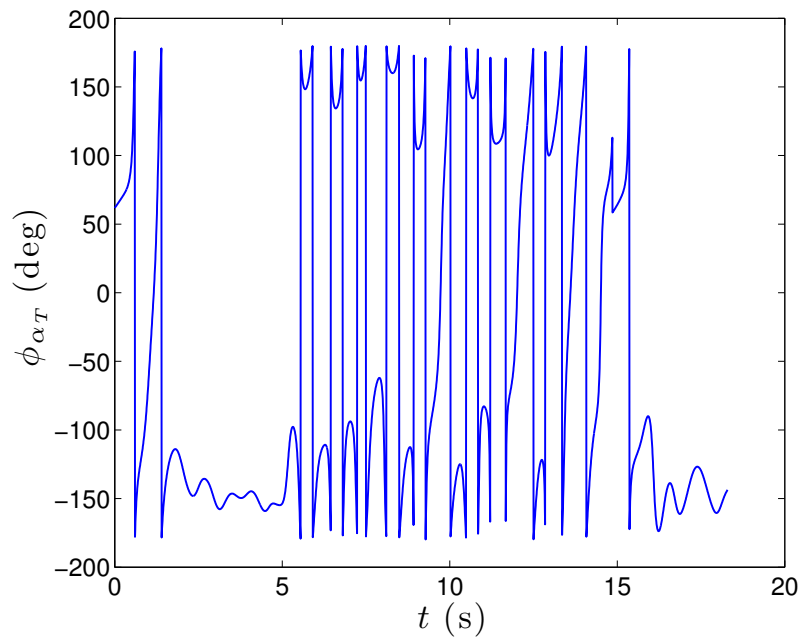

(b) Total angle of attack clock angle.

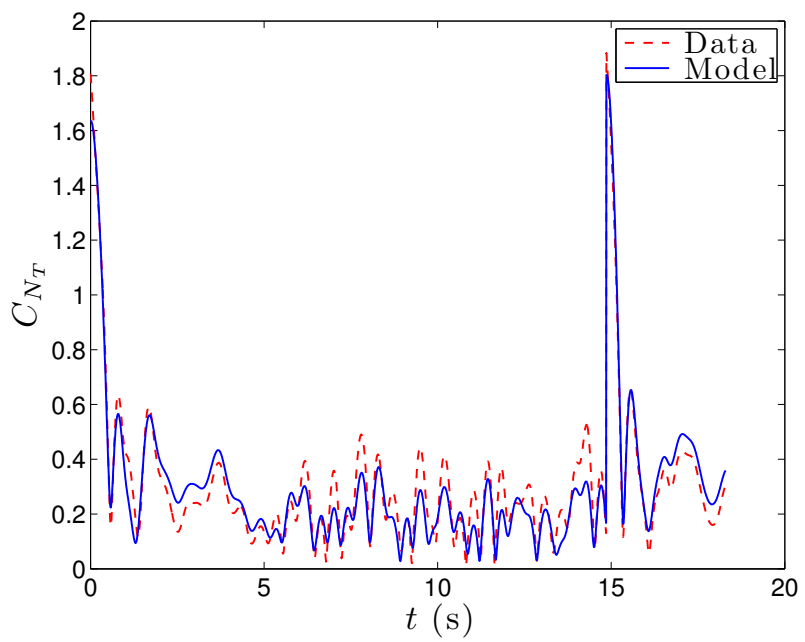

(d) Normal force coefficient.

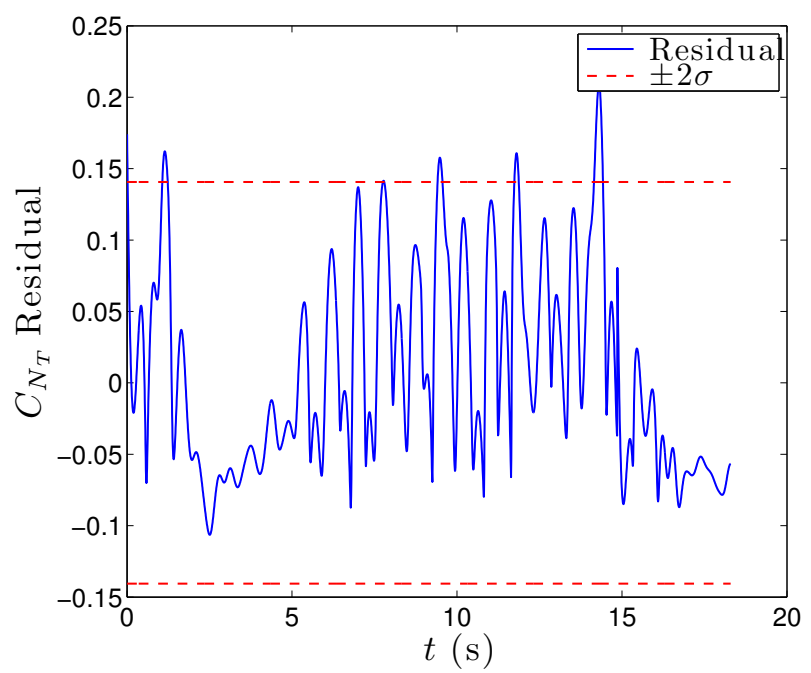

(f) Normal force coefficient residual.

Figure 5.2: Perturbed results. 


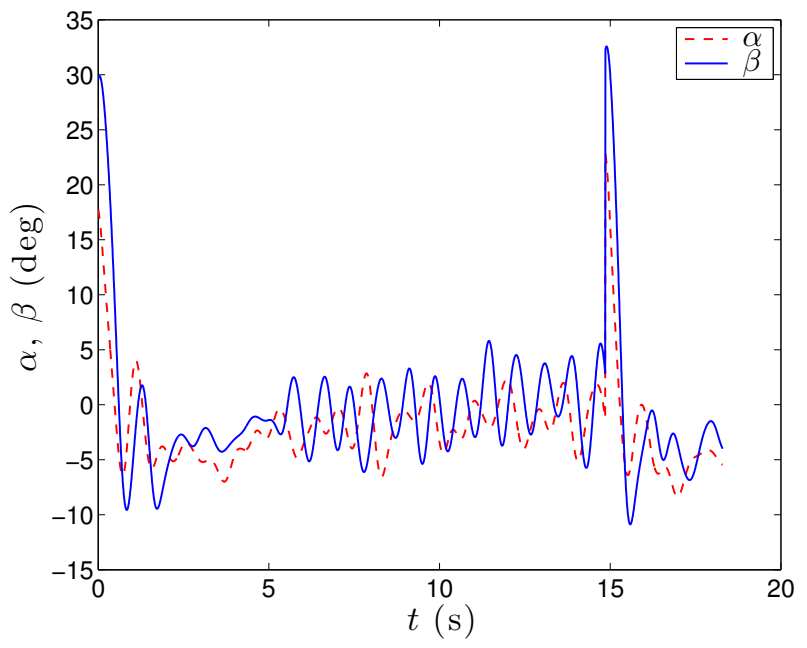

(g) $\alpha$ and $\beta$ angles.

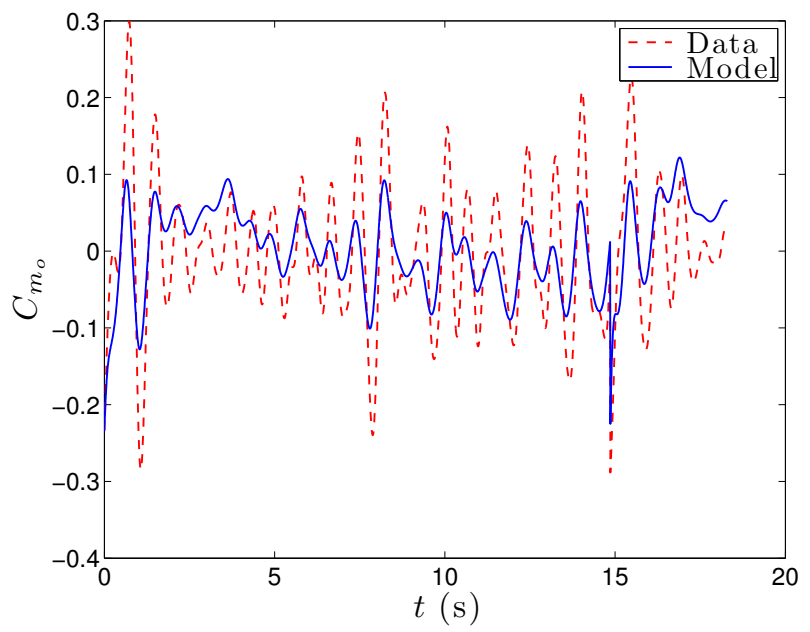

(i) Pitch moment coefficient.

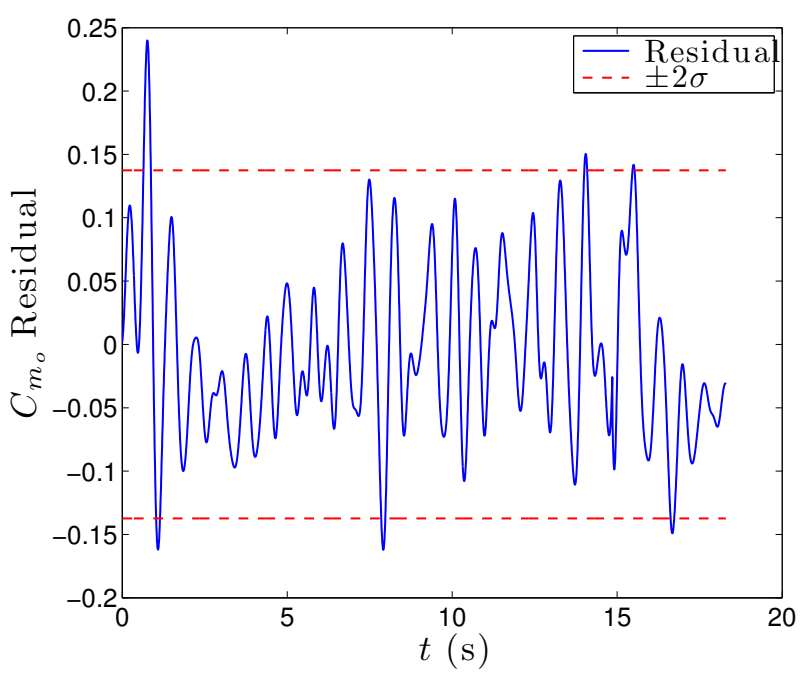

(k) Pitch moment coefficient residual.

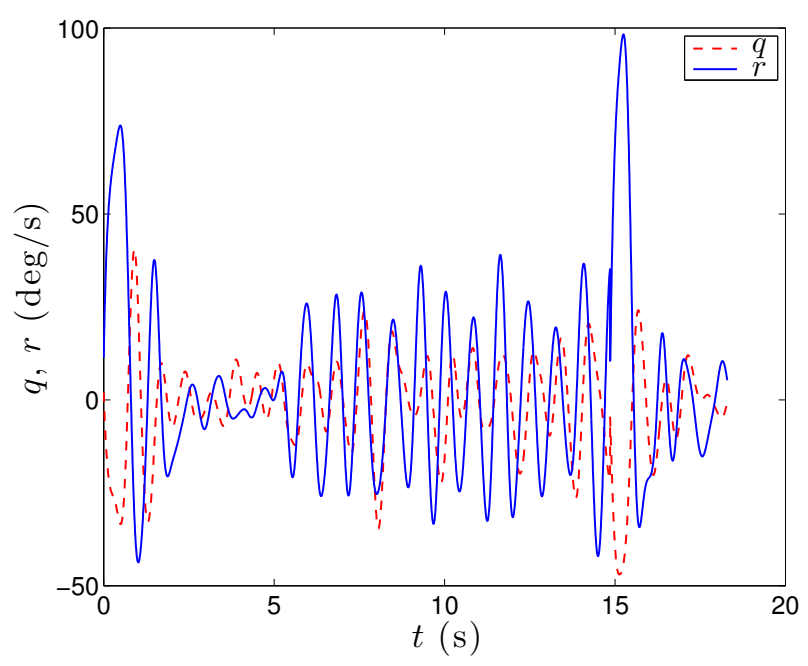

(h) $q$ and $r$ rotation rates.

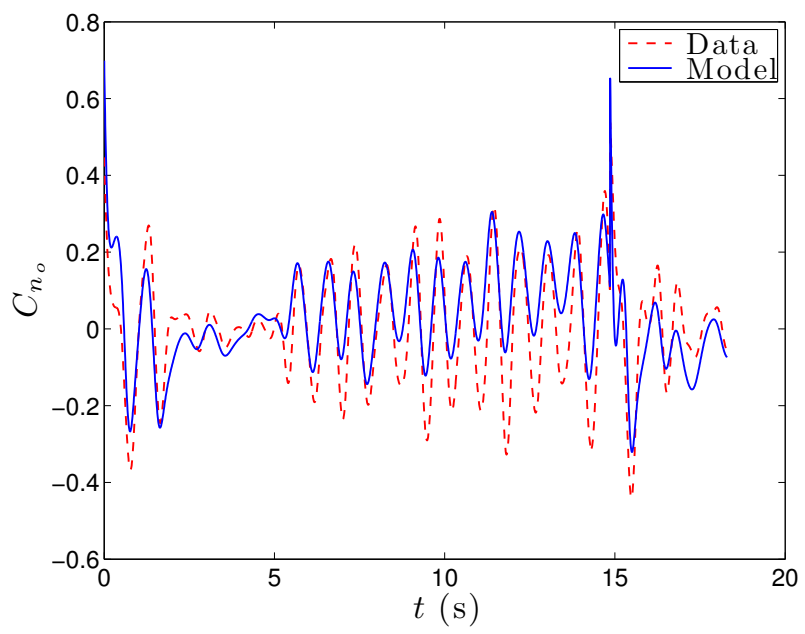

(j) Yaw moment coefficient.

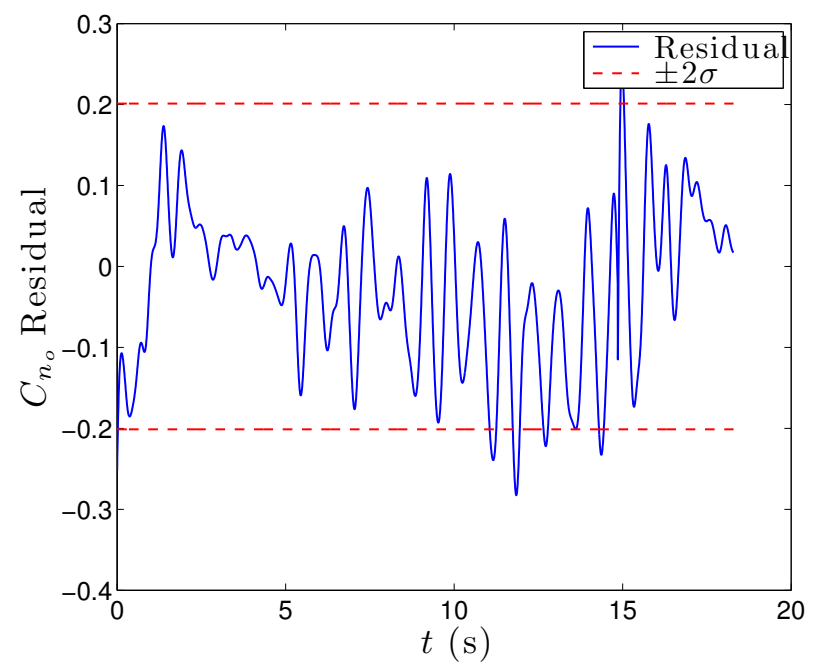

(l) Yaw moment coefficient residual.

Figure 5.2: Perturbed results. 


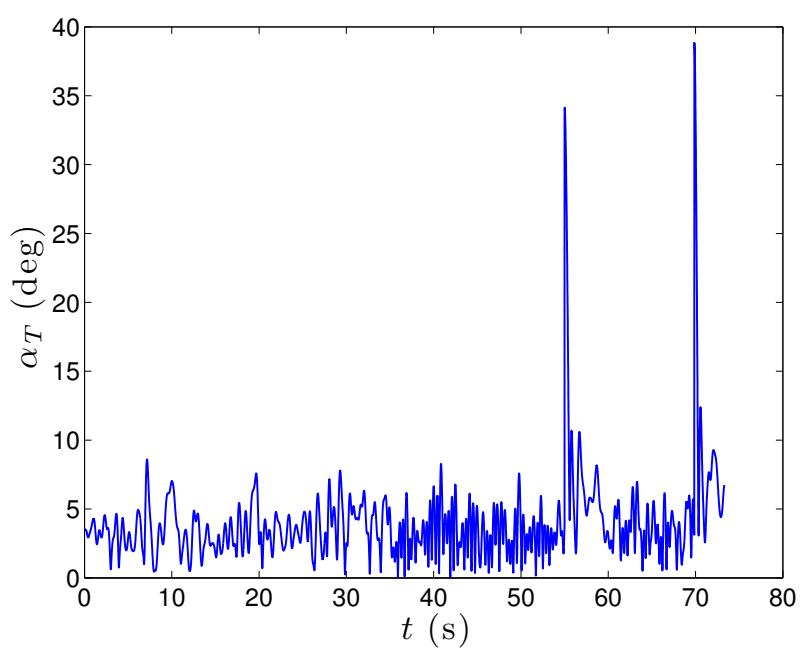

(a) Total angle of attack.

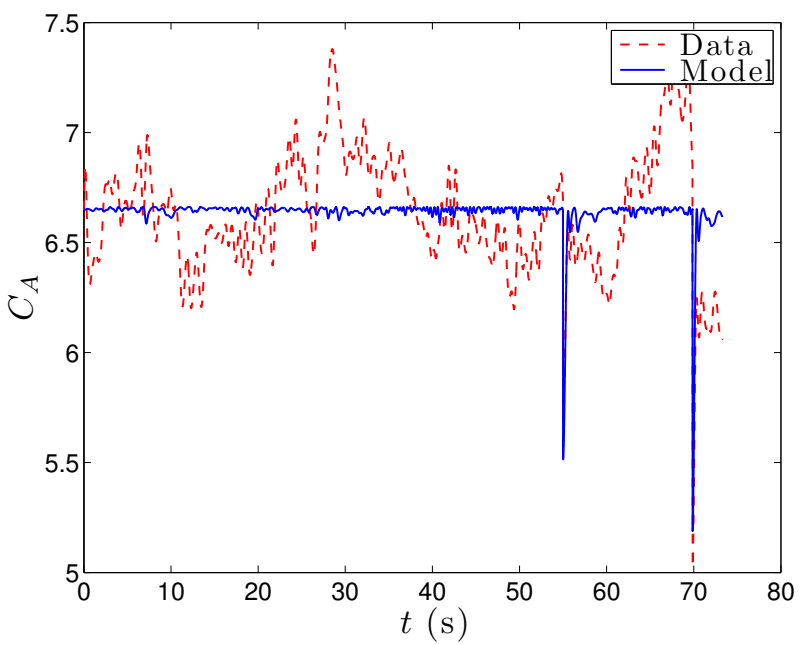

(c) Axial force coefficient.

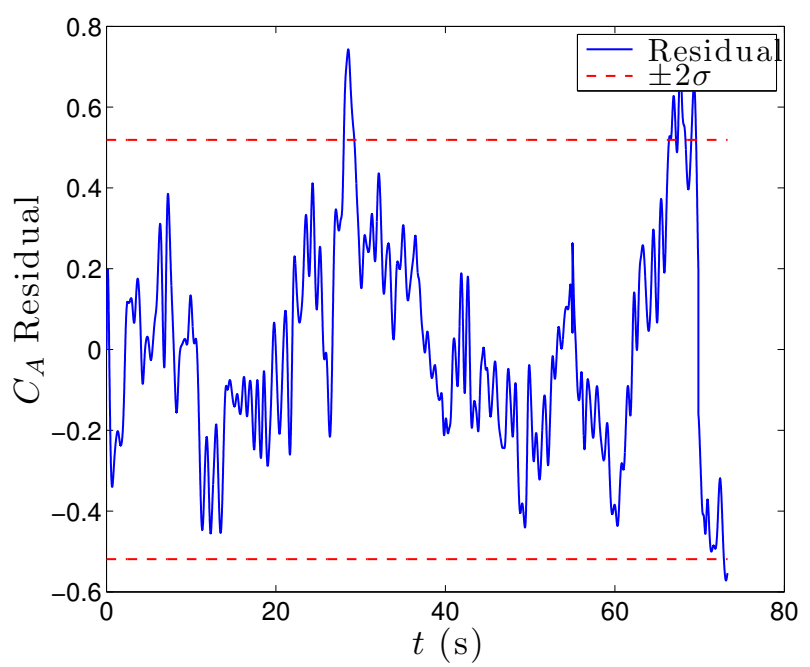

(e) Axial force coefficient residual.

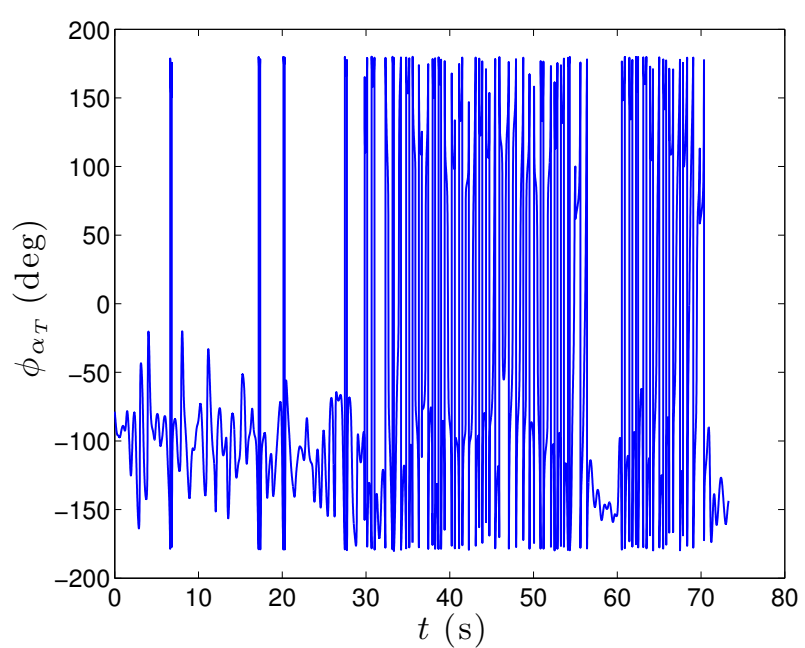

(b) Total angle of attack clock angle.

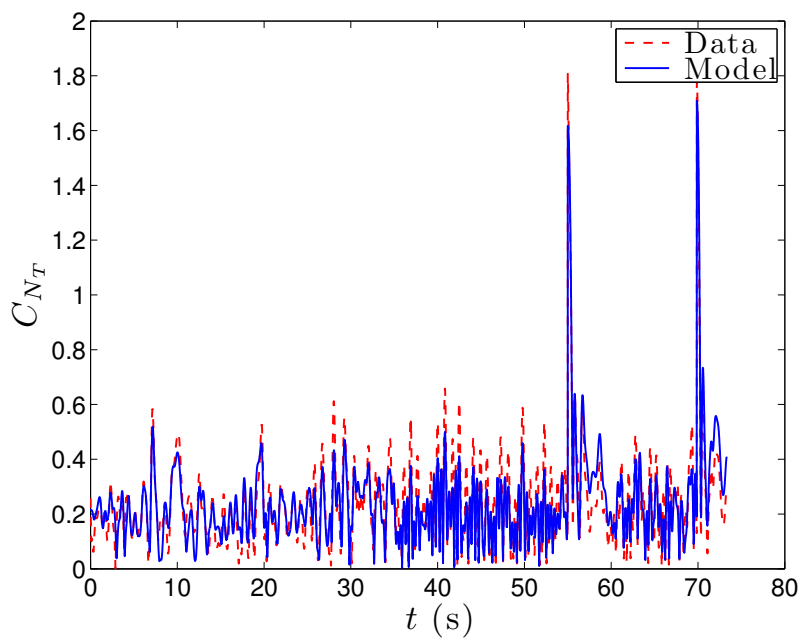

(d) Normal force coefficient.

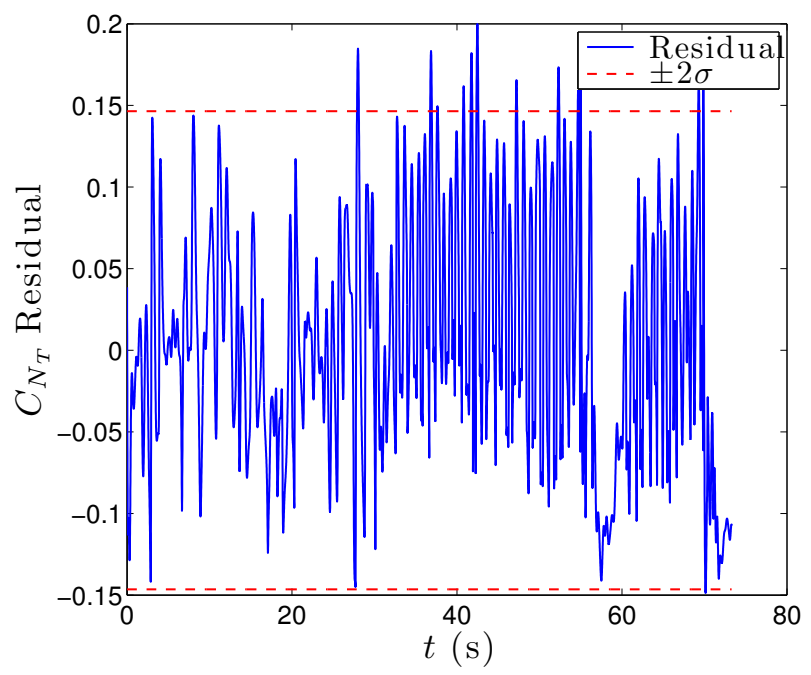

(f) Normal force coefficient residual.

Figure 5.3: Combined results. 


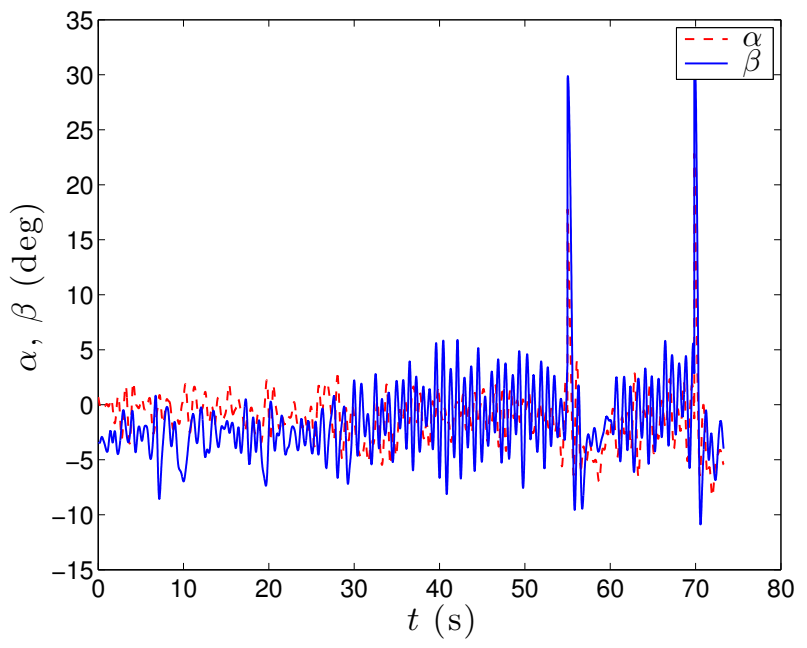

(g) $\alpha$ and $\beta$ angles.

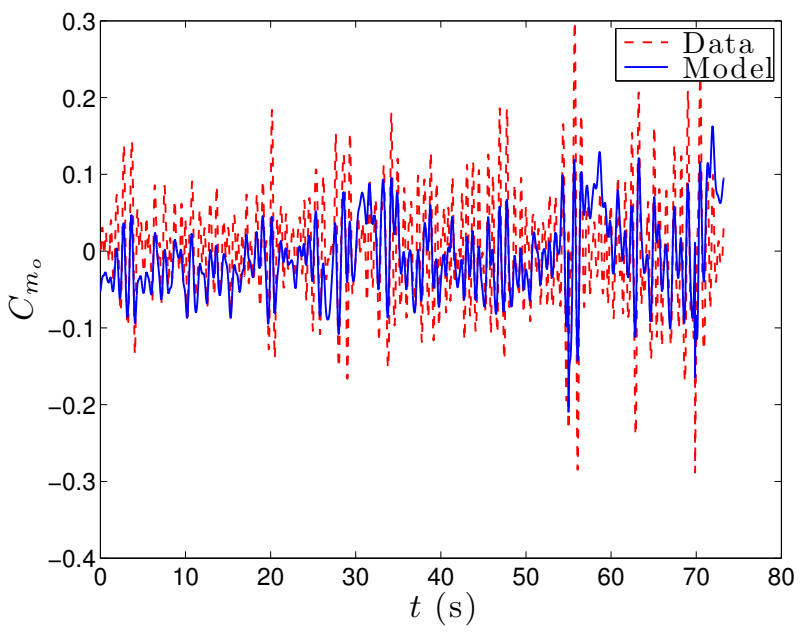

(i) Pitch moment coefficient.

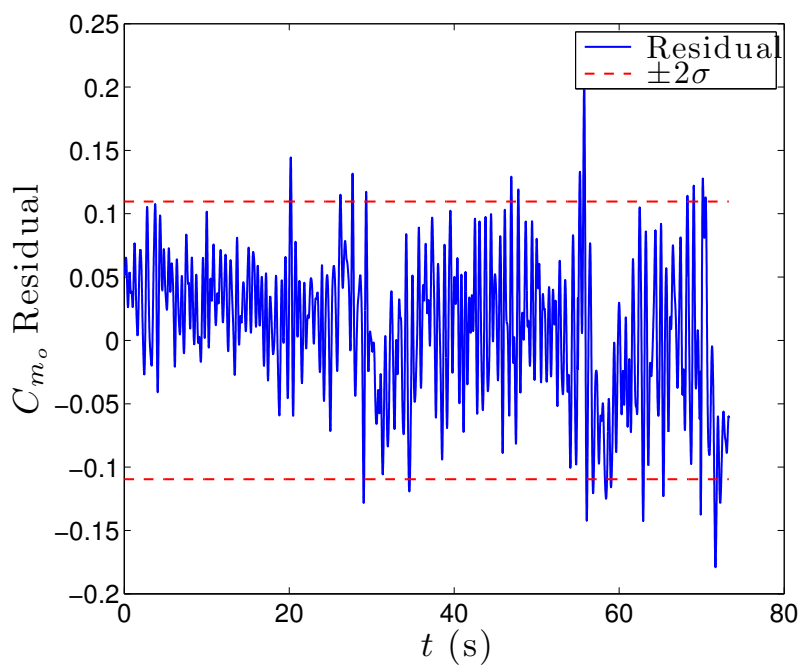

(k) Pitch moment coefficient residual.

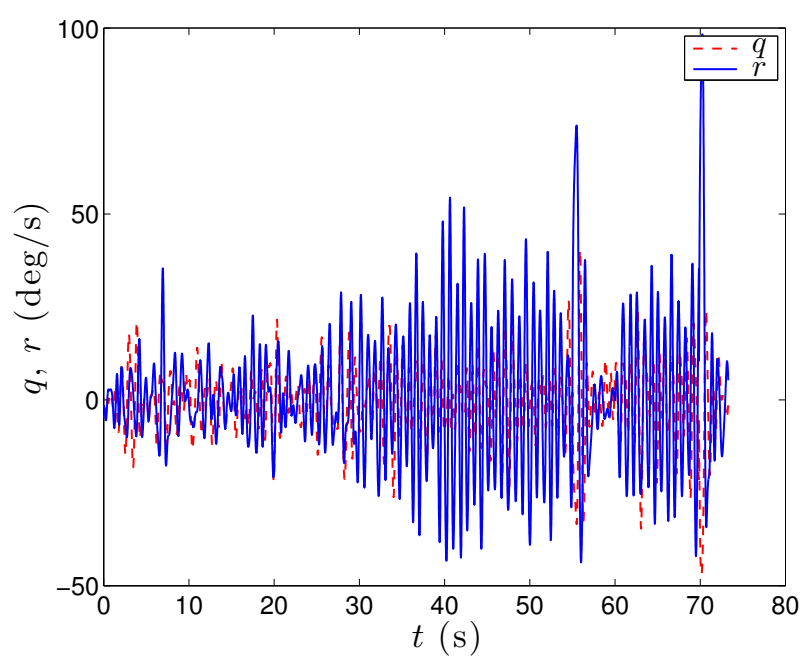

(h) $q$ and $r$ rotation rates.

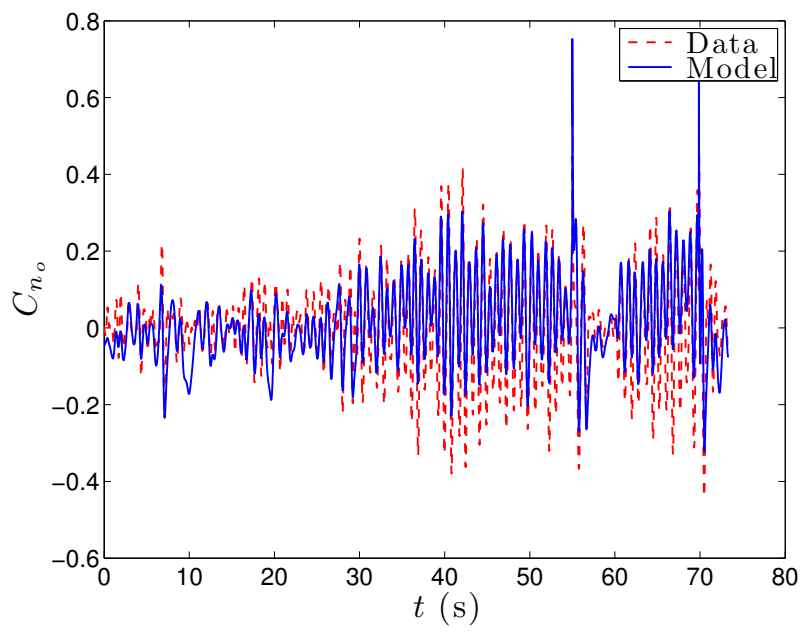

(j) Yaw moment coefficient.

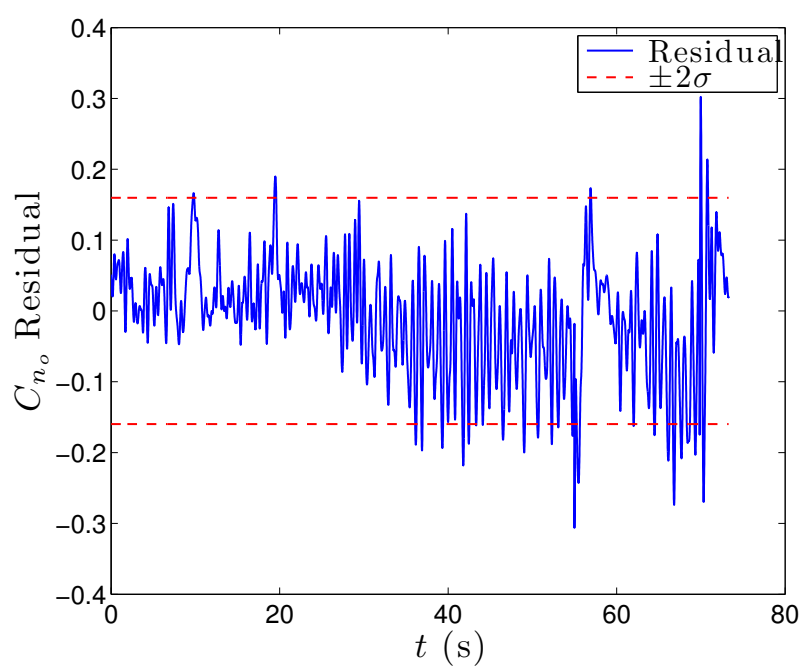

(1) Yaw moment coefficient residual.

Figure 5.3: Combined results. 


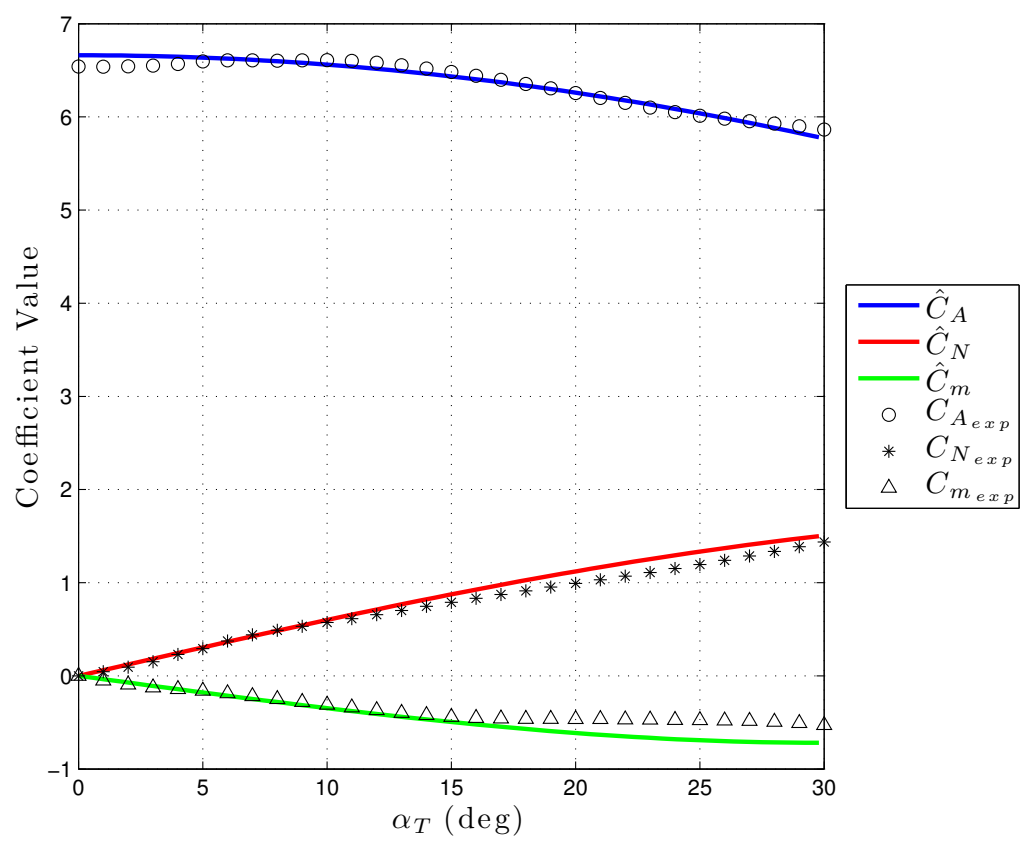

Figure 5.4: Static wind tunnel data for the selected lander configuration as compared to the results of the PID analysis.

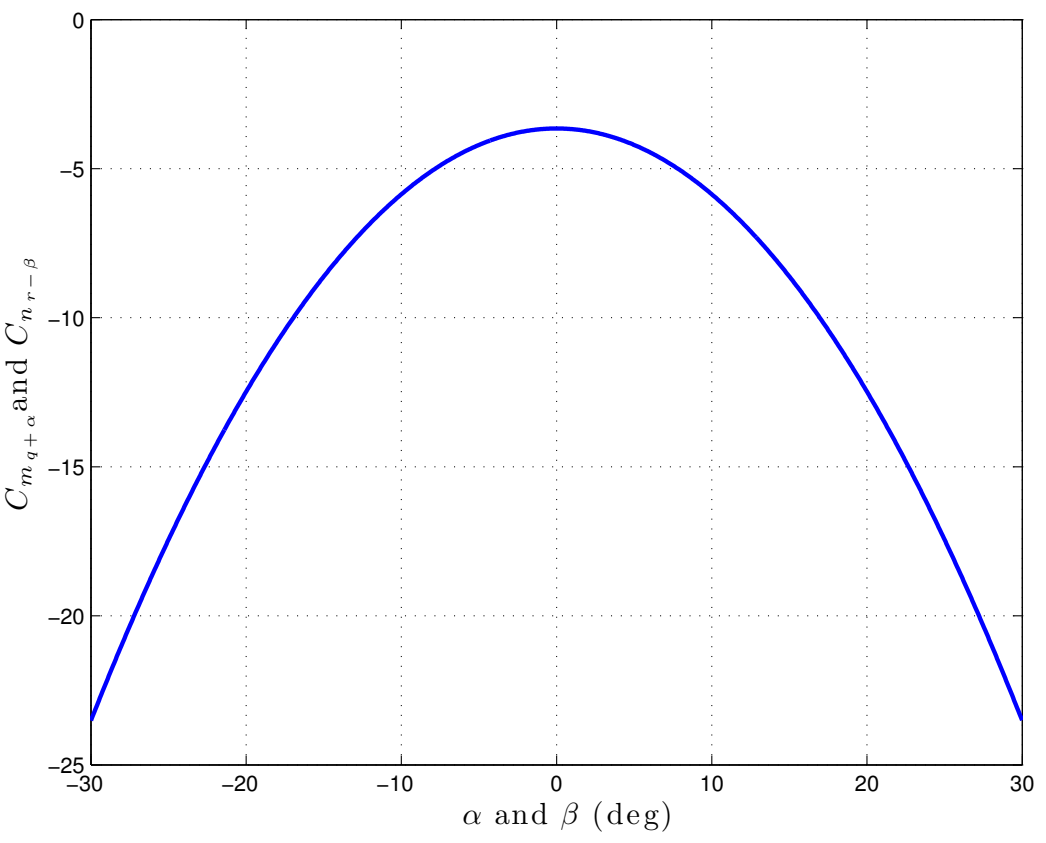

Figure 5.5: Estimated pitch and yaw damping function estimated by the PID analysis. 


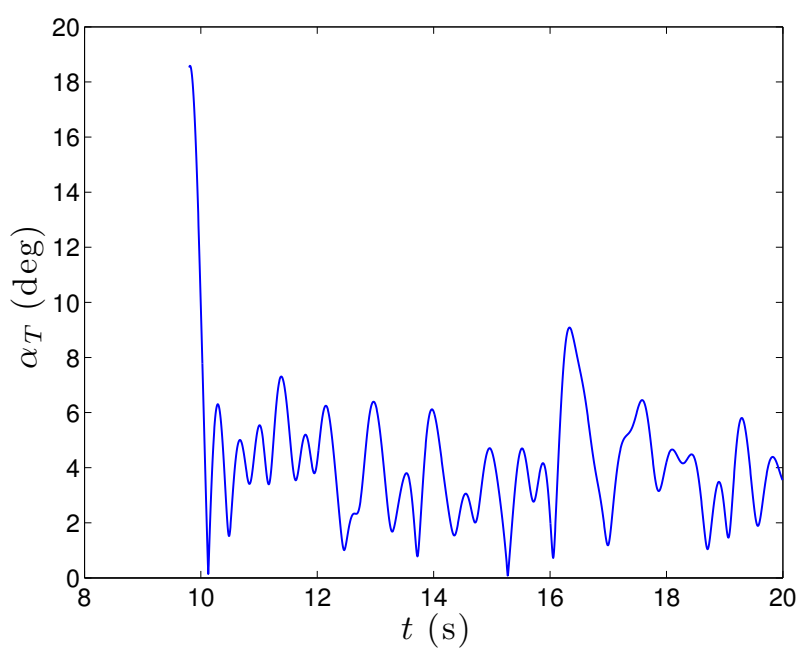

(a) Total angle of attack.

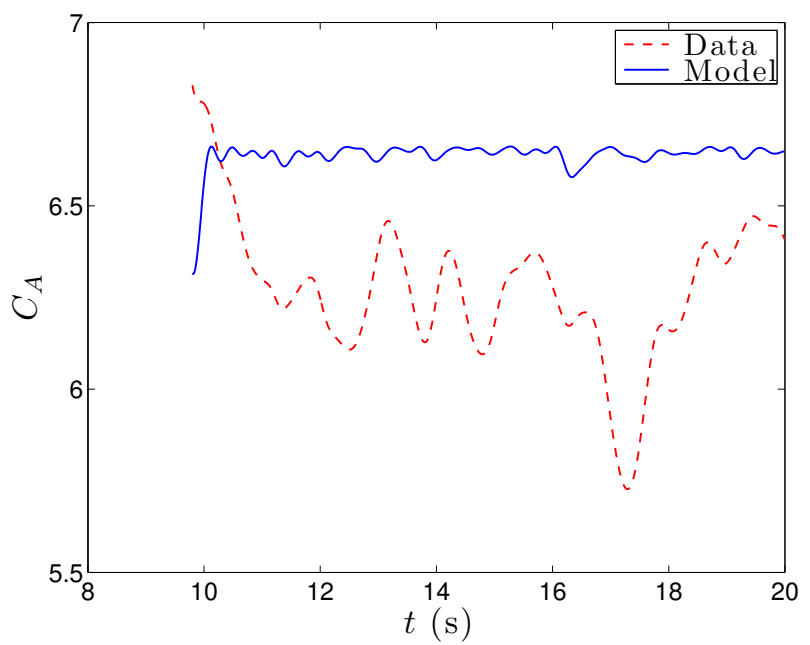

(c) Axial force coefficient.

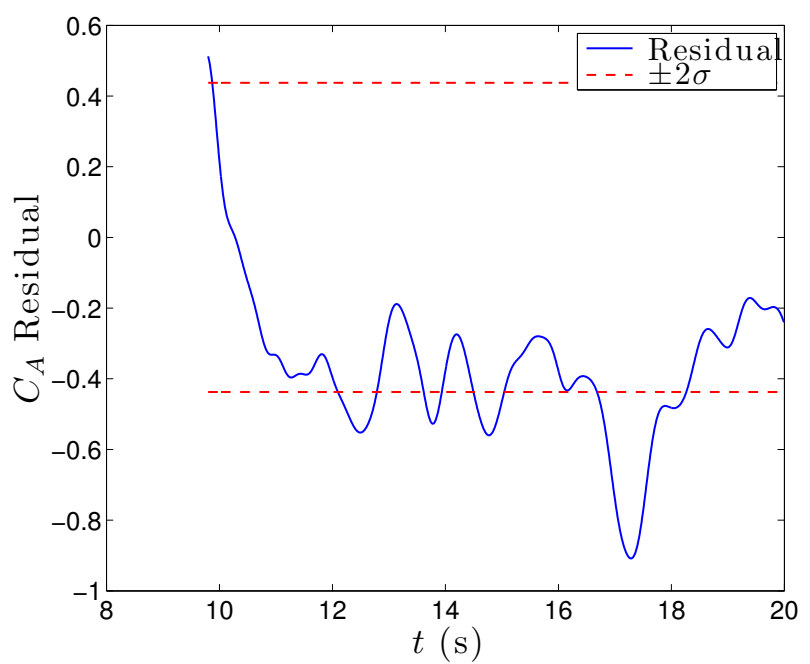

(e) Axial force coefficient residual.

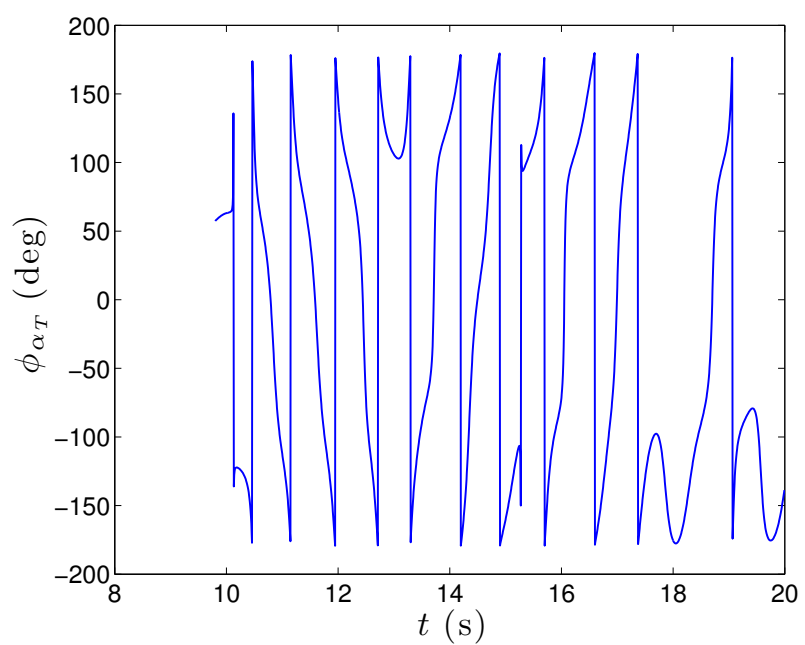

(b) Total angle of attack clock angle.

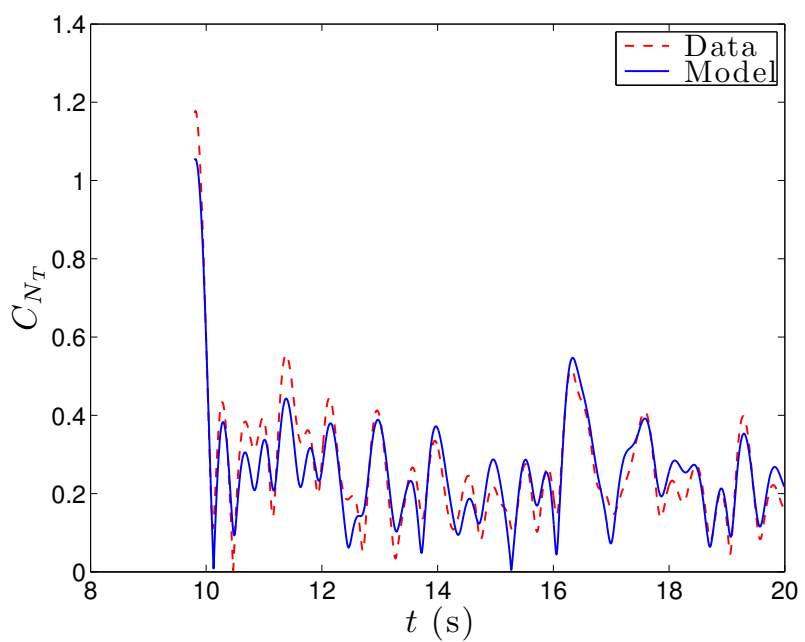

(d) Normal force coefficient.

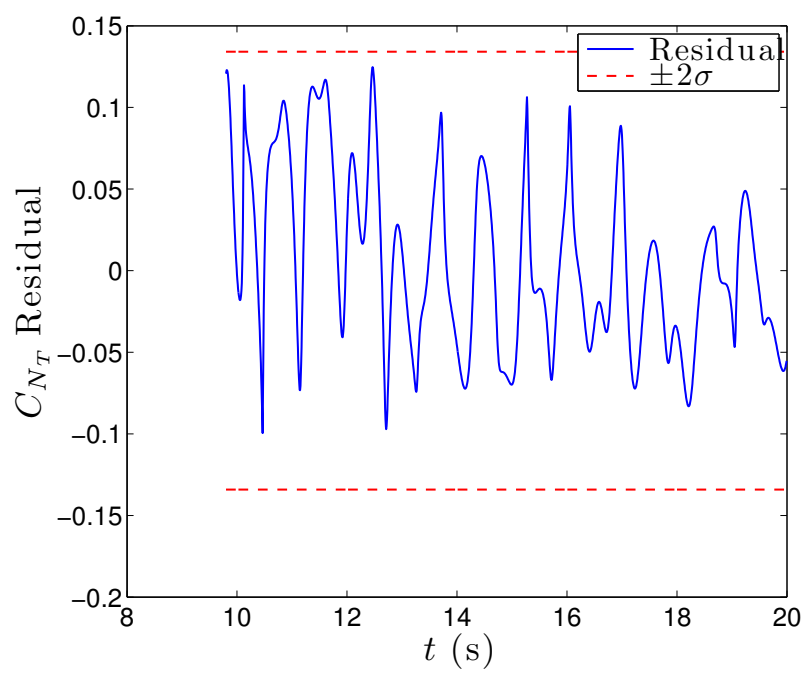

(f) Normal force coefficient residual.

Figure 5.6: Reconstruction of wind tunnel data not used in PID analysis. 


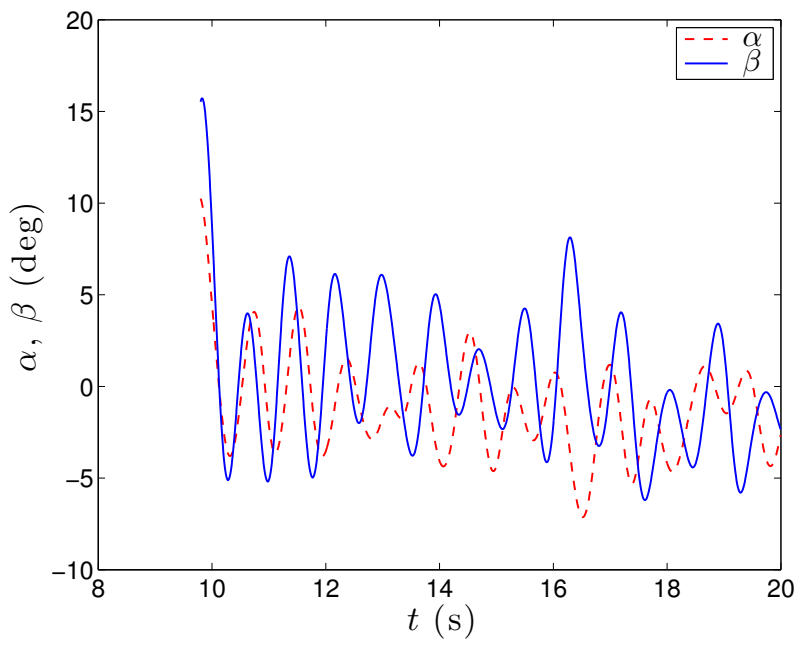

(g) $\alpha$ and $\beta$ angles.

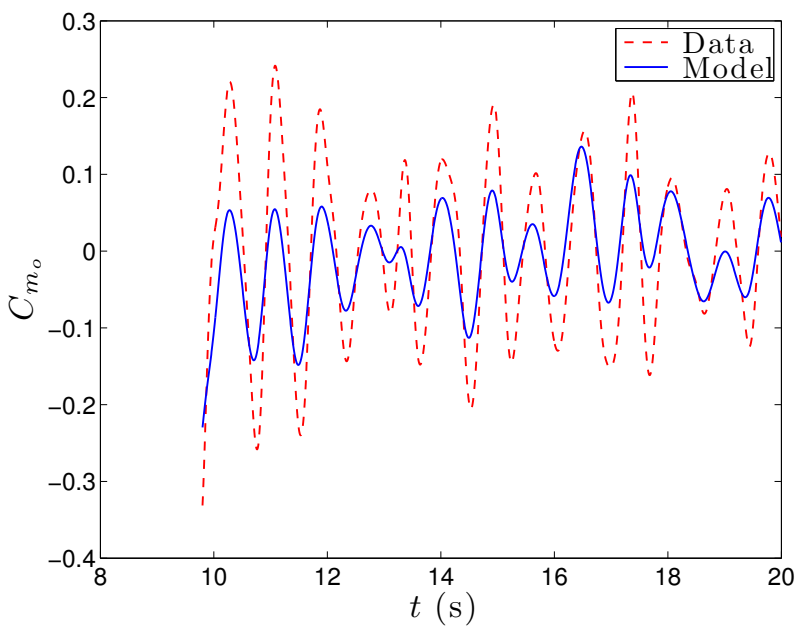

(i) Pitch moment coefficient.

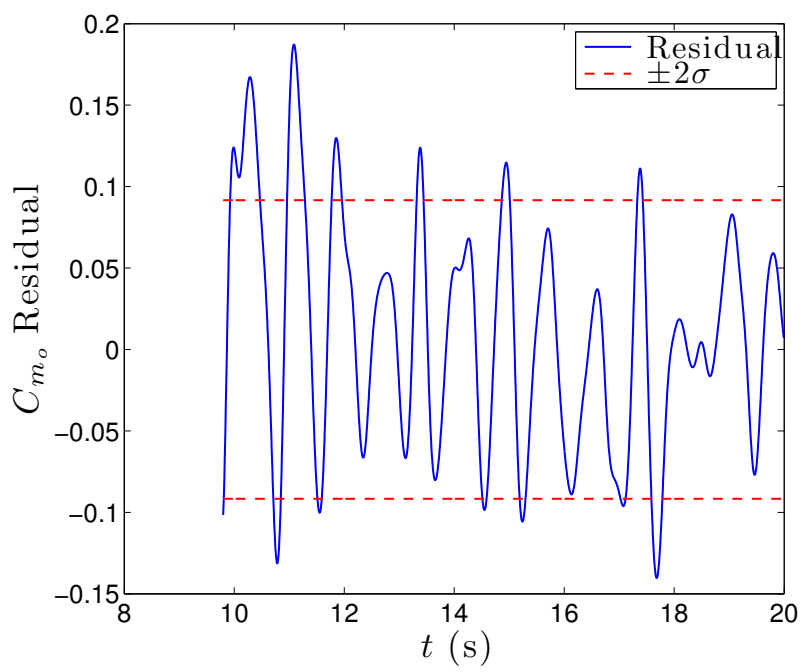

(k) Pitch moment coefficient residual.

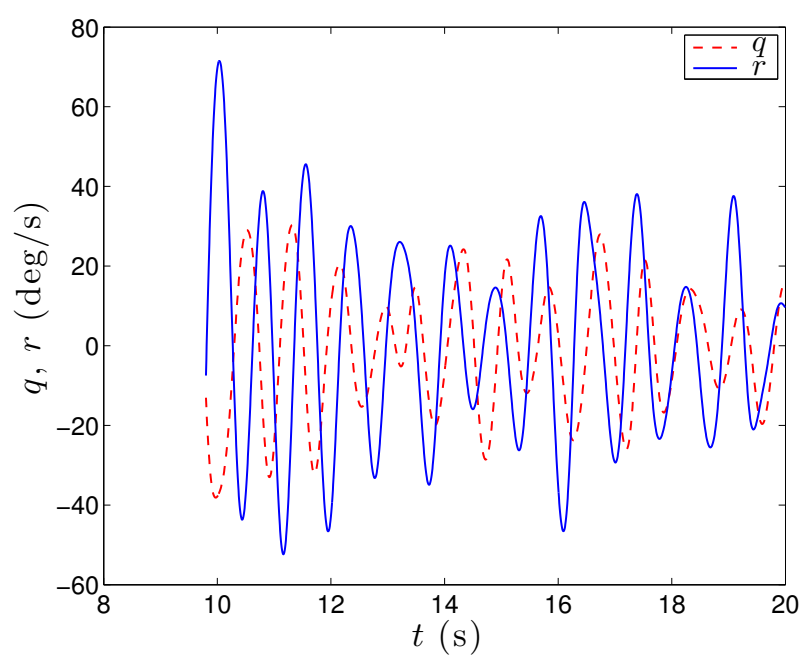

(h) $q$ and $r$ rotation rates.

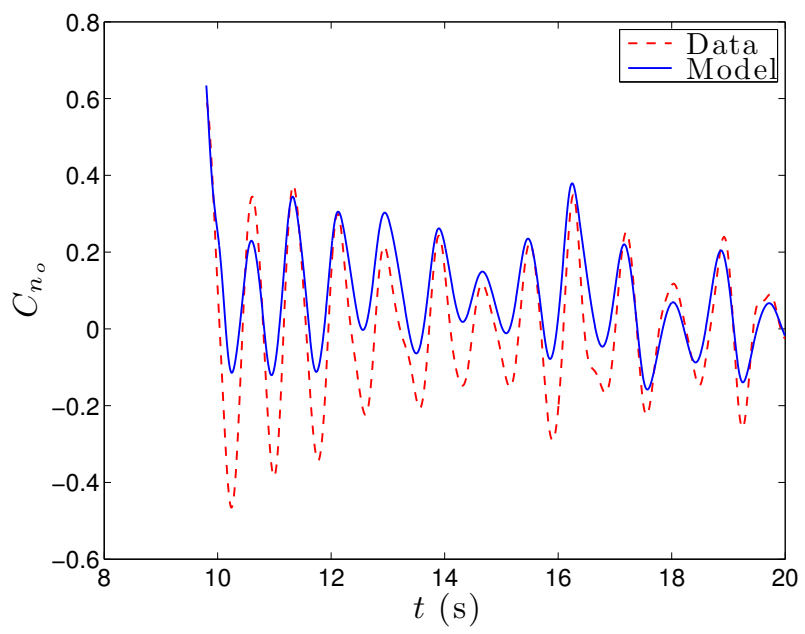

(j) Yaw moment coefficient.

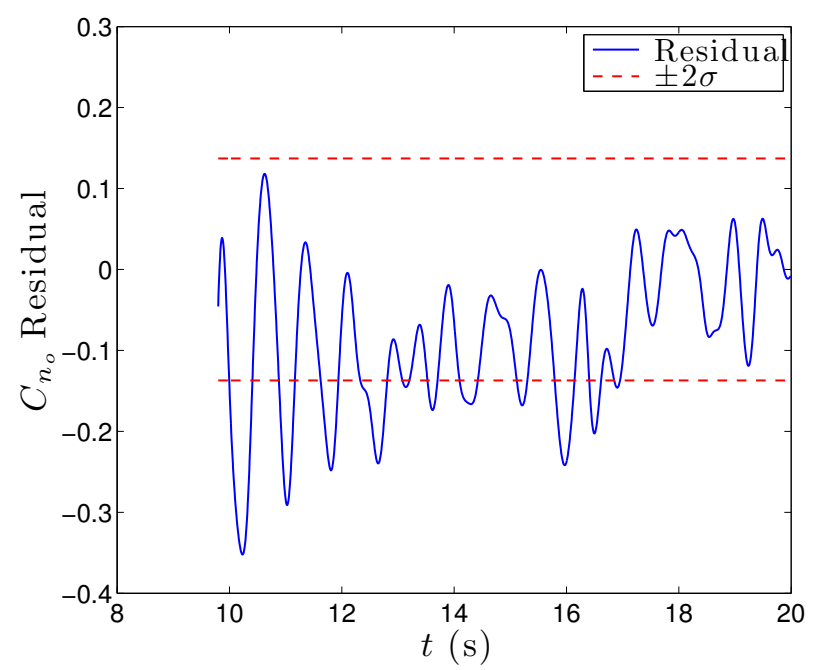

(l) Yaw moment coefficient residual.

Figure 5.6: Reconstruction of wind tunnel data not used in PID analysis. 


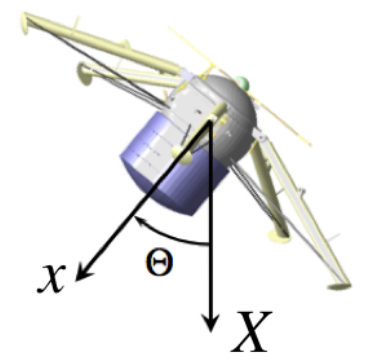

Figure 5.7: Definition of the off-vertical angle, $\Theta$. Adapted from image provided by NASA LaRC.

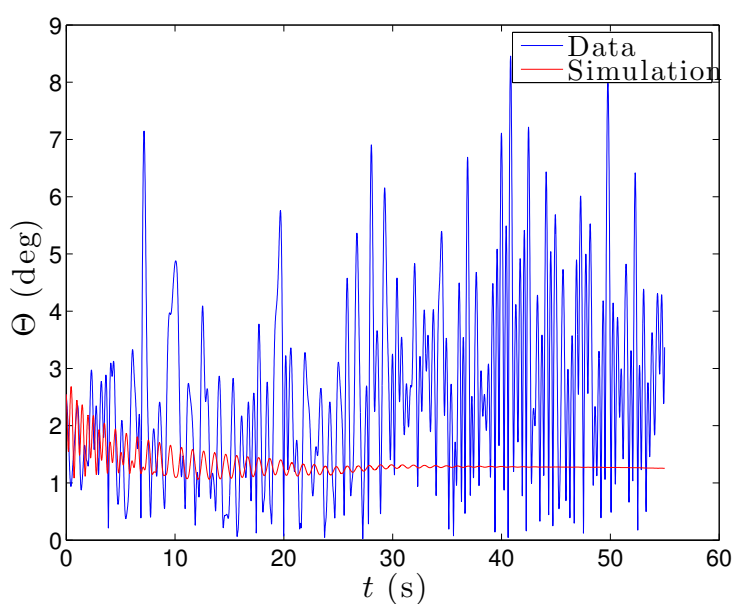

(a) $\Theta$ comparison.

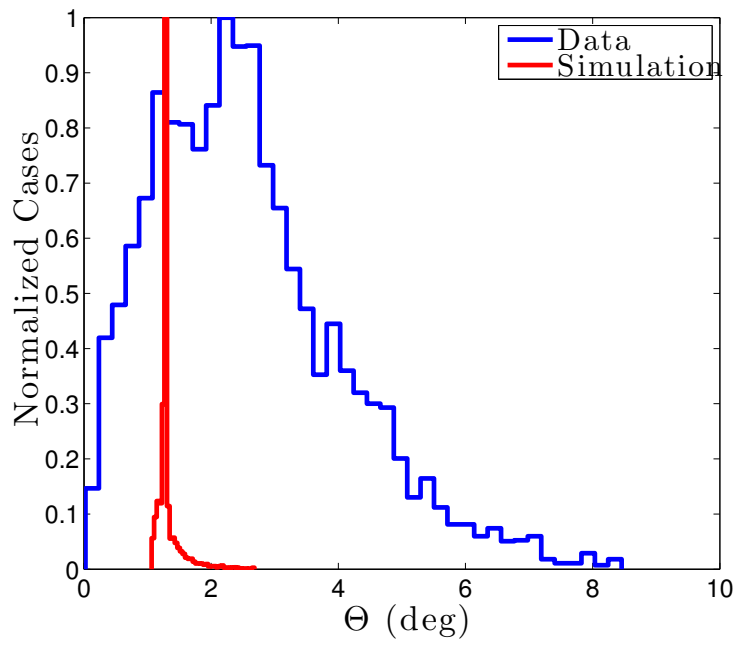

(c) $\Theta$ histogram.

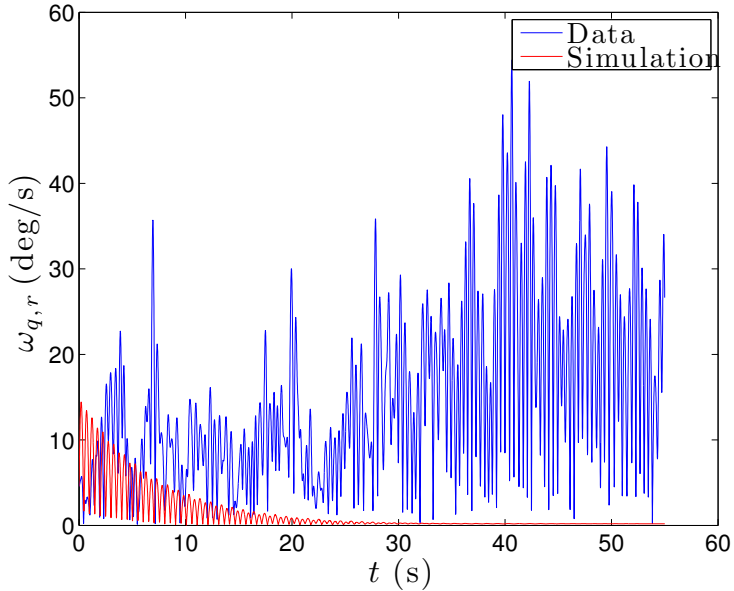

(b) $\omega_{q, r}$ comparison.

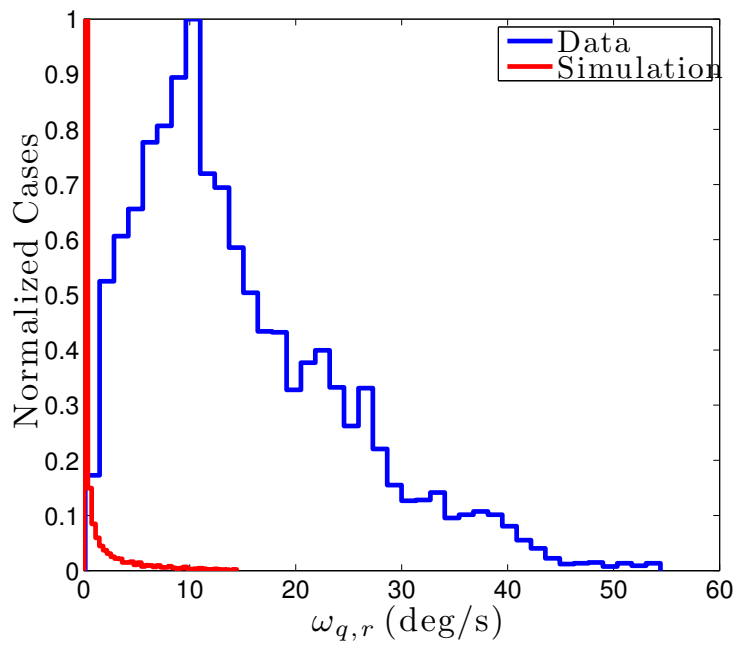

(d) $\omega_{q, r}$ histogram.

Figure 5.8: Comparison of test data against simulation results. 


\section{Chapter 6: Conclusions and Future Work}

\subsection{Conclusions}

A parameter identification analysis was conducted to determine the parameters present in an aerodynamic model of the SAGE lander. The aerodynamic coefficients were determined from experimental wind tunnel data collected in the NASA 20-ft VST. An ordinary least squares estimator was used to identify the parameters and their accuracies. The aerodynamic parameters identified by the current PID analysis represent the best estimates for the selected aerodynamic model. Conclusions can be drawn from the results that give insight into the dynamics of the SAGE lander and the behavior and accuracy of the aerodynamic model. The following conclusions about the SAGE Venus lander can be reached based on the current aerodynamic model and PID analysis of the dynamic wind tunnel data:

- The SAGE lander is statically and dynamically stable in the pitch and yaw planes. The static aerodynamic coefficients determined using the PID code were in agreement with those obtained from the static wind tunnel test. This gives credibility to the estimated dynamic aerodynamic parameters. The functional form of the static aerodynamic terms was known a priori due to previous testing but if the functional form is unknown static testing is preferred.

- There is a low total angle of attack limit cycle oscillation with random peak amplitudes that is not modeled by the aerodynamic model. This limit cycle oscillation is stochastic in nature and is likely caused by unsteady airflow around the lander. It is not known whether this limit cycle oscillation is due to the flow separating stochastically from the lander, wind tunnel turbulence, or a combination of both.

- The statistics of the experimental data as compared to simulated data shows that the off-vertical angle, $\Theta$, has an accurate mean steady state value for the simulation. However, the pitch and yaw rotation rate, $\omega_{q, r}$, is damped out and has a steady state value of zero. This difference can be attributed to the stochastic limit cycle oscillation discussed in the above bullet.

- Despite the fact that the static aerodynamics of the lander are estimated quite accurately by the PID code, static wind tunnel tests should still be performed for similar 
analyses. The existing static wind tunnel data was used in the aerodynamic model determination and helped to validate the results of the PID analysis.

Many issues with the current analysis can be attributed to behaviors of the PID code. The accuracy of the code factors into the conclusions made about the results of the SAGE lander. The following conclusions can be made about the PID code and its impact on the results:

- The code has difficulty identifying the aerodynamics at low total angles of attack, and low pitch and yaw rotation rates. This is true even for simulated data where the answer is known and the aerodynamic coefficients are determined with the same aerodynamic model that the PID code uses. The squared and cubic terms are overestimated and exaggerated to capture small curvatures in the functions for the coefficients given the small range of wind angles present in the test data. The larger range of wind angles in the perturbed data lend themselves much better to parameter identification.

- The tether model is an approximation and may have errors. The current tether model assumes a force that only varies with the free stream velocity in the tunnel but does not account for the motion of the model in the tunnel. For example if the model is moving vertically downwards in the tunnel the tether carries momentum that tends to oppose this motion. Such small effects are neglected in the current model but may have a larger impact that initially thought.

\subsection{Recommendations for Future Work}

The current PID analysis could be improved upon in several areas to increase the overall accuracy and obtain better results. The following represent the areas that have the largest potential for improvement:

- A more in-depth aerodynamic model optimization analysis should be conducted to possibly identify a better aerodynamic model for the lander. This would give a best model that can be proven mathematically as such. Although such an analysis was conducted to a limited extent, the results were inconclusive. A more thorough model identification analysis investigation may yield better results. In the current analysis parameters that relied on terms such as airspeed displayed strong correlations but were neglected because they did not physically make sense. The more in-depth system identification analysis may remedy these issues.

- Other PID methods should be considered. The ordinary least squares used in the analysis works well but there may be better methods. The only other parameter identification method that was considered in the current analysis was a simplex optimization method which worked but was not as repeatable. Ordinary least squares is an equation error method of which its results have strong predictive capabilities (e.g. 
situations where the current values of the regressors are known at each instant, such as the reconstructions) but have difficulty in simulations where it is used to estimate the values the next time step. Methods such as output error minimize the error between the state variables instead of the aerodynamic coefficient equation. Such methods may yield results that are better suited to simulations.

- The tether force model could be improved. The current tether model is a function of only the dynamic pressure. The model is presented in Appendix B. Other effects that could affect the tether force, for example the model's position in the test section, are not considered in the present tether force model. Improving the tether model will remove errors that will otherwise show up as errors in the aerodynamic coefficients.

- Improved testing techniques could yield data that lends itself better to PID analyses. This could include testing the SAGE lander in the VST without the use of the tether. A drop test of the lander should be conducted to remove the possibility that turbulence in the tunnel is interfering with the dynamics of the lander. 


\section{References}

[1] NASA/Jet Propulsion Laboratory/California Institute of Technology, "NASA Facts: Surface and Atmosphere Geochemical Explorer," 400-1404, April 21, 2010. (Downloaded on June 14, 2013, SAGE Fact Sheet-p6_for print_April-21-2010.pdf, lasp.colorado.edu/ sage/archives/PDF/)

[2] NASA/Langley Research Center, "Langley Research Center's 20-Foot Vertical Spin Tunnel," NP-2008-11-122-LaRC, November, 2008. (Downloaded on June 26, 2013, wWw.aeronautics.nasa.gov/atp/facilities/vst/index.html)

[3] Morelli, E. A., "Estimating Noise Characteristics from Flight Test Data Using Optimal Fourier Smoothing," Journal of Aircraft, Vol. 32, No. 4, July-August 1995, pp. 689-695.

[4] Klein, V. and Morelli, E.A. Aircraft System Identification: Theory and Practice, AIAA Education Series, AIAA, Reston, VA, 2006.

[5] Mitcheltree, R. A., Fremaux, C. M., Yates, L. A., "Subsonic Static and Dynamic Aerodynamics of Blunt Entry Vehicles," AIAA Paper 99-1020, 1999.

[6] Morelli, E. A., "Aerodynamic Parameter Estimation for the X-43A (Hyper X) from Flight Data," AIAA Paper 2005-5921, 2005. 


\section{Appendix A: Wind Tunnel Test Data}

The following are the static and dynamic wind tunnel data collected during testing of the SAGE lander. Only the data that applies to the current PID analyses are included. The static data were collected in NASA ARC's FML 48 in by 32 in subsonic wind tunnel. The dynamic wind tunnel data was collected in NASA LaRC's 20-ft Vertical Spin Tunnel. These dynamic data are further separated into unperturbed and perturbed data types.

\section{A.1 Static Data}

All three force and moment components were obtained from the internal wind tunnel balance for multiple values of $\alpha_{T}$ and $\phi_{\alpha_{T}}$. The force and moment components reduce to the aerodynamic coefficients: $C_{X}, C_{Y}, C_{Z}, C_{l_{o}}, C_{m_{o}}, C_{n_{o}}$. By imposing the assumption of axisymmetry, an axisymmetric model was devised in which the value varies with $\alpha_{T}$ but remained constant

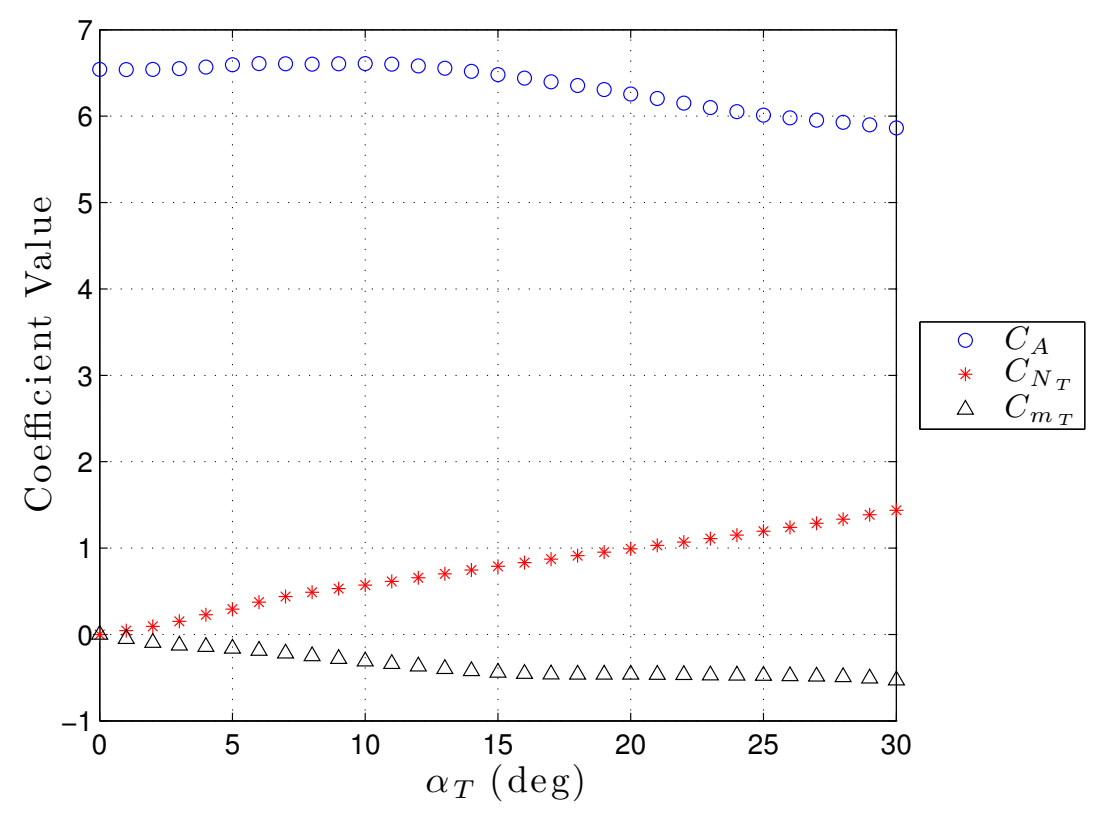

Figure A.1: Static wind tunnel test data. 
with $\phi_{\alpha_{T}}$.

\section{A.2 Dynamic Data}

\section{A.2.1 Unperturbed Data}

These are the raw data used in the analysis of the unperturbed data set. It is the first 55 seconds of Test Block 2 Run 2 of the dynamic wind tunnel test.

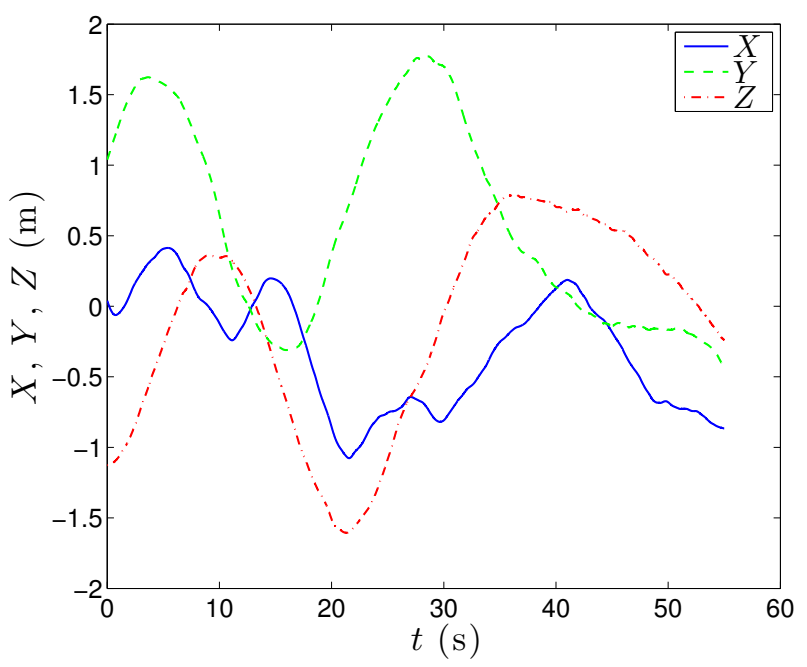

(a) $X, Y$, and $Z$ raw data.

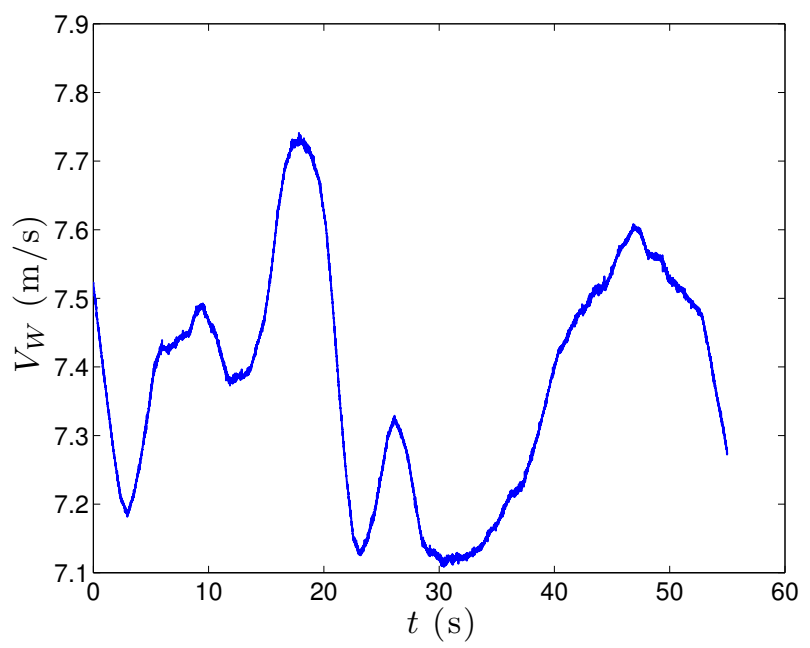

(c) $V_{W}$ raw data.

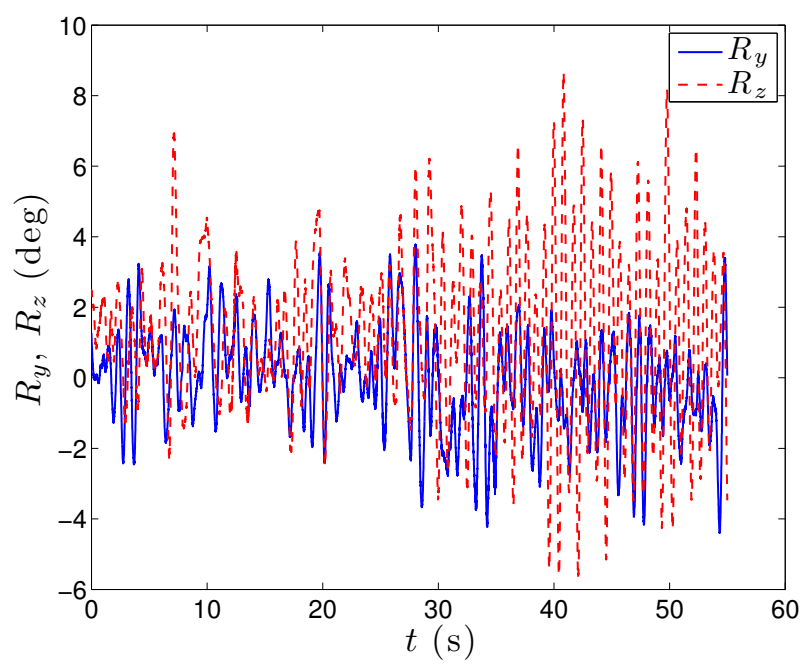

(b) $R_{y}$ and $R_{z}$ raw data.

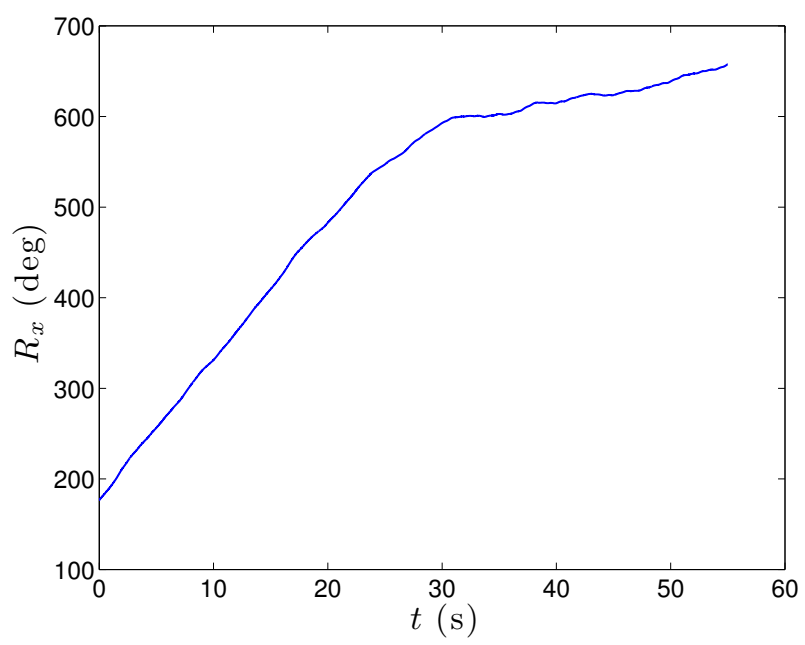

(d) $R_{x}$ raw data.

Figure A.2: Raw unperturbed wind tunnel data. 


\section{A.2.2 Perturbed Data}

\section{Perturbation 1}

These are the raw data used in the first perturbation of the perturbed data set. It consists of the first perturbation of Test Block 3 Run 2 of the dynamic wind tunnel test during the time window from 5.14 to 20 seconds.

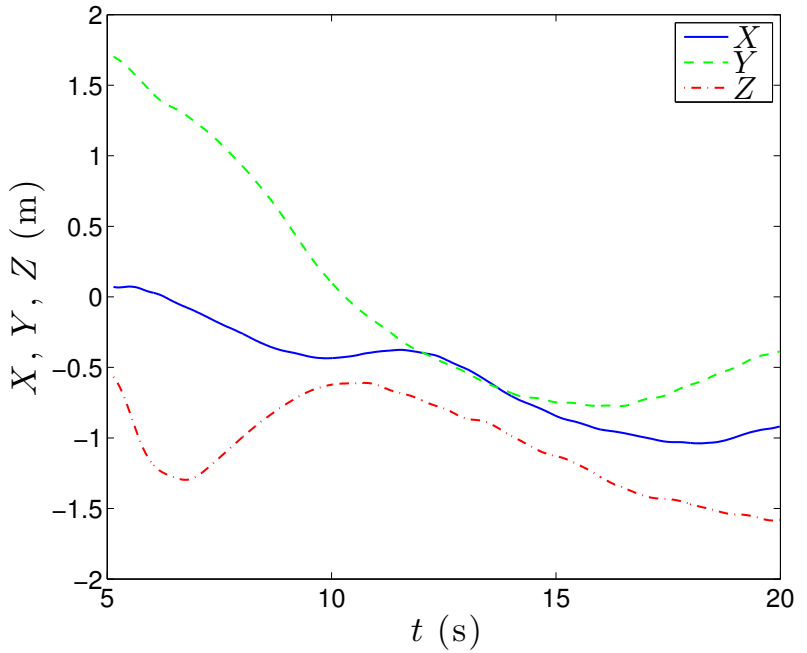

(a) $X, Y$, and $Z$ raw data.

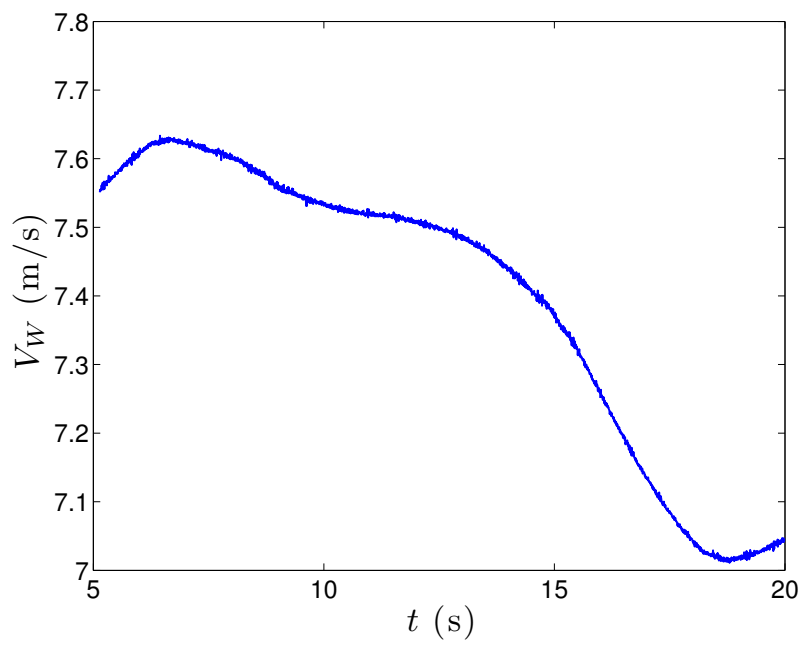

(c) $V_{W}$ raw data.

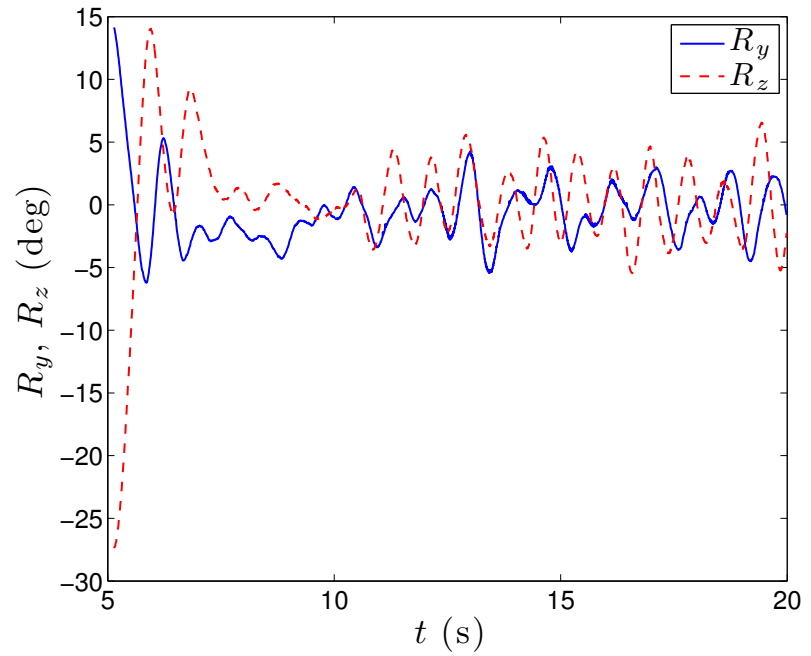

(b) $R_{y}$ and $R_{z}$ raw data.

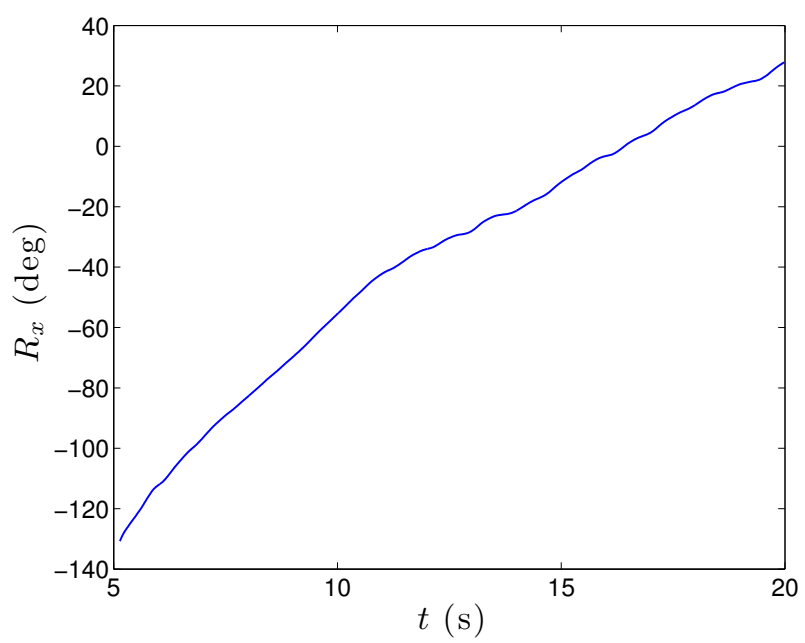

(d) $R_{x}$ raw data.

Figure A.3: Perturbation 1 raw wind tunnel data. 


\section{Perturbation 2}

These are the raw data used in the second perturbation of the perturbed data set. It consists of the sixth perturbation of Test Block 3 Run 3 of the dynamic wind tunnel test during the time window from 186.56 to 190 seconds.

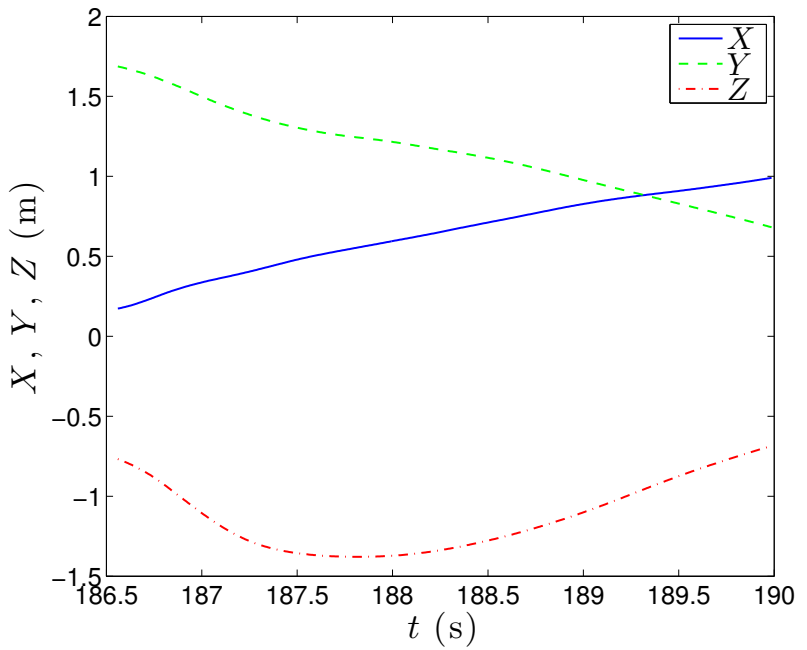

(a) $X, Y$, and $Z$ raw data.

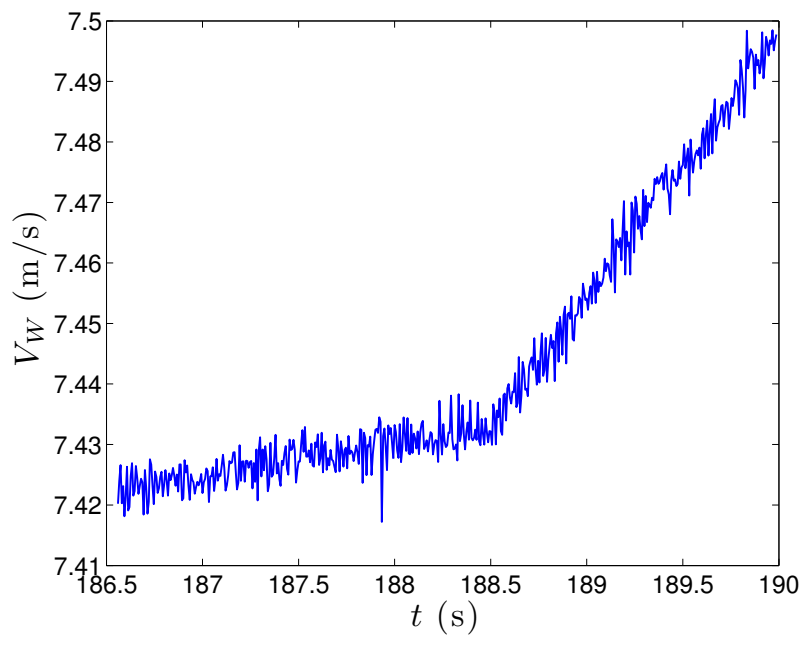

(c) $V_{W}$ raw data.

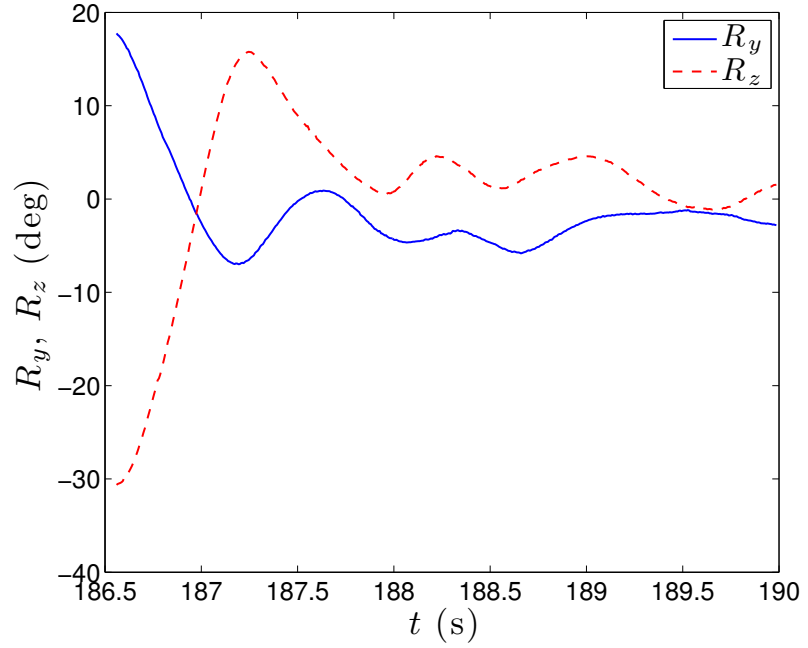

(b) $R_{y}$ and $R_{z}$ raw data.

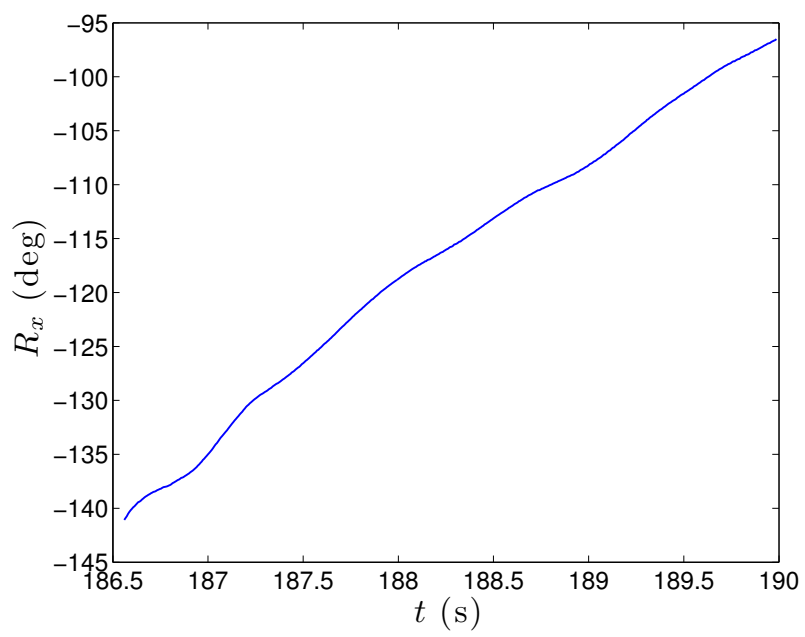

(d) $R_{x}$ raw data.

Figure A.4: Perturbation 2 raw wind tunnel data. Note the fuzzy appearance of $V_{W}$ due to noise content of the raw data. 


\section{Perturbation 3}

These are the raw data used for the predictive validation of the PID results in section 5.3.3. It consists of the first perturbation of Test Block 3 Run 3 of the dynamic wind tunnel test during the time window from 9.8 to 20.3 seconds.

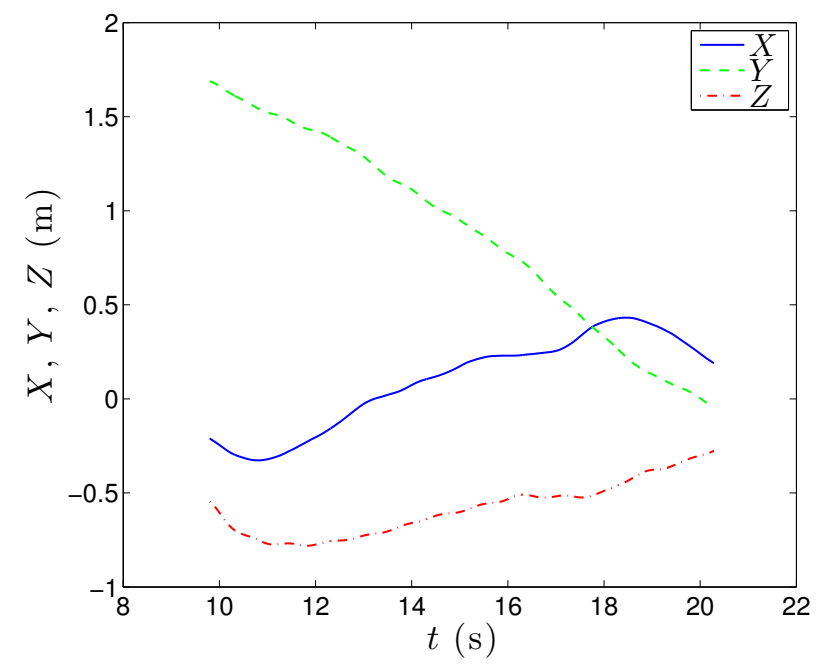

(a) $X, Y$, and $Z$ raw data.

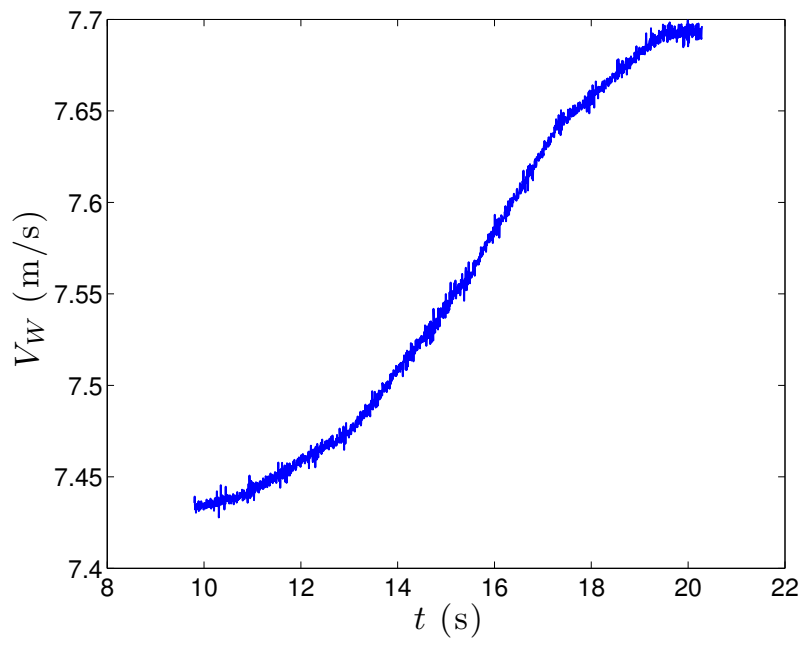

(c) $V_{W}$ raw data.

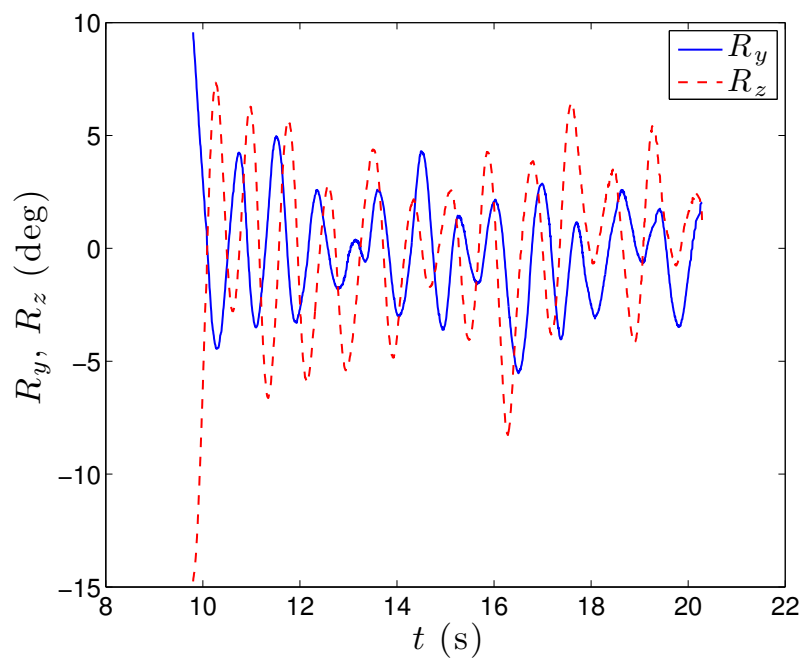

(b) $R_{y}$ and $R_{z}$ raw data.

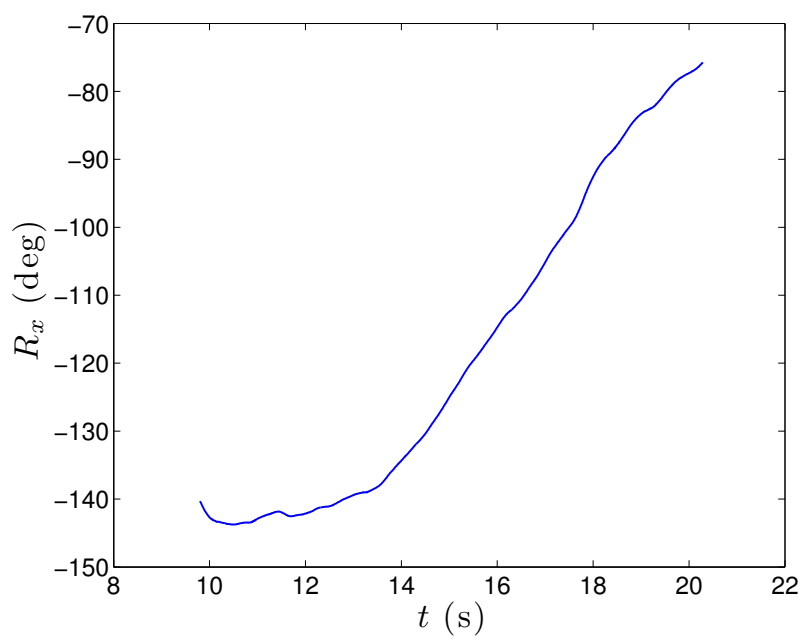

(d) $R_{x}$ raw data.

Figure A.5: Perturbation 3 raw wind tunnel data. 


\section{A.3 Noise Content of the Dynamic Data}

The following figures show the frequency spectrum typical of the raw data. Table 3.1 shows the cutoff frequencies used in the smoothing routine to construct the Wiener filter. The figures are of the data in the unperturbed data set and are typical of the perturbed data set as well. The signal noise presented here only includes precision error. The mean and standard error of the noise are used as accuracy estimates.

\section{Wind Velocity Data}

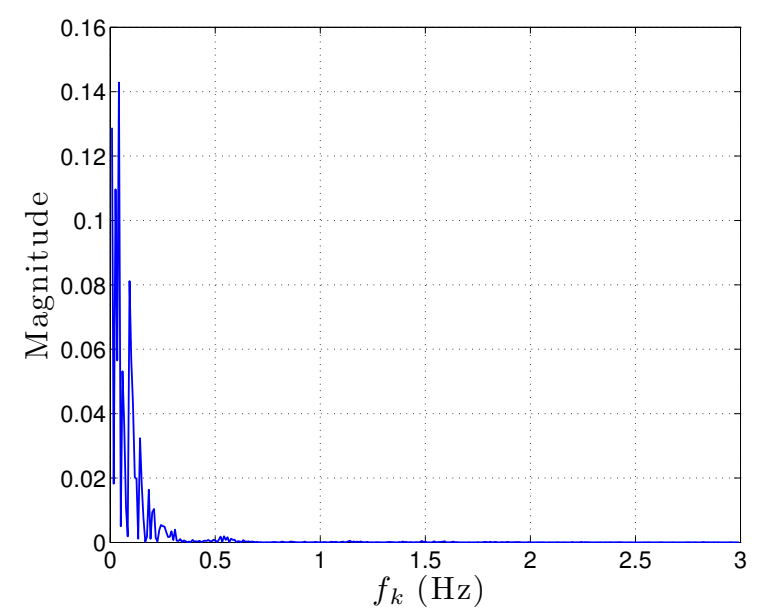

(a) Frequency spectrum for $V_{W}$.

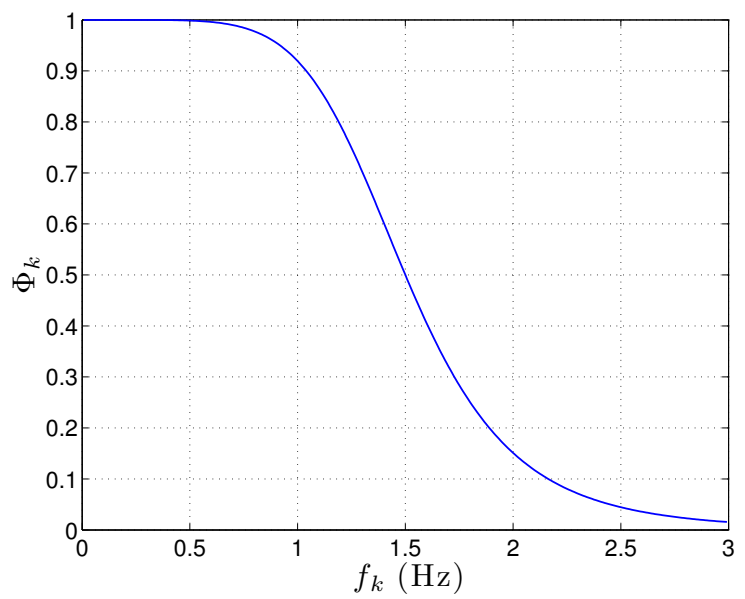

(c) Wiener filter designed for $V_{W}$.

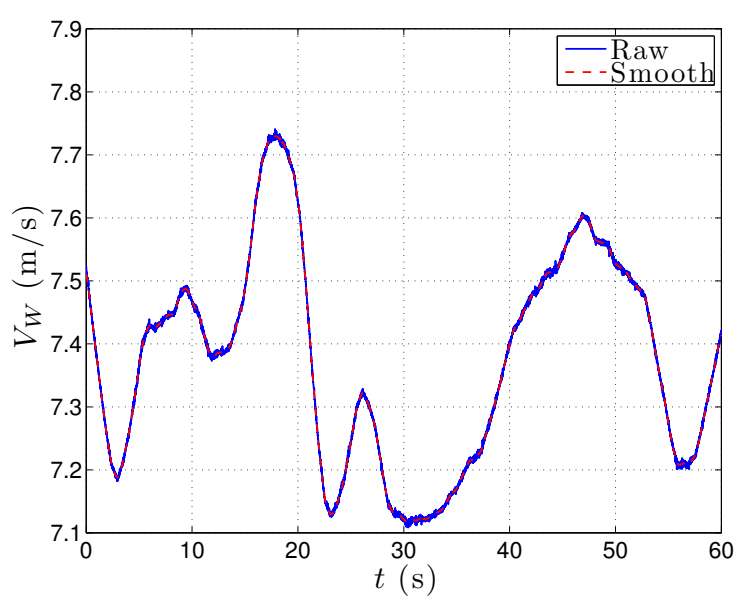

(b) $V_{W}$ signal comparison.

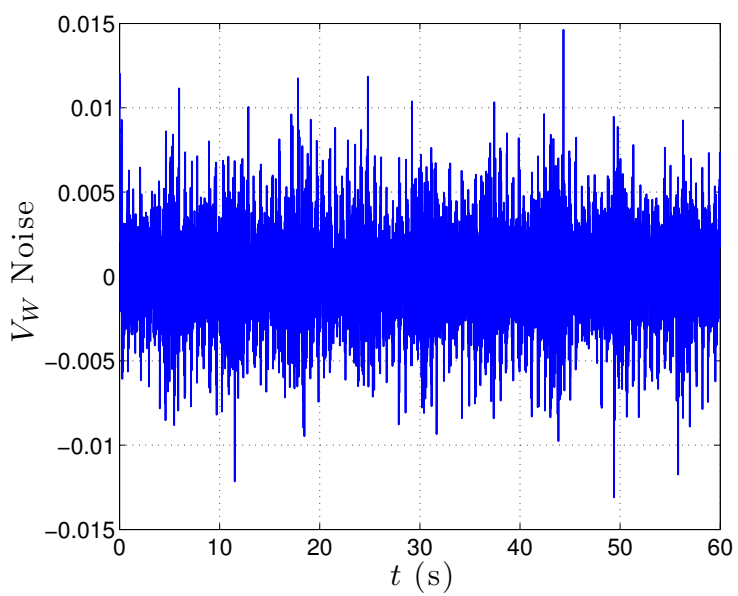

(d) $V_{W}$ signal noise.

Figure A.6: Typical noise content of $V_{W}$ data. The noise has a standard error of $s_{\nu}=$ $2.57 \times 10^{-3} \mathrm{~m} / \mathrm{s}$. 


\section{Position Data}

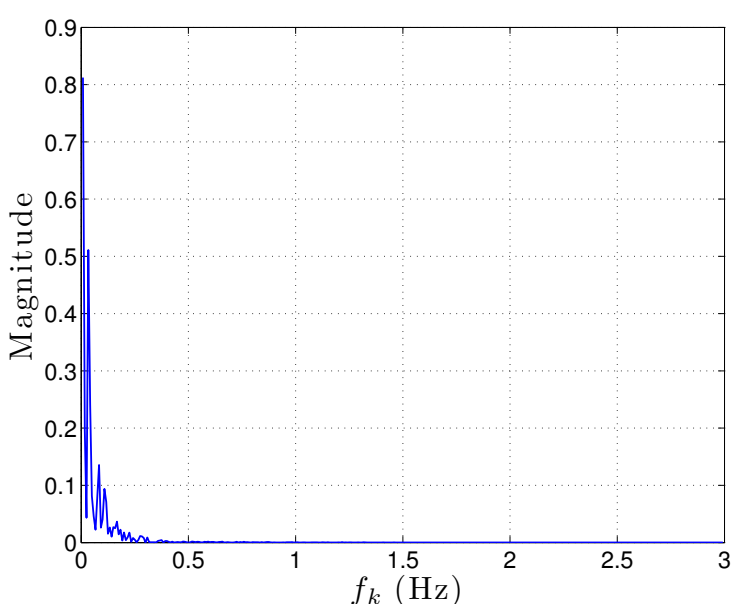

(a) Frequency spectrum for $X$.

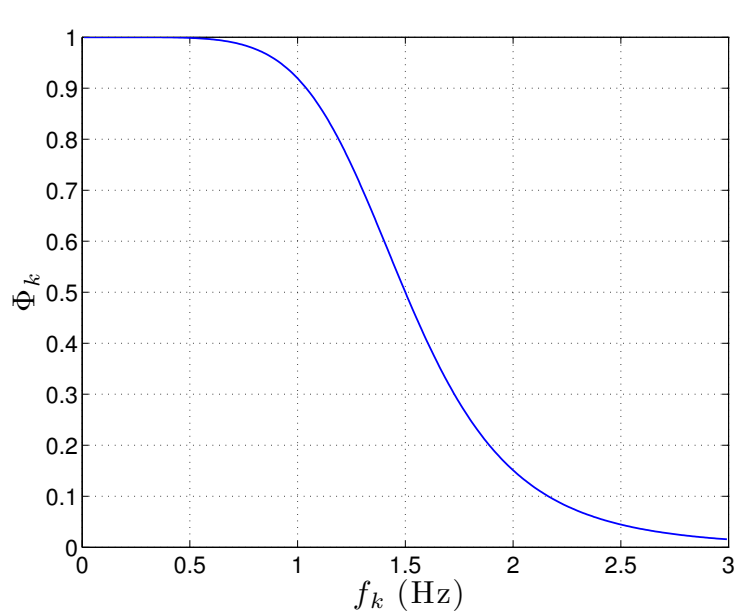

(c) Wiener filter designed for $X$.

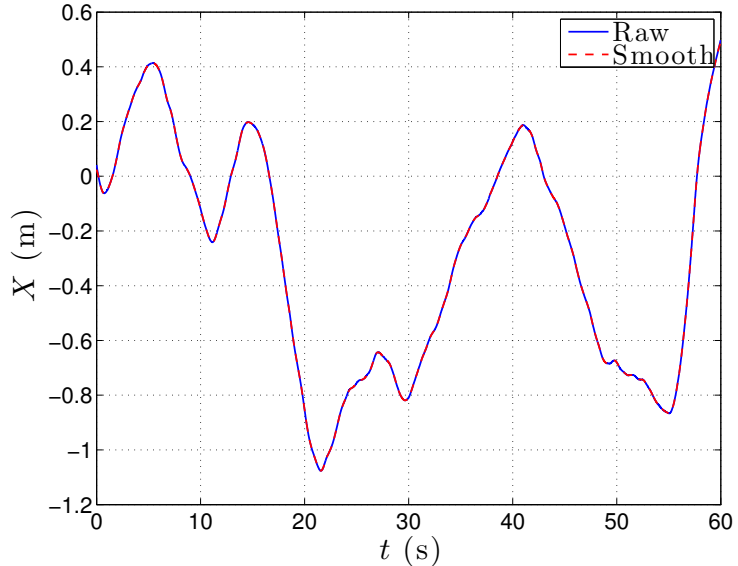

(b) $X$ signal comparison.

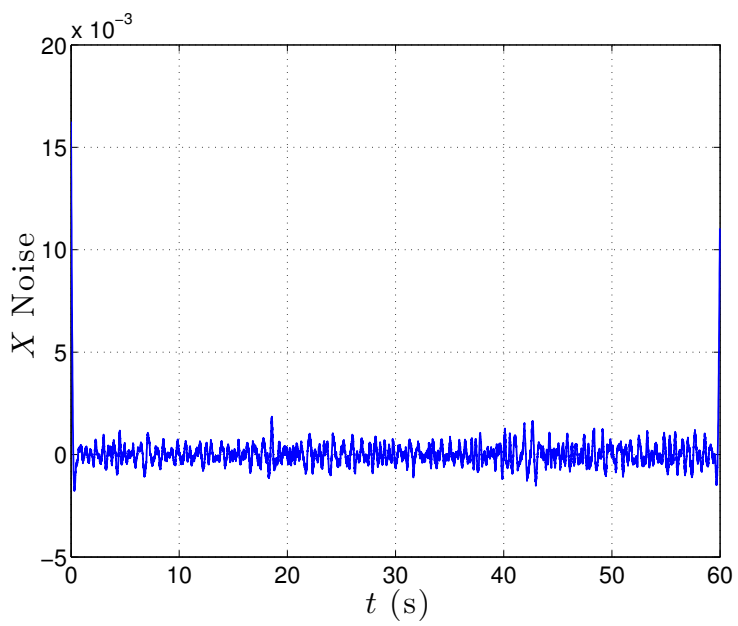

(d) $X$ signal noise.

Figure A.7: Typical noise content of $X$ position data. This is also representative of the noise content of the $Y$ and $Z$ data. The noise has a standard error of $s_{\nu}=7.50 \times 10^{-4} \mathrm{~m}$. 


\section{Pitch and Yaw Data}

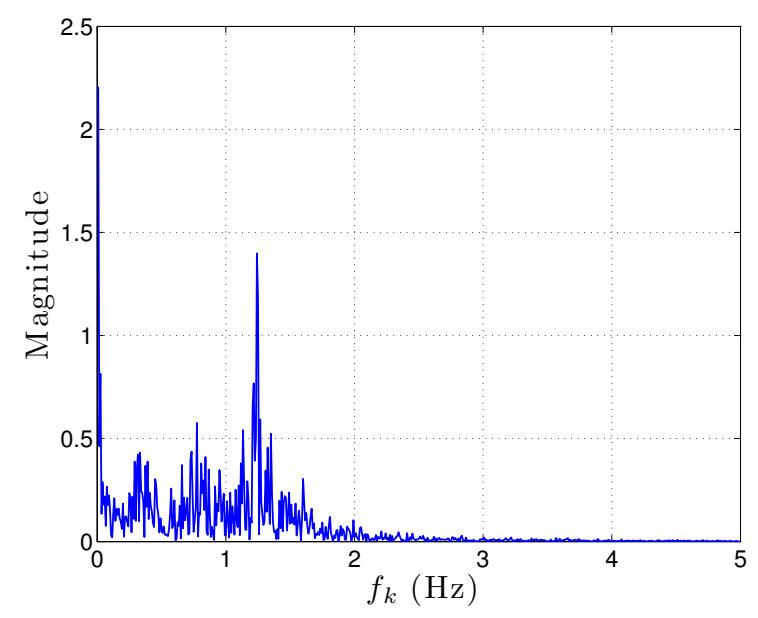

(a) Frequency spectrum for $R z$.

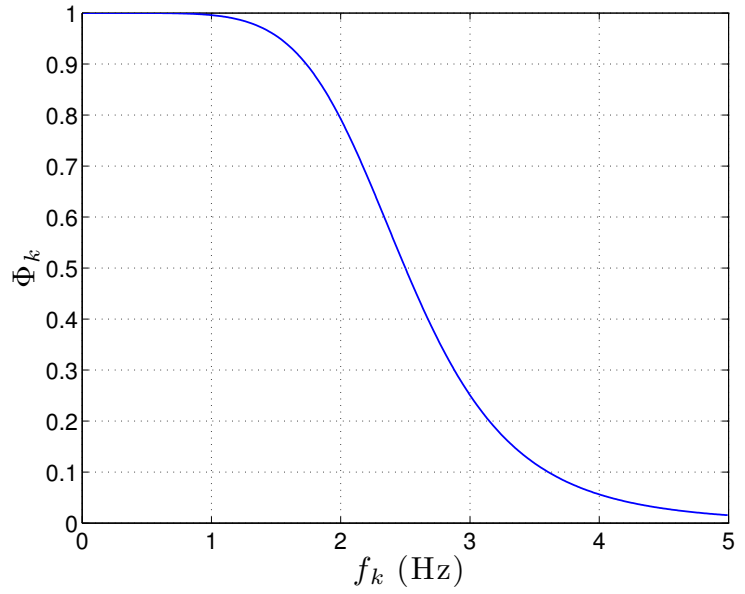

(c) Wiener filter designed for $R z$.

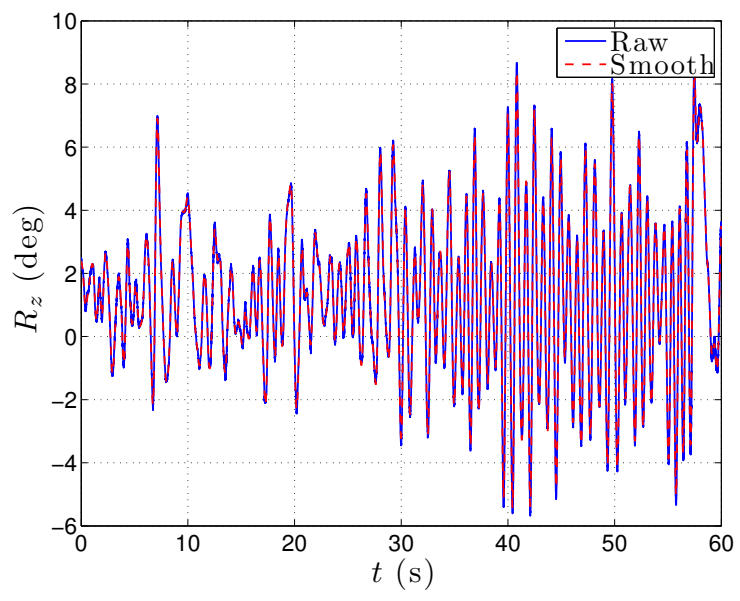

(b) $R z$ signal comparison.

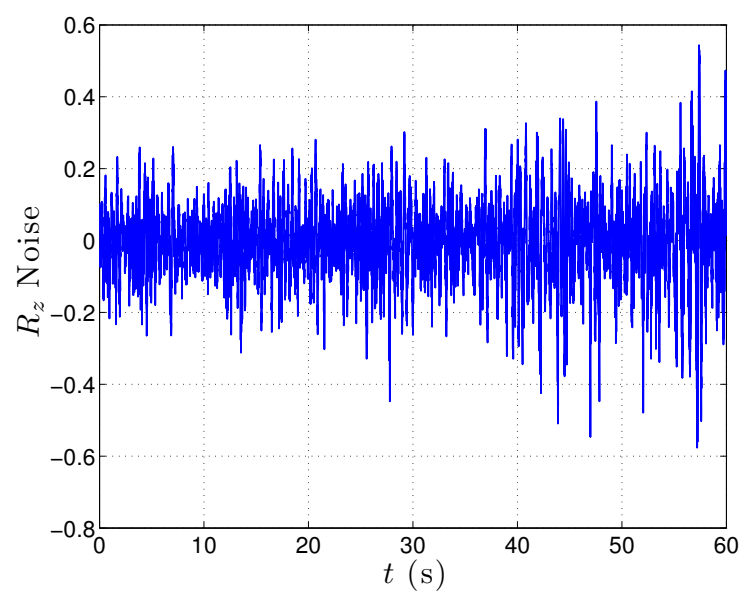

(d) $R z$ signal noise.

Figure A.8: Typical noise content of pitching angle, $R_{z}$, data. This is also representative of the noise content of the yaw angle, $R_{y}$, data. The noise has a standard error of $s_{\nu}=$ $1.10 \times 10^{-1}$ degrees. 


\section{Roll Data}

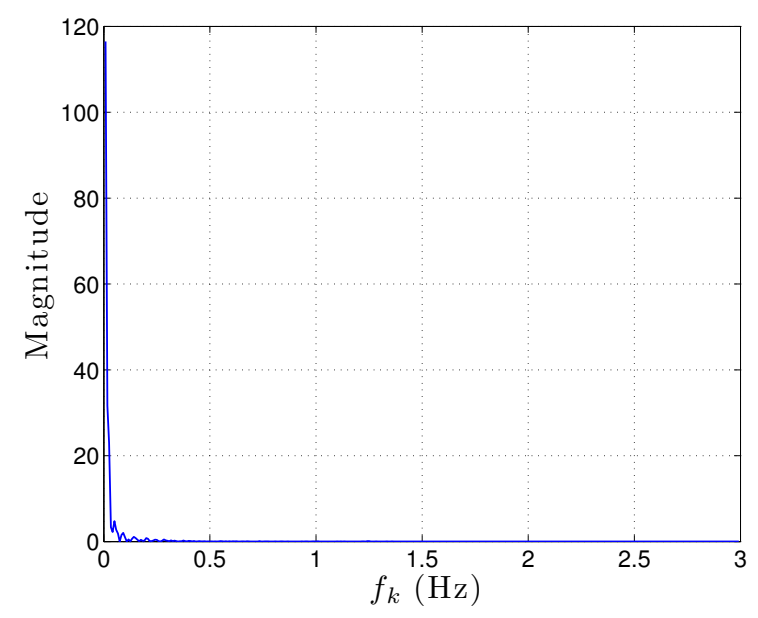

(a) Frequency spectrum for $R x$.

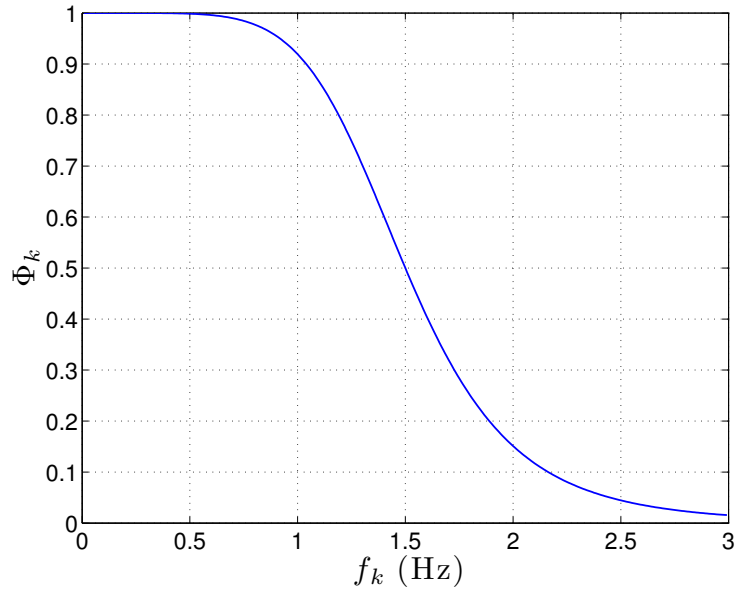

(c) Wiener filter designed for $R x$.

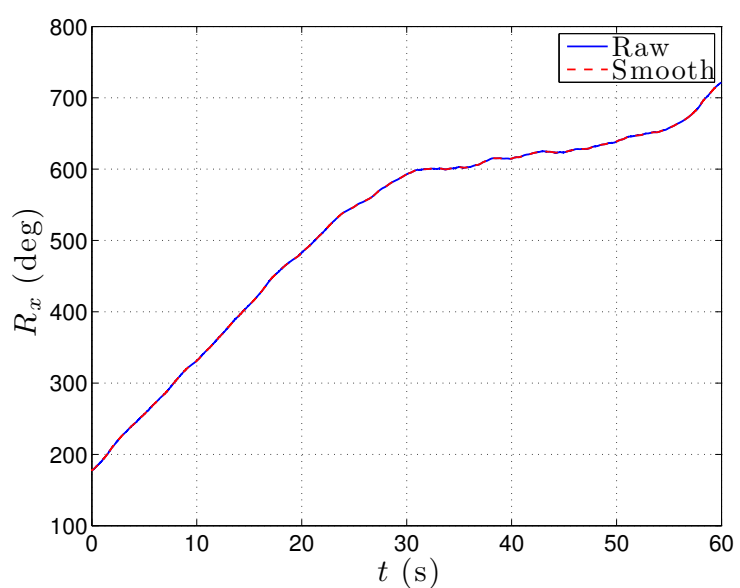

(b) $R x$ signal comparison.

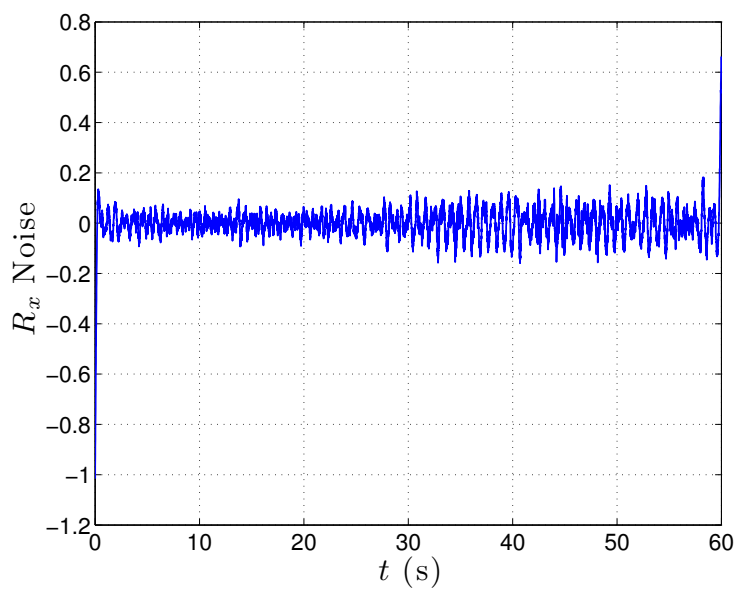

(d) $R x$ signal noise.

Figure A.9: Typical noise content of roll angle, $R_{x}$, data. The noise has a standard error of $s_{\nu}=6.23 \times 10^{-2}$ degrees. 


\section{Appendix B: Flight Mechanics Equations}

\section{B.1 Introduction}

The following are the flight mechanics equations used in the parameter identification analysis. The equations of motion use the state variables described in Section 3.4. This appendix presents the equations with minimum explanation or commentary. The symbols used are described in the front matter of this thesis. The equations were presented by Juan R. Cruz in the document "Equations of Motion for an Entry Vehicle." The equations were modified to suit the coordinate systems used in the VST data and to include the tether force.

\section{B.2 Assumptions}

The following assumptions were made in the derivation of the equations:

- Any effects of planetary rotation or curvature of the planetary surface are neglected.

- The planet fixed coordinate system is the same as defined in section 2.1.4 of this thesis.

- The origin of the body centered coordinate system is located at the center of mass and uses the state variables determined in section 3.3 of this thesis.

- The lander is axisymmetric in shape but not in mass distribution (i.e. the center of mass may be offset from the axis of symmetry).

\section{B.3 Rigid Body Equations of Motion}

The forces and moments on the lander are due to gravity, aerodynamics, and the tether. The vectorial sum of forces and moments are:

$$
\begin{gathered}
\mathbf{F}=\mathbf{F}_{g}+\mathbf{F}_{t r}+\mathbf{F}_{A} \\
\mathbf{M}=\mathbf{M}_{t r}+\mathbf{M}_{A}
\end{gathered}
$$

Note that there are no gravitational moments because the gravitational force acts at the center of mass. The inertial force and moment vectors are defined as: 


$$
\begin{gathered}
\mathbf{F}=F_{x} \mathbf{i}+F_{y} \mathbf{j}+F_{z} \mathbf{k} \\
\mathbf{M}=M_{x} \mathbf{i}+M_{y} \mathbf{j}+M_{z} \mathbf{k}
\end{gathered}
$$

The components of the vectors are calculated with the rigid body equations of motion, Eqs. (B.5) - (B.10).

$$
\begin{gathered}
F_{x}=m(\dot{u}+q w-r v) \\
F_{y}=m(\dot{v}+r u-p w) \\
F_{z}=m(\dot{w}+p v-q u) \\
M_{x}=I_{x x} \dot{p}-I_{x y} \dot{q}-I_{x z} \dot{r}-I_{y z} q^{2}+I_{y z} r^{2}-I_{x z} p q+I_{x y} p r+\left(I_{z z}-I_{y y}\right) q r \\
M_{y}=-I_{x y} \dot{p}+I_{y y} \dot{q}-I_{y z} \dot{r}+I_{x z} p^{2}-I_{x z} r^{2}+I_{y z} p q+\left(I_{x x}-I_{z z}\right) p r-I_{x y} q r \\
M_{z}=-I_{x z} \dot{p}-I_{y z} \dot{q}+I_{z z} \dot{r}-I_{x y} p^{2}+I_{x y} q^{2}+\left(I_{y y}-I_{x x}\right) p q-I_{y z} p r+I_{x z} q r
\end{gathered}
$$

\section{B.4 Gravitational Forces}

The gravity force vector is defined as:

$$
\mathbf{F}_{g}=F_{g, x} \mathbf{i}+F_{g, y} \mathbf{j}+F_{g, z} \mathbf{k}
$$

The components of the force vector are calculated with Eq. (B.2). The transformation matrix, Eq. (B.13), converts the gravity vector from the inertial coordinate system to the body fixed coordinate system.

$$
\left\{\begin{array}{l}
F_{g, x} \\
F_{g, y} \\
F_{g, z}
\end{array}\right\}=\left[T^{B E F}\right]^{T}\left\{\begin{array}{c}
m g \\
0 \\
0
\end{array}\right\}
$$

The transformation matrix is defined as:

$$
\left[T^{B E F}\right]=\left[\begin{array}{lll}
T_{11}^{B E F} & T_{12}^{B E F} & T_{13}^{B E F} \\
T_{21}^{B E F} & T_{22}^{B E F} & T_{23}^{B E F} \\
T_{31}^{B E F} & T_{32}^{B E F} & T_{33}^{B E F}
\end{array}\right]
$$

and the elements are:

$$
\begin{gathered}
T_{11}^{B E F}=\cos R_{z} \cos R_{y} \\
T_{12}^{B E F}=-\sin R_{z} \cos R_{y} \\
T_{13}^{B E F}=\sin R_{y}
\end{gathered}
$$

*Note that in the SAGE VST test the inertial coordinate system is oriented with the positive $X$ axis down, parallel to the acceleration of gravity. The value of $m g$ in Eq. (B.2) is the magnitude of the force along the $X$, axis, with the $Y$ and $Z$ components being zero. 


$$
\begin{gathered}
T_{21}^{B E F}=\cos R_{z} \sin R_{y} \sin R_{x}+\sin R_{z} \cos R_{x} \\
T_{22}^{B E F}=\cos R_{z} \cos R_{x}-\sin R_{z} \sin R_{y} \sin R_{x} \\
T_{23}^{B E F}=-\cos R_{y} \sin R_{x} \\
T_{31}^{B E F}=\sin R_{z} \sin R_{x}-\cos R_{z} \sin R_{y} \cos R_{x} \\
T_{32}^{B E F}=\sin R_{z} \sin R_{y} \cos R_{x}+\cos R_{z} \sin R_{x} \\
T_{33}^{B E F}=\cos R_{y} \cos R_{x}
\end{gathered}
$$

\section{B.5 Aerodynamic Forces and Moments}

The aerodynamic force and moment coefficients are required to estimate the aerodynamic parameter which they are a function of. The aerodynamic forces and moments are defined as shown in Eqs. (B.15) - B.20).

$$
\begin{aligned}
F_{A, x} & =q_{\infty} C_{X} S \\
F_{A, y} & =q_{\infty} C_{Y} S \\
F_{A, z} & =q_{\infty} C_{Z} S \\
M_{A, x} & =q_{\infty} C_{l} S d \\
M_{A, y} & =q_{\infty} C_{m} S d \\
M_{A, z} & =q_{\infty} C_{n} S d
\end{aligned}
$$

Using Eqs. (B.1) and (B.2) in conjunction with Eqs. (B.15) - (B.20), the force and moment coefficients are calculated.

$$
\begin{gathered}
C_{X}=-C_{A}=\frac{1}{q_{\infty} S}\left(F_{x}-F_{g, x}-F_{t r, x}\right) \\
C_{Y}=\frac{1}{q_{\infty} S}\left(F_{y}-F_{g, y}-F_{t r, y}\right) \\
C_{Z}=-C_{N}=\frac{1}{q_{\infty} S}\left(F_{z}-F_{g, z}-F_{t r, z}\right) \\
C_{l}=\frac{1}{q_{\infty} S d}\left(M_{x}-M_{t r, x}\right) \\
C_{m}=\frac{1}{q_{\infty} S d}\left(M_{y}-M_{t r, y}\right) \\
C_{n}=\frac{1}{q_{\infty} S d}\left(M_{z}-M_{t r, z}\right)
\end{gathered}
$$




\section{B.6 Tether Forces and Moments}

The tether force and moment vectors are defined as:

$$
\begin{gathered}
\mathbf{F}_{t r}=F_{t r, x} \mathbf{i}+F_{t r, y} \mathbf{j}+F_{t r, z} \mathbf{k} \\
\mathbf{M}_{t r}=M_{t r, x} \mathbf{i}+M_{t r, y} \mathbf{j}+M_{t r, z} \mathbf{k}
\end{gathered}
$$

The tether only exerts a force at the tether attachment point and the moment is due to the offset of this point with the models center of mass. The geometry of the tether is presented in Figure B.1. The components of the tether force are:

$$
\left\{\begin{array}{l}
F_{t r, x} \\
F_{t r, y} \\
F_{t r, z}
\end{array}\right\}=\frac{F_{t r}}{\left|\mathbf{r}_{t r}\right|}\left\{\begin{array}{l}
x_{t r} \\
y_{t r} \\
z_{t r}
\end{array}\right\}
$$

The tether force acts along the vector between the attachment point and the centering ring, $\mathbf{r}_{t r}$, and has a magnitude, $F_{t r}$. The values of these terms are defined in Eqs. (B.32) and (B.33). The moment vector is the cross product of the force vector with the location of the tether attachment point relative to the center of mass, $\mathbf{r}_{t}$, as defined in the Eq. (B.35).

$$
\begin{gathered}
\mathbf{M}_{t r}=\mathbf{F}_{t r} \times \mathbf{r}_{t} \\
\left\{\begin{array}{l}
M_{t r, x} \\
M_{t r, y} \\
M_{t r, z}
\end{array}\right\}=\left\{\begin{array}{l}
y_{t} F_{t r, z}-z_{t} F_{t r, y} \\
z_{t} F_{t r, x}-x_{t} F_{t r, z} \\
x_{t} F_{t r, y}-y_{t} F_{t r, x}
\end{array}\right\}
\end{gathered}
$$

\section{B.6.1 Force Magnitude}

The model for the tether force magnitude was determined from experimental measurements (see Chapter 2). The model is a linear fit of the tether force versus the dynamic pressure.

$$
F_{t r}=q_{\infty}\left(C_{D} S\right)_{t r}-W_{t r}
$$

Table B.1: Tether force model parameters

\begin{tabular}{lcl}
\hline Parameter & Value & Units \\
\hline$\left(C_{D} S\right)_{t r}$ & $0.0140(0.0013)$ & $\mathrm{ft}^{2}\left(\mathrm{~m}^{2}\right)$ \\
$W_{t r}$ & $0.0037(0.0037)$ & $\mathrm{lb}(\mathrm{N})$ \\
\hline
\end{tabular}

The tether force model parameters in Table B.1 are for the specific test described by this thesis and should be applied with caution to data collected in other wind tunnel tests. Chapter 2 describes the testing method so it may be repeated. 


\section{B.6.2 Tether Geometry}

The tether force is directed toward the centering ring at all times. This vector is defined as shown in Eq. B.33.

$$
\mathbf{r}_{t r}=x_{t r} \mathbf{i}+y_{t r} \mathbf{j}+z_{t r} \mathbf{k}
$$

The components can be determined with Eq. (B.34) from the ring position, model position and tether attachment point (or vectorally: $\mathbf{r}_{r}, \mathbf{r}_{o}$, and $\mathbf{r}_{t, o}$ respectively) as defined in Figure B.1.

$$
\left\{\begin{array}{l}
x_{t r} \\
y_{t r} \\
z_{t r}
\end{array}\right\}=\left[T^{B E F}\right]^{T}\left\{\begin{array}{c}
X_{r}-X \\
Y_{r}-Y \\
Z_{r}-Z
\end{array}\right\}-\left\{\begin{array}{l}
x_{t, o} \\
y_{t, o} \\
z_{t, o}
\end{array}\right\}
$$

The location of the tether attachment point is defined as shown in Eq. (B.35).

$$
\mathbf{r}_{t}=x_{t} \mathbf{i}+y_{t} \mathbf{j}+z_{t} \mathbf{k}
$$

The components are determined with Eq. B.36 from the locations of the tether attachment point and the center of mass with regard to the origin (or vectorialy: $\mathbf{r}_{t, o}$ and $\mathbf{r}_{c m}$ respectively) also defined in Figure B.1.

$$
\left\{\begin{array}{l}
x_{t} \\
y_{t} \\
z_{t}
\end{array}\right\}=\left\{\begin{array}{l}
x_{t, o} \\
y_{t, o} \\
z_{t, o}
\end{array}\right\}-\left\{\begin{array}{l}
x_{c m} \\
y_{c m} \\
z_{c m}
\end{array}\right\}
$$

\section{B.7 Auxiliary Equations}

The following are the auxiliary equations required for the PID code. These variables are used both in the above force calculations and as regressors for the aerodynamic coefficients.

\section{B.7.1 Airspeed and Wind Components}

$$
\begin{gathered}
\left\{\begin{array}{c}
u_{\infty} \\
v_{\infty} \\
w_{\infty}
\end{array}\right\}=\left\{\begin{array}{c}
u \\
v \\
w
\end{array}\right\}-\left\{\begin{array}{c}
u_{W} \\
v_{W} \\
w_{W}
\end{array}\right\} \\
\left\{\begin{array}{c}
u_{W} \\
v_{W} \\
w_{W}
\end{array}\right\}=\left[T^{B E F}\right]^{T}\left\{\begin{array}{c}
-V_{W} \\
0 \\
0
\end{array}\right\}
\end{gathered}
$$




$$
\begin{aligned}
& V_{\infty}=\sqrt{u_{\infty}^{2}+v_{\infty}^{2}+w_{\infty}^{2}} \\
& \left\{\begin{array}{c}
\dot{u}_{\infty} \\
\dot{v}_{\infty} \\
\dot{w}_{\infty}
\end{array}\right\}=\left\{\begin{array}{c}
\dot{u} \\
\dot{v} \\
\dot{w}
\end{array}\right\}-\left\{\begin{array}{c}
\dot{u}_{W} \\
\dot{v}_{W} \\
\dot{w}_{W}
\end{array}\right\} \\
& \left\{\begin{array}{c}
\dot{u}_{W} \\
\dot{v}_{W} \\
\dot{w}_{W}
\end{array}\right\}=\left[T^{B E F}\right]^{T}\left\{\begin{array}{c}
-\dot{V}_{W} \\
0 \\
0
\end{array}\right\}+\left[\dot{T}^{B E F}\right]^{T}\left\{\begin{array}{c}
-V_{W} \\
0 \\
0
\end{array}\right\} \\
& {\left[\dot{T}^{B E F}\right]=\left[\begin{array}{lll}
\dot{T}_{11}^{B E F} & \dot{T}_{12}^{B E F} & \dot{T}_{13}^{B E F} \\
\dot{T}_{21}^{B E F} & \dot{T}_{22}^{B E F} & \dot{T}_{23}^{B E F} \\
\dot{T}_{31}^{B E F} & \dot{T}_{32}^{B E F} & \dot{T}_{33}^{B E F}
\end{array}\right]} \\
& \dot{T}_{11}^{B E F}=-\dot{R}_{z} \sin R_{z} \cos R_{y}-\dot{R}_{y} \cos R_{z} \sin R_{y} \\
& \dot{T}_{12}^{B E F}=-\dot{R}_{z} \cos R_{z} \cos R_{y}+\dot{R}_{y} \sin R_{z} \sin R_{y} \\
& \dot{T}_{13}^{B E F}=\dot{R}_{y} \cos R_{y} \\
& \dot{T}_{21}^{B E F}=\dot{R}_{z}\left(\cos R_{z} \cos R_{x}-\sin R_{z} \sin R_{y} \sin R_{x}\right) \\
& +\dot{R}_{y}\left(\cos R_{z} \cos R_{y} \sin R_{x}\right) \\
& +\dot{R}_{x}\left(\cos R_{z} \sin R_{y} \cos R_{x}-\sin R_{z} \sin R_{x}\right) \\
& \dot{T}_{22}^{B E F}=-\dot{R}_{z}\left(\cos R_{z} \sin R_{y} \sin R_{x}+\sin R_{z} \cos R_{x}\right) \\
& -\dot{R}_{y}\left(\sin R_{z} \cos R_{y} \sin R_{x}\right) \\
& -\dot{R}_{x}\left(\sin R_{z} \sin R_{y} \cos R_{x}+\cos R_{z} \sin R_{x}\right) \\
& \dot{T}_{23}^{B E F}=\dot{R}_{y} \sin R_{y} \sin R_{x}-\dot{R}_{x} \cos R_{y} \cos R_{x} \\
& \dot{T}_{31}^{B E F}=\dot{R}_{z}\left(\sin R_{z} \sin R_{y} \cos R_{x}+\cos R_{z} \sin R_{x}\right) \\
& -\dot{R}_{y}\left(\cos R_{z} \cos R_{y} \cos R_{x}\right) \\
& +\dot{R}_{x}\left(\cos R_{z} \sin R_{y} \sin R_{x}+\sin R_{z} \cos R_{x}\right) \\
& \dot{T}_{32}^{B E F}=\dot{R}_{z}\left(\cos R_{z} \sin R_{y} \cos R_{x}-\sin R_{z} \sin R_{x}\right) \\
& +\dot{R}_{y}\left(\sin R_{z} \cos R_{y} \cos R_{x}\right) \\
& -\dot{R}_{x}\left(\sin R_{z} \sin R_{y} \sin R_{x}-\cos R_{z} \cos R_{x}\right) \\
& \dot{T}_{33}^{B E F}=-\dot{R}_{y} \sin R_{y} \cos R_{x}-\dot{R}_{x} \cos R_{y} \sin R_{x}
\end{aligned}
$$

${ }^{\dagger}$ Note that in the SAGE VST test the inertial coordinate system is oriented with the positive $X$ axis down anti-parallel to the wind velocity flow field. The flow field is assumed to uniform over the area of the test section, thus the value of $V_{W}$, in Eq. (B.37), is the magnitude of the wind to reflect this. The $Y$ and $Z$ wind components are assumed to be zero. 


\section{B.7.2 Dynamic Pressure and Wind Angles}

$$
\begin{aligned}
& q_{\infty}=\frac{1}{2} \rho_{\infty} V_{\infty}^{2} \\
& \alpha=\arctan \left(\frac{w_{\infty}}{u_{\infty}}\right) \quad-\pi<\alpha \leq \pi \\
& \beta=\arcsin \left(\frac{v_{\infty}}{V_{\infty}}\right) \quad-\frac{\pi}{2} \leq \beta \leq \frac{\pi}{2} \\
& \alpha_{T}=\arccos \left(\frac{u_{\infty}}{V_{\infty}}\right) \quad 0 \leq \alpha_{T} \leq \pi \\
& \phi_{\alpha_{T}}=\arctan \left(\frac{v_{\infty}}{w_{\infty}}\right) \quad-\pi<\phi_{\alpha_{T}} \leq \pi \\
& \dot{\alpha}=\frac{u_{\infty} \dot{w}_{\infty}-\dot{u}_{\infty} w_{\infty}}{u_{\infty}^{2}+w_{\infty}^{2}} \\
& \dot{\beta}=\frac{\dot{v}_{\infty} V_{\infty}^{2}-v_{\infty}\left(u_{\infty} \dot{u}_{\infty}+v_{\infty} \dot{v}_{\infty}+w_{\infty} \dot{w}_{\infty}\right)}{V_{\infty}^{2} \sqrt{u_{\infty}^{2}+w_{\infty}^{2}}} \\
& \dot{\alpha}_{T}=\frac{u_{\infty}\left(u_{\infty} \dot{u}_{\infty}+v_{\infty} \dot{v}_{\infty}+w_{\infty} \dot{w}_{\infty}\right)-\dot{u}_{\infty} V_{\infty}^{2}}{V_{\infty}^{2} \sqrt{v_{\infty}^{2}+w_{\infty}^{2}}} \\
& \dot{\phi}_{\alpha_{T}}=\frac{\dot{v}_{\infty} w_{\infty}-v_{\infty} \dot{w}_{\infty}}{v_{\infty}^{2}+w_{\infty}^{2}} \\
& \alpha=\arctan \left(\frac{\sin \alpha_{T} \cos \phi_{\alpha_{T}}}{\cos \alpha_{T}}\right) \quad-\pi<\alpha \leq \pi \\
& \beta=\arcsin \left(\sin \alpha_{T} \sin \phi_{\alpha_{T}}\right) \quad-\frac{\pi}{2} \leq \beta \leq \frac{\pi}{2} \\
& \alpha_{T}=\arccos (\cos \alpha \cos \beta) \quad 0 \leq \alpha_{T} \leq \pi \\
& \phi_{\alpha_{T}}=\arctan \left(\frac{\sin \beta}{\sin \alpha \cos \beta}\right) \quad-\pi<\phi_{\alpha_{T}} \leq \pi
\end{aligned}
$$

\section{B.7.3 Additional Equations}

$$
\begin{gathered}
h=-X \\
M_{\infty}=\frac{V_{\infty}}{a_{\infty}} \\
R e_{\infty}=\frac{\rho_{\infty} V_{\infty} d}{\mu_{\infty}}
\end{gathered}
$$




\section{B.8 Shifting the Static Aerodynamic Moment Coefficients}

The aerodynamic forces and moments at the lander coordinate system origin are given in Eqs. (B.60) and (B.61) respectively.

$$
\begin{aligned}
\mathbf{F}_{A} & =\left\{\begin{array}{l}
F_{A, x} \\
F_{A, y} \\
F_{A, z}
\end{array}\right\}=q_{\infty} S\left\{\begin{array}{c}
-C_{A} \\
C_{Y} \\
-C_{N}
\end{array}\right\} \\
\mathbf{M}_{A, o} & =\left\{\begin{array}{l}
M_{A, x_{o}} \\
M_{A, y_{o}} \\
M_{A, z_{o}}
\end{array}\right\}=q_{\infty} S d\left\{\begin{array}{l}
C_{l_{o}} \\
C_{m_{o}} \\
C_{n_{o}}
\end{array}\right\}
\end{aligned}
$$

The center of mass of the lander is offset from the lander geometric coordinate system origin. At the center of mass the aerodynamic force remains the same but the aerodynamic moment will have a contribution from the force. The moment arm that is defined as the location of the center of mass relative to the lander coordinate system origin, $\left(\mathbf{r}_{c m}=x_{c m} \mathbf{i}+\right.$ $y_{c m} \mathbf{j}+z_{c m} \mathbf{k}$ ). The aerodynamic moment at the center of mass is given in Eqs. (B.62) and (B.63).

$$
\begin{gathered}
\mathbf{M}_{A}=\mathbf{M}_{A, o}+\mathbf{F}_{A} \times \mathbf{r}_{c m} \\
q_{\infty} S d\left\{\begin{array}{c}
C_{l} \\
C_{m} \\
C_{n}
\end{array}\right\}=q_{\infty} S d\left\{\begin{array}{l}
C_{l_{o}} \\
C_{m_{o}} \\
C_{n_{o}}
\end{array}\right\}+q_{\infty} S\left\{\begin{array}{c}
C_{N} y_{c m}+C_{Y} z_{c m} \\
-C_{N} x_{c m}+C_{A} z_{c m} \\
-C_{Y} x_{c m}-C_{A} y_{c m}
\end{array}\right\}
\end{gathered}
$$

By dividing through by $q_{\infty} S d$ gives the final form of the aerodynamic moment coefficients at the center of mass.

$$
\begin{gathered}
C_{l}=C_{l_{o}}+C_{N} \frac{y_{c m}}{d}+C_{Y} \frac{z_{c m}}{d} \\
C_{m}=C_{m_{o}}-C_{N} \frac{x_{c m}}{d}+C_{A} \frac{z_{c m}}{d} \\
C_{n}=C_{n_{o}}-C_{Y} \frac{x_{c m}}{d}-C_{A} \frac{y_{c m}}{d}
\end{gathered}
$$

\section{B.9 Transformation of Coordinate Systems and Euler Angles}

The data collected in the VST is done so with a modified inertial coordinate system and nonstandard Euler angles, $\left(R_{x}, R_{y}, R_{z}\right)$. This is done to avoid the singularities associated 
with the standard aerospace Euler angles, $(\psi, \theta, \phi)$. The spin tunnel data also uses a modified Euler angle sequence $(X, Y, Z$ rotation sequence) as opposed to a conventional aircraft $(Z$, $Y, X$ rotation sequence). Simulations, operating on the conventional coordinate system and Euler angles, were used to verify PID code. The following is the equations required for the conversion between the spin tunnel coordinates and the conventional coordinate system used in simulations.

$$
\begin{gathered}
X_{\text {sim }}=Y \\
Y_{\text {sim }}=Z \\
Z_{\text {sim }}=X \\
\psi=\arctan \left(\frac{\sin R_{z} \sin R_{x}-\cos R_{z} \sin R_{y} \cos R_{x}}{\cos R_{z} \sin R_{y} \sin R_{x}+\sin R_{z} \cos R_{x}}\right) \quad-\pi<\psi \leq \pi \\
\theta=\arcsin \left(-\cos R_{z} \cos R_{y}\right) \quad \frac{-\pi}{2} \leq \theta \leq \frac{\pi}{2} \\
\phi=\arctan \left(\frac{-\sin R_{z} \cos R_{y}}{\sin R_{y}}\right) \quad-\pi<\phi \leq \pi \\
R_{x}=\arctan \left(\frac{-\cos \phi \sin \theta \cos \psi-\sin \phi \sin \psi}{\cos \phi \sin \theta \sin \psi-\sin \phi \cos \psi}\right) \quad-\pi<R_{x} \leq \pi \\
R_{y}=\arcsin (\cos \phi \cos \theta) \quad-\pi \\
R_{z}=\arctan \left(\frac{\sin \phi \cos \theta}{\sin \theta}\right) \quad-\pi<R_{y} \leq \frac{\pi}{2}
\end{gathered}
$$




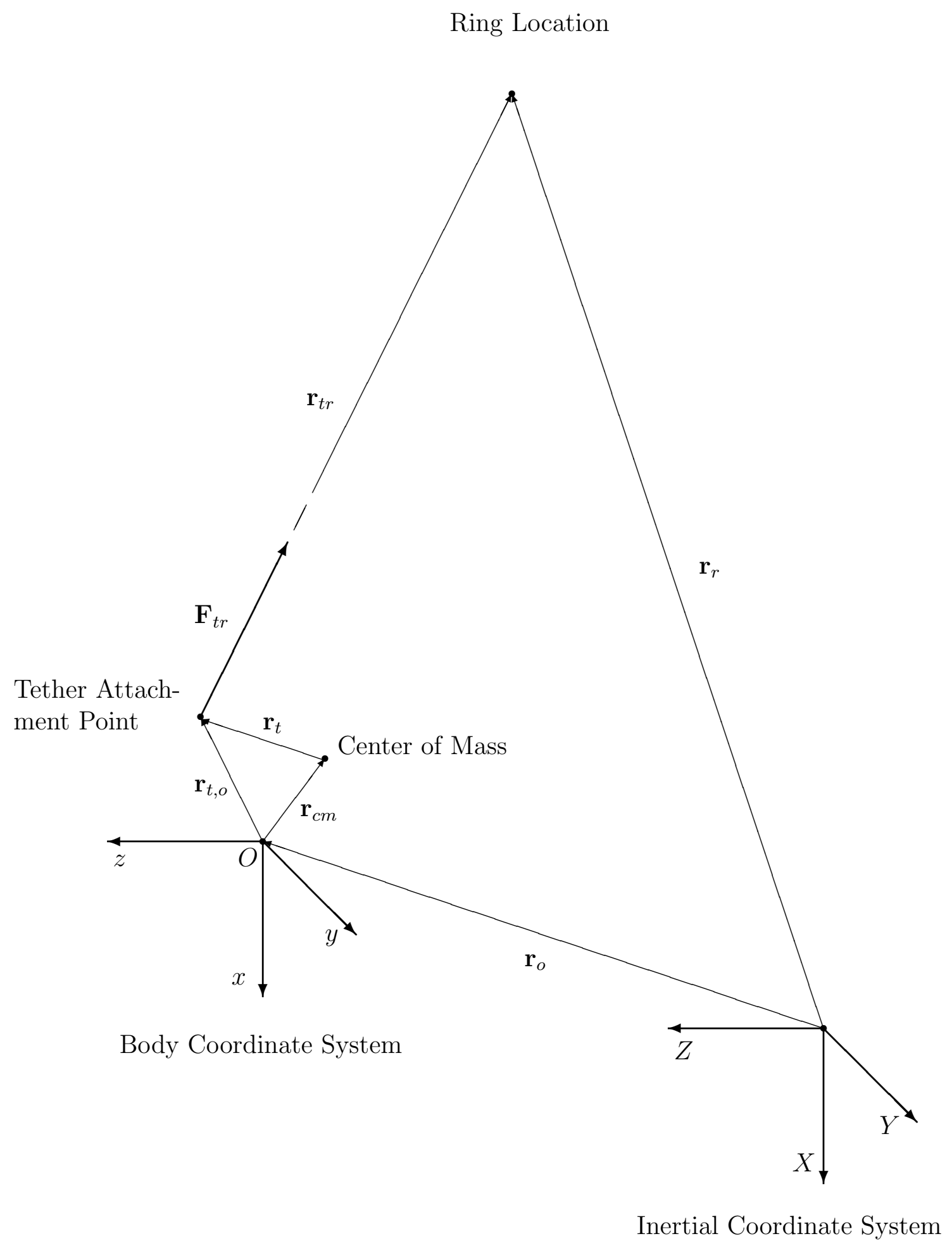

Figure B.1: Tether geometry within VST. 


\section{Appendix C: Code Verification}

This appendix presents simulated data, with the aerodynamic model identified in Chapter 5 , which is entered into the PID code to compare the results to that of the simulation. The simulation has been independently verified.

\section{C.1 Simulated Data}

Figure C.1 shows the simulated data that was entered into the PID code. The signal was assumed to be perfect and thus was not smoothed. A derivative function included in the SIDPAC software of reference [4] in order to obtain the necessary derivatives of the data. The simulation used all the mass and geometric properties of the SAGE lander described in Chapter 2. The simulated run is similar to a perturbed run in that the SAGE lander model is given an initial total angle of attack of $\alpha_{T}=25^{\circ}$. The oscillations die out over the course of the simulated run.

\section{C.2 Equations of Motion}

Figures C.2 and C.3 show the comparisons of the PID code results to the related simulation results. The most important values to the PID analysis are those that are used for the force and moment coefficients and the regressors. Many other values are obtained for comparison, all of which match up in a similar manner as those in the figures. The equations of motion subroutine used in the PID code is capable of calculating these with little error.

\section{C.3 Parameter Identification of Simulated Data}

The estimates of the aerodynamic parameters are given in Table C.1 and are compared to the values used in the simulation (these are the selected parameters also presented in Table 5.5). The aerodynamic parameters are estimated with high accuracy. The least accurate aerodynamic parameters are the squared and cubed parameters. Using low angle simulated data the PID code has similar issues seen in the unperturbed data set analysis where the cubed terms were greatly overestimated. This means that the best results are achieved from the perturbed and combined data sets. 
Table C.1: Results of PID analysis on simulated data.

\begin{tabular}{llccl}
\hline Coefficient & Parameter & PID Estimate & Simulation Actual & \% Error \\
\hline$C_{A}$ & $C_{A_{T}}$ & 6.6622 & 6.6620 & 0.00 \\
\hline \multirow{2}{*}{$C_{N}$} & $C_{N_{\sin \alpha_{T}}}$ & 3.4740 & 3.5129 & 1.11 \\
& $C_{N_{\sin \alpha_{T}}}$ & -2.1405 & -2.0035 & 6.84 \\
\hline \multirow{2}{*}{$C_{l_{o}}$} & $C_{l_{o, T}}$ & 0.0118 & 0.0125 & 5.60 \\
& $C_{l_{p}}$ & -5.3758 & -5.6816 & 5.38 \\
\hline \multirow{4}{*}{$C_{m_{o}}$} & $C_{m_{\sin \alpha_{T}}}$ & -2.0706 & -2.0655 & 0.25 \\
& $C_{m_{\sin \alpha_{T}}}$ & 2.5067 & 2.5278 & 0.83 \\
& $C_{m_{1}}$ & -3.3947 & -3.3025 & 2.79 \\
& $C_{m_{2}}$ & -200.7541 & -205.4289 & 2.28 \\
\hline \multirow{4}{*}{$C_{n_{o}}$} & $C_{m_{\sin \alpha_{T}}}$ & -2.0688 & -2.0655 & 0.16 \\
& $C_{m_{\sin 3} \alpha_{T}}$ & 2.4105 & 2.5278 & 4.64 \\
& $C_{n_{1}}$ & -3.3710 & -3.3025 & 2.07 \\
& $C_{n_{2}}$ & -202.3803 & -205.4289 & 1.48 \\
\hline
\end{tabular}




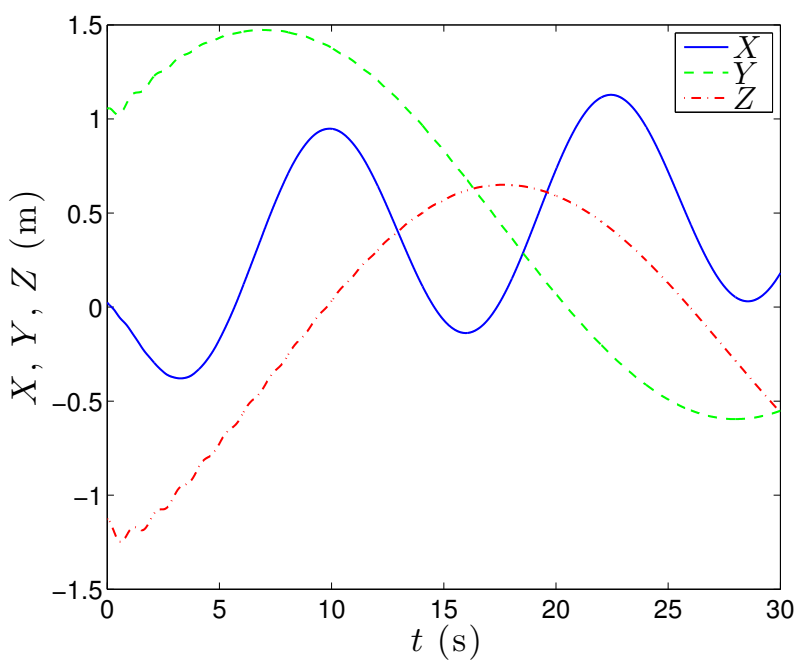

(a) $X, Y$, and $Z$ simulated data.

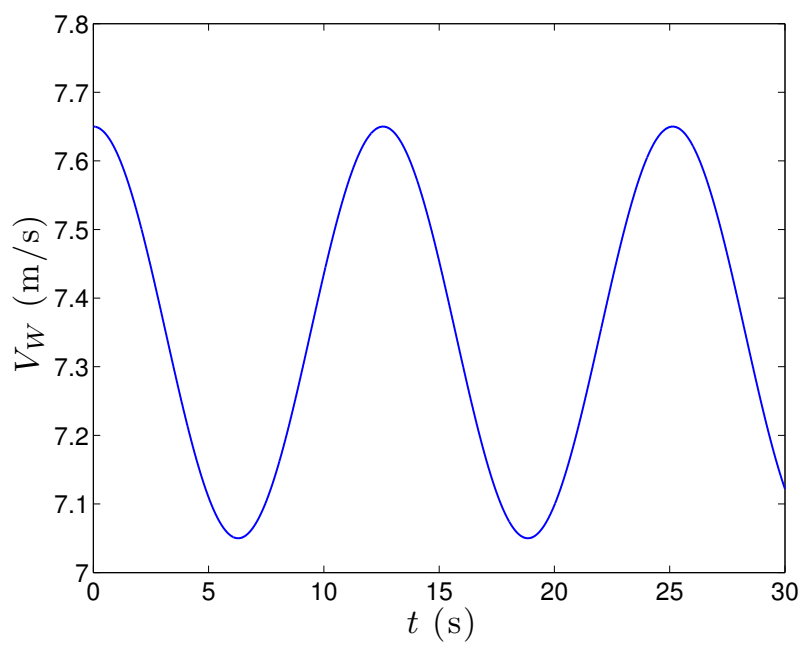

(c) $V_{W}$ simulated data.

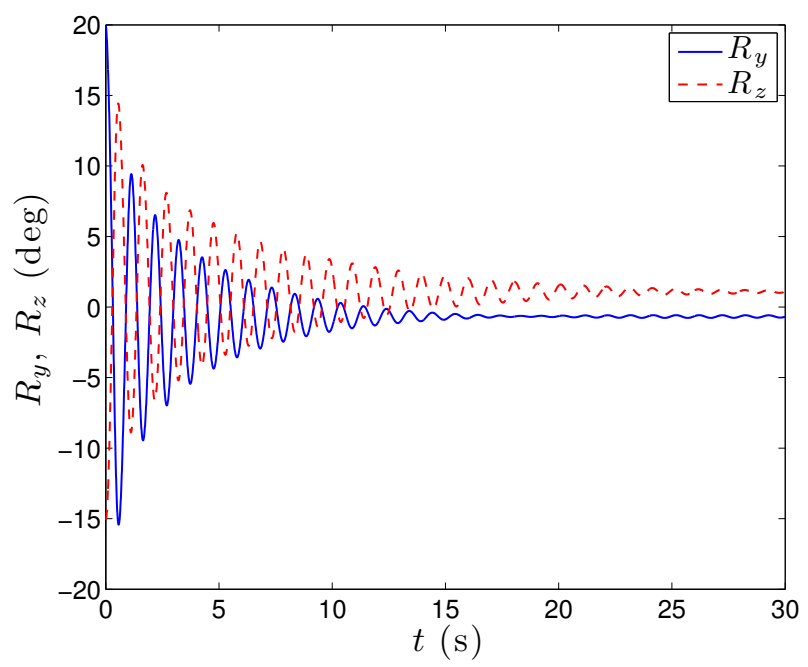

(b) $R_{y}$ and $R_{z}$ simulated data.

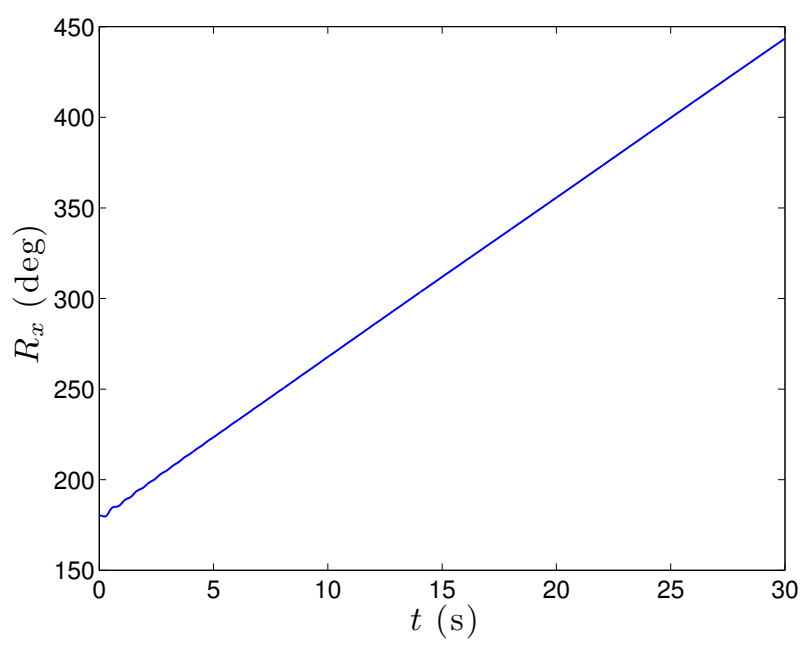

(d) $R_{x}$ simulated data.

Figure C.1: Simulated data for validation of the PID code. 


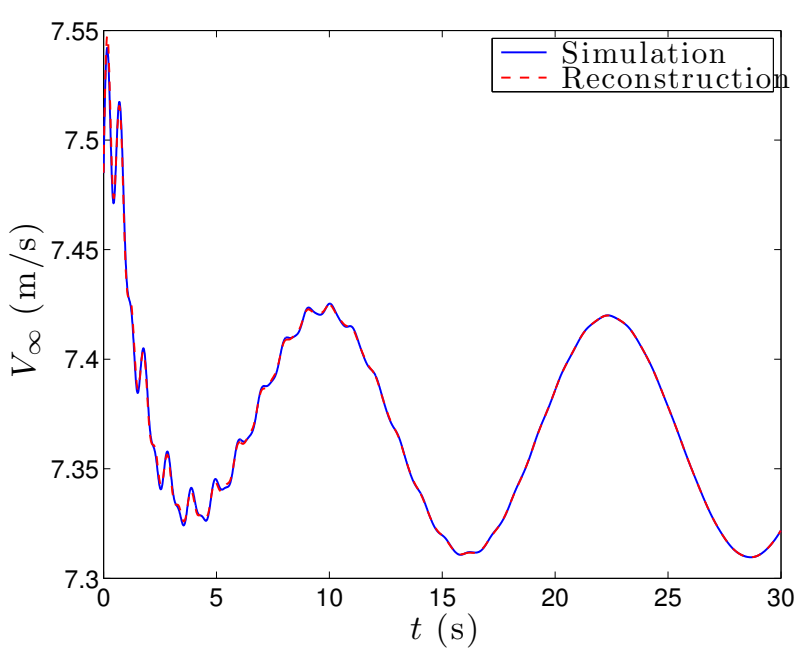

(a) $V_{\infty}$ comparison.

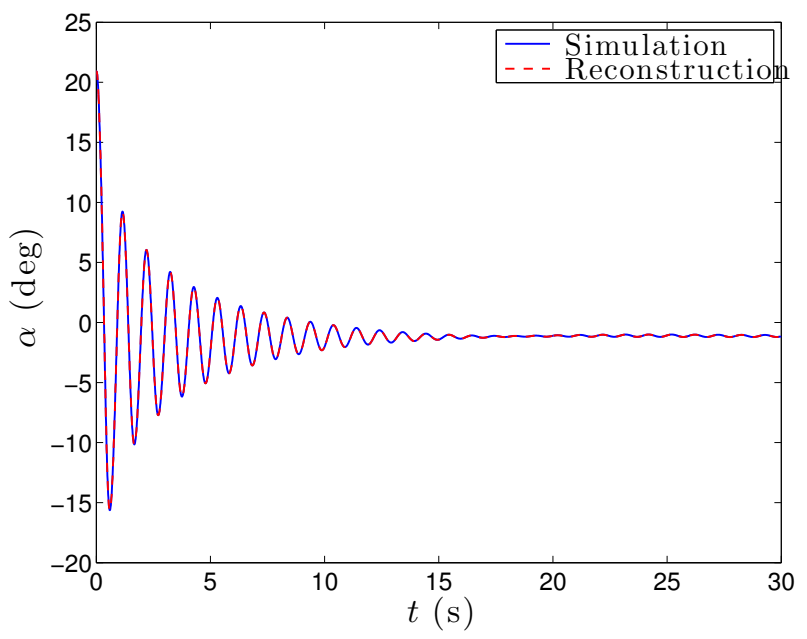

(c) $\alpha$ comparison.

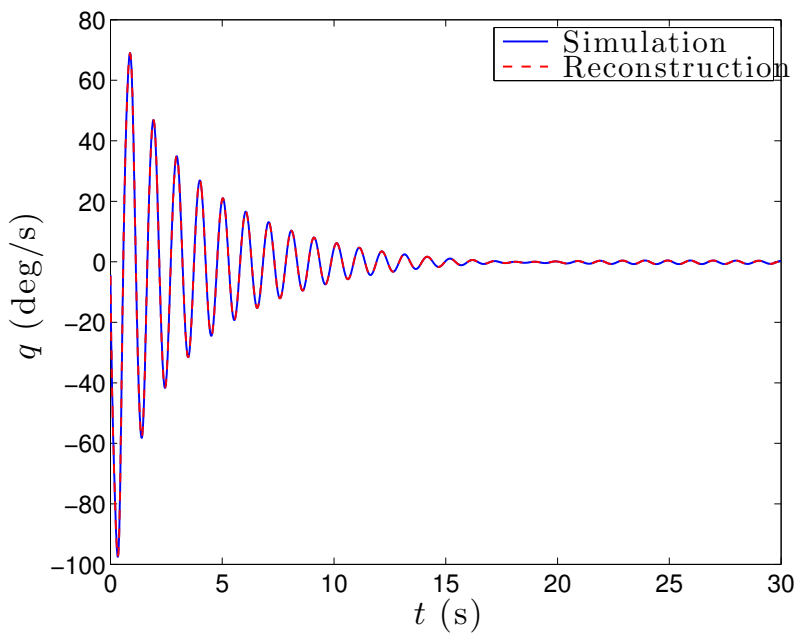

(e) $q$ comparison.

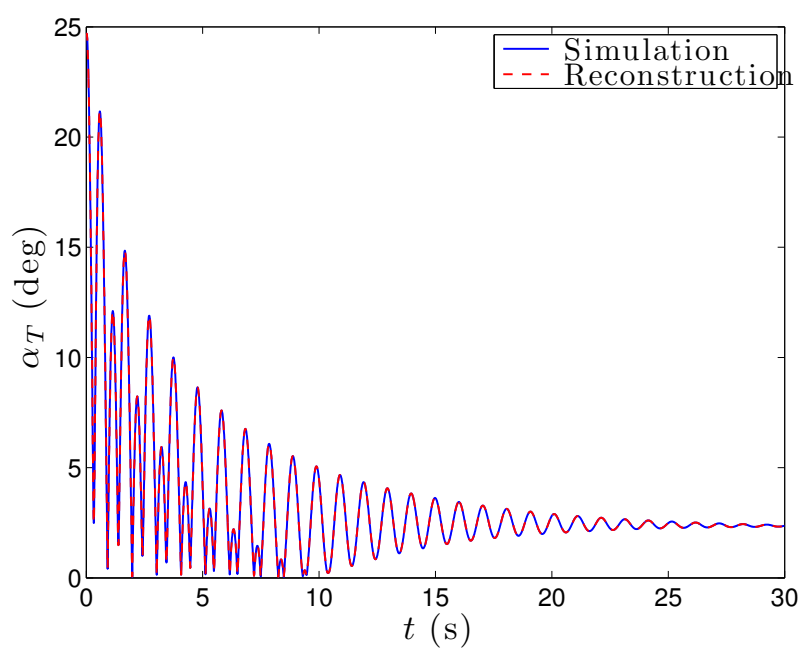

(b) $\alpha_{T}$ comparison.

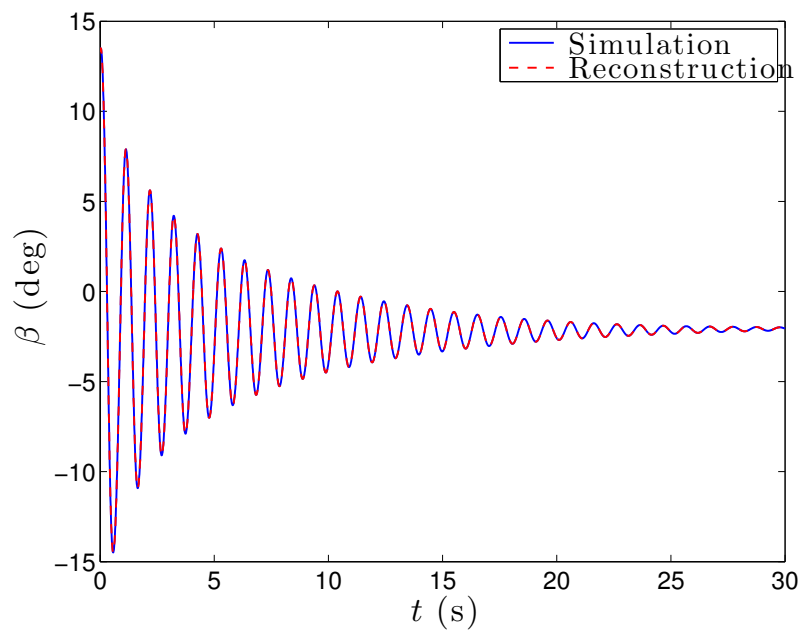

(d) $\beta$ comparison.

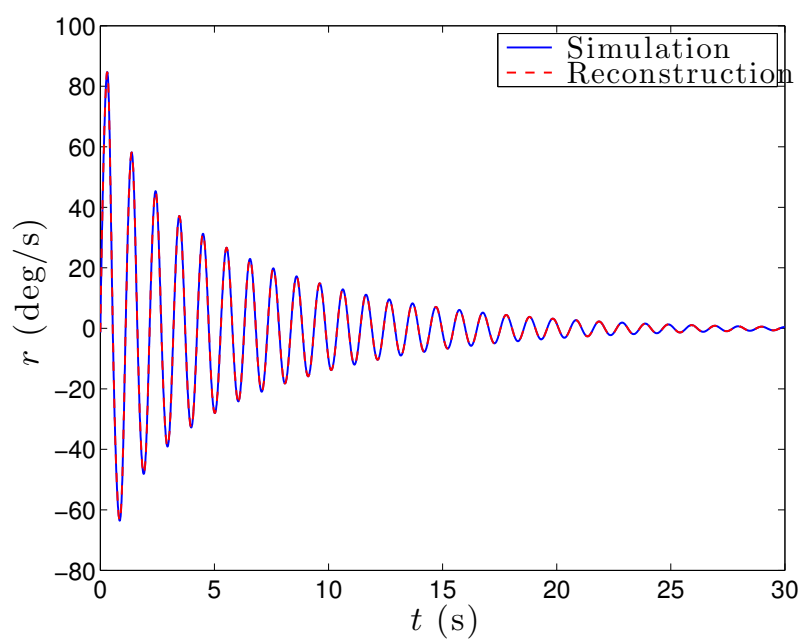

(f) $r$ comparison.

Figure C.2: Comparison of simulated and reconstructed dynamic values used in the PID code. 


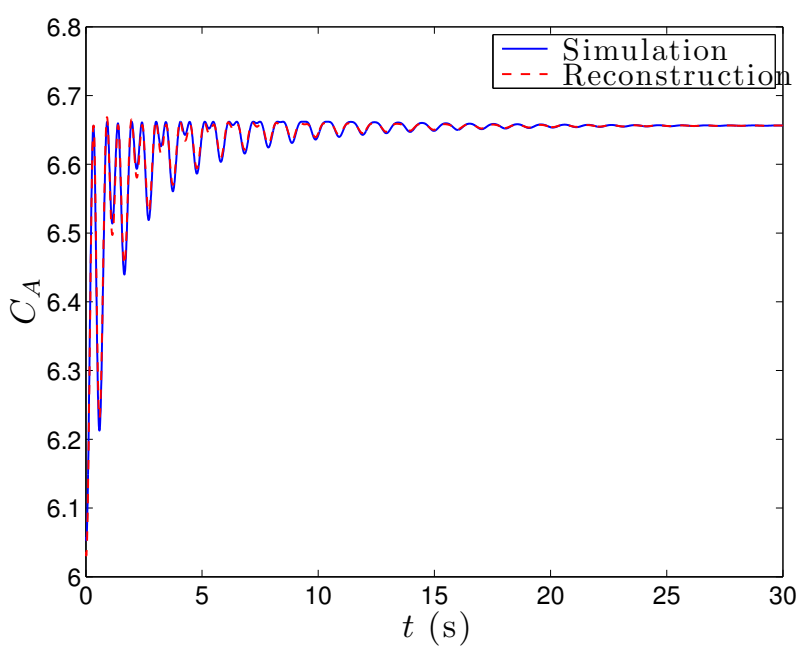

(a) $C_{A}$ comparison.

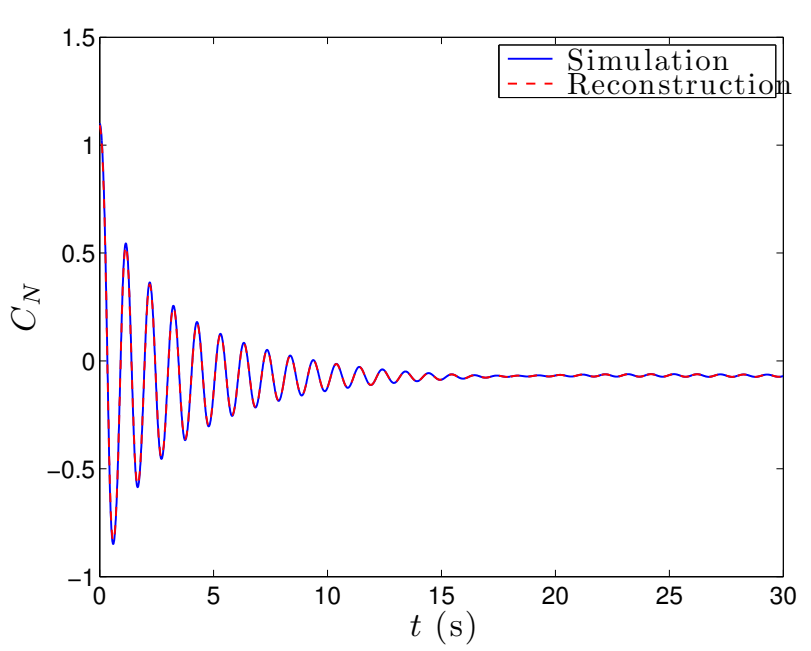

(c) $C_{N}$ comparison.

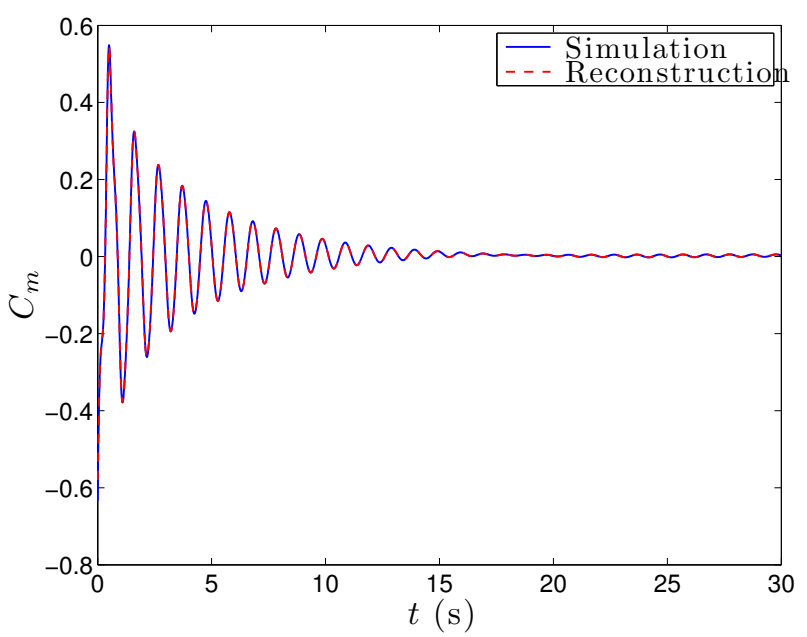

(e) $C_{m}$ comparison.

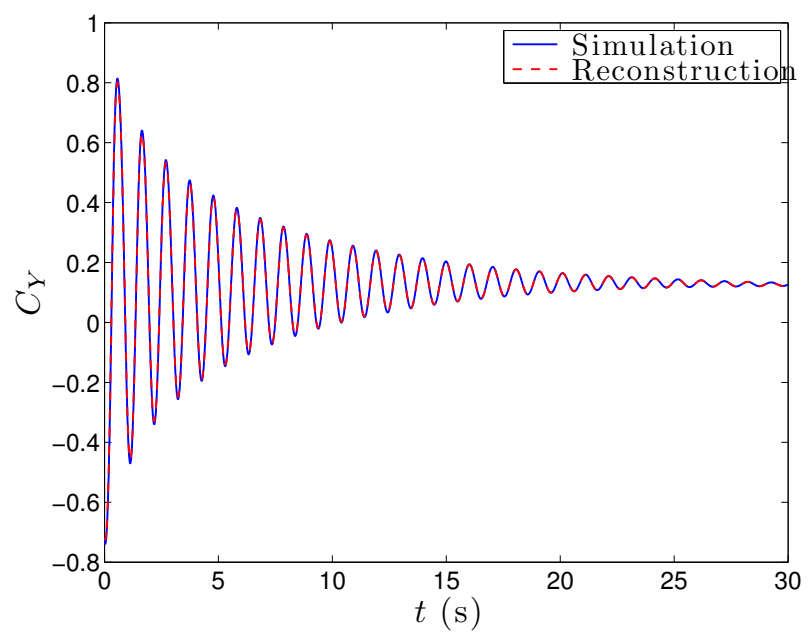

(b) $C_{Y}$ comparison.

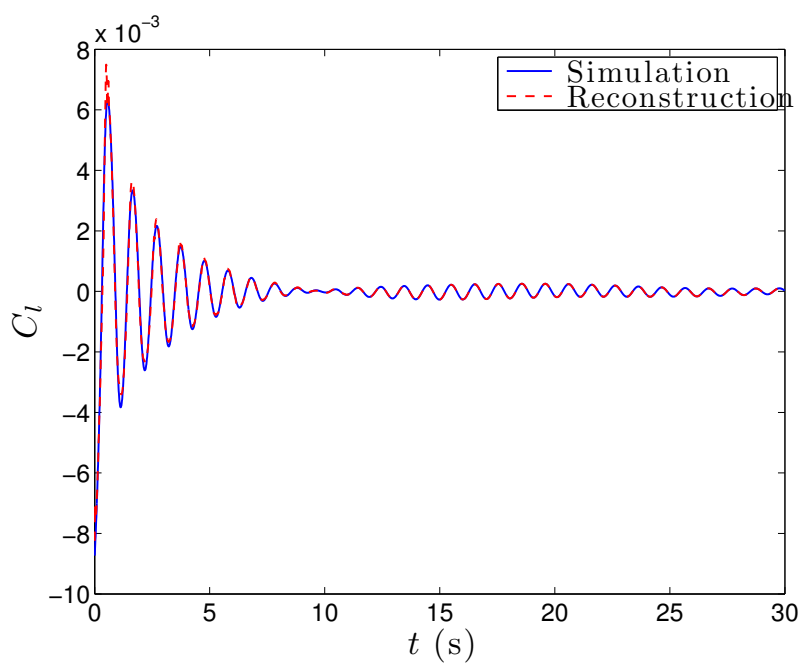

(d) $C_{l}$ comparison.

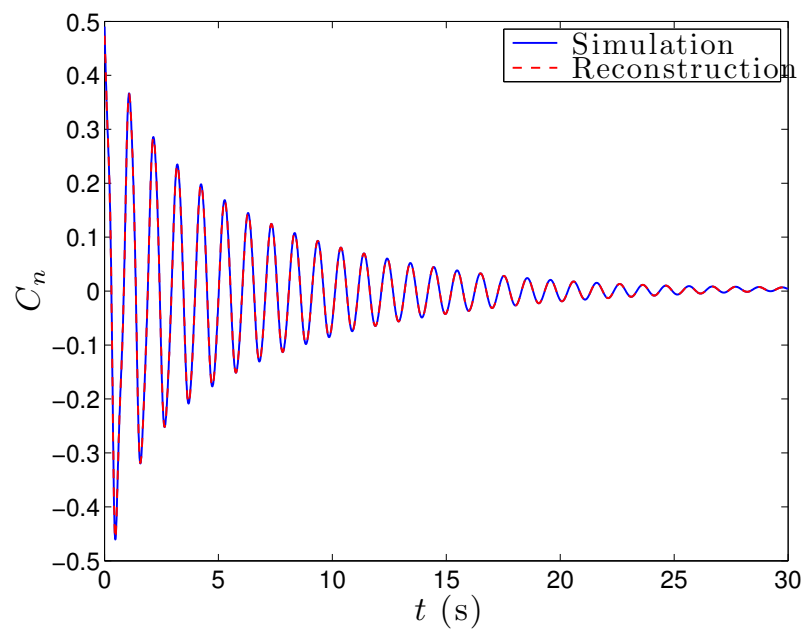

(f) $C_{n}$ comparison.

Figure C.3: Comparison of simulated and reconstructed force and moment coefficients used in the PID code. 